\title{
Nuclear Reactors Built, Being Built, or Planned: 1991
}

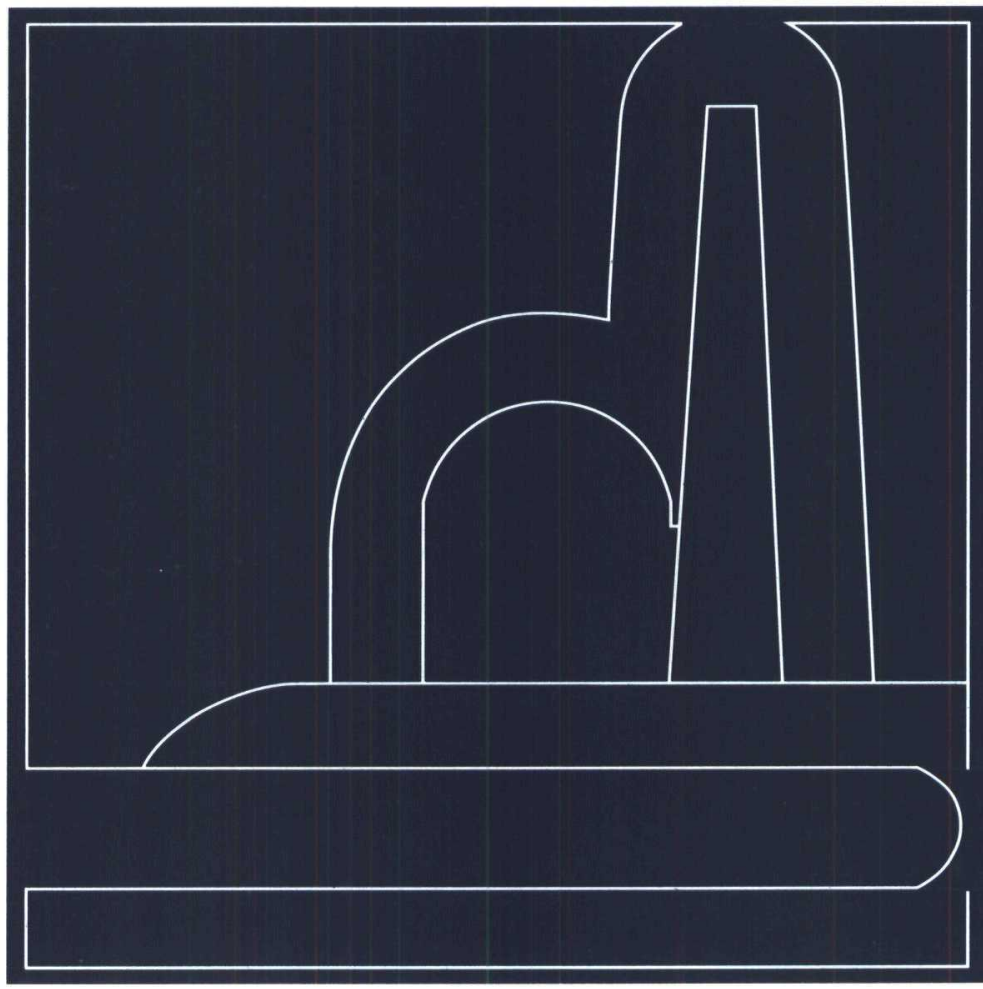

Prepared for:

U.S. DEPARTMENT OF ENERGY

Assistant Secretary for Nuclear Energy

Prepared by:

Office of Scientific and

Technical Information 


\section{DISCLAIMER}

This report was prepared as an account of work sponsored by an agency of the United States Government. Neither the United States Government nor any agency Thereof, nor any of their employees, makes any warranty, express or implied, or assumes any legal liability or responsibility for the accuracy, completeness, or usefulness of any information, apparatus, product, or process disclosed, or represents that its use would not infringe privately owned rights. Reference herein to any specific commercial product, process, or service by trade name, trademark, manufacturer, or otherwise does not necessarily constitute or imply its endorsement, recommendation, or favoring by the United States Government or any agency thereof. The views and opinions of authors expressed herein do not necessarily state or reflect those of the United States Government or any agency thereof. 


\section{DISCLAIMER}

Portions of this document may be illegible in electronic image products. Images are produced from the best available original document. 
Available to DOE and DOE contractors from the Office of Scientific and Technical Information, P.O. Box 62, Oak Ridge, Tennessee 37831; prices available from (615) 576-8401.

Available to the public from the National Technical Information Service, U.S. Department of Commerce, 5285 Port Royal Rd., Springfield, Virginia 22161; prices available from (703) 487-4650. 
DOE/OSTI--8200-R55

\section{Nuclear Reactors Built, Being Built, or Planned 1991}

Prepared for:

\section{U.S. DEPARTMENT OF ENERGY}

Assistant Secretary for Nuclear Energy

Washington, D.C. 20585 
Blank Page 


\section{Preface}

Nuclear Reactors Built, Being Built, or Planned contains unclassified information about facilities built, being built, or planned in the United States for domestic use or export as of December 31, 1991. The Office of Scientific and Technical Information, U.S. Department of Energy, gathers this information annually from Washington headquarters and field offices of DOE, from the U.S. Nuclear Regulatory Commission, from the U.S. reactor manufacturers who are the principal nuclear contractors for foreign reactor locations, from U.S. and foreign embassies, and from foreign governmental nuclear departments.

The book is divided into three major sections: Section 1 consists of a reactor locator map and reactor tables; Section 2 includes nuclear reactors that are operating, being built, or planned; and Section 3 includes reactors that have been shut down permanently or dismantled.

Sections 2 and 3 contain the following classification of reactors: Civilian, Production, Military, Export, and Critical Assembly. Export reactor refers to a reactor for which the principal nuclear contractor is an American companyworking either independently or in cooperation with a foreign company (Part IV, in each Section). Critical assembly refers to an assembly of fuel and moderator that requires an external source of neutrons to initiate and maintain fission. A critical assembly is used for experimental measurements (Part V).

The various classes of reactors within these categories are defined as follows:

Central-Station Electric Power Plant: A nuclear power facility designed and constructed for operation on a utility system (Part I, section 1A).

Dual-Purpose Plant: A nuclear power facility designed, constructed, and operated for more than one primary purpose, for example, the production of nuclear materials and the generation of electricity or the use of reactor thermal energy for electrical generation and process-heat applications including desalting (Part I, section 1B).

ExperImental Power Reactor: A facility designed, engineered, constructed, and operated to test the technical feasibility of a concept or to provide the technical basis for a similar type nuclear power plant in a larger size. Design flexibility permits changes to prove various aspects of reactor technology including fuel, components, and configurations. Power-conversion equipment may or may not be included as part of the facility (Part I, section 2).

General Irradiation Test Reactor: A reactor having (1) a thermal power level exceeding $10 \mathrm{MW}$; (2) test loops or experimental facilities within, or in proximity to, the core; and (3) the use of nuclear radiation for testing the life or performance of reactor components as its major function (Part I, section 3A, and Part IV, section 2A).

High-Power Research and Teat Reactor: A reactor having a relatively high thermal power level (5 MW or greater) but not classed as a general irradiation test reactor (Part I, section 3B).

Safety-Research and Test Reactor: A reactor associated with a nuclear safety research or engineering-scale test program conducted for the purpose of developing basic design information or demonstrating safety characteristics of terrestrial and aerospace nuclear reactor systems (Part I, section 3C).

Research Reactor: A reactor-excluding that located at a university-whose nuclear radiations are used primarily as a research tool for basic or applied research and whose thermal power level is $10 \mathrm{MW}$ or less. It may include facilities for testing reactor materials (Part I, section 3D; Part III, section $3 B$; and Part IV, section 2B).

University Research and Teaching Reactor: A reactor located at a university and usually operated for the primary purpose of training in the operation and utilization of reactors and for instruction in reactor theory and performance (Part I, section 3E, and Part IV, section 2C).

Reactors are listed as operable under the following circumstances:

1. Reactors regulated by the NRC

-when an operating license is issued.

-when a reactor is temporarily shutdown because of technical reasons, modifications, or refueling.

2. Federal Government reactors

- when criticality is achieved.

- when a reactor is temporarily shutdown for safety improvements.

3. Reactors for export

-when criticality is achieved.

Reactors are listed as being built under the following circumstances:

1. Reactors regulated by the NRC

- when a construction permit is issued by NRC.

-when limited work authorization is issued by NRC.

2. Federal Government reactors

- when ground is broken.

-when components are ordered.

-when a construction contract is awarded.

3. Reactors for export

- when an application for an export license is received by NRC.

- when reliable information is received relating to the fabrication of reactor components. 
Reactors are listed as planned under the following circumstances:

1. Reactors regulated by NRC

-when a public announcement that includes the principal vendor supplier is made by the sponsoring organization.

- when an application for a construction permit is received by NRC.

2. Federal Government reactors

- when a public announcement is made by the agency involved.

- when the project is otherwise appropriately authorized.

3. Reactors for export

- when a public announcement that includes principal contractor and reactor type is made.

- when NRC receives information that a U.S. reactor manufacturer is proceeding with preconstruction design and development on the basis of a letter of intent.

Reactors are considered to be shutdown or dismantled under the following circumstances:

1. Reactors regulated by NRC

- when the licensee has applied to the Commission for authority to surrender a license voluntarily and to dismantle the facility and dispose of its component parts. A reactor shut down because of technical problems, modifications, or refueling continues to be listed as operable.

2. Federal Government reactors

- when the facility has ceased operation and the agency has declared officially that the agency does not intend to operate the reactor further. However, within this category, a few reactors are identified as being in standby mode, the condition in which documentary authorization exists to maintain the reactor for possible future operation.

3. Reactors for export

- when the plant is officially declared shut down by the owner and taken out of operation permanently.

Reactors and facilities shutdown or dismantled are listed in Section 3, Reactors and Facilities Shutdown or Dismantled.
The statistical summary (Table 2) shows the number of reactors in every category except critical facilities. Shutdown and dismantled reactors in these categories are included since such facilities have made significant contributions to reactor technology.

The reactor table column headings are defined or explained as follows:

Location-the city and state or country where located originally. For a portable facility or one that has been relocated, the most recent location is given.

Principal nuclear contractor, operator, designer, shipbuilder-the abbreviations are spelled out in Table 3, which appears just before the reactor tables.

Type-based on coolant, moderator, and neutron energy.

Power-MD Capacity [MW(e)] is the maximum dependable capacity (net electrical output to grid) for plants having an operating history. Otherwise, it is the design capacity. Licensed power and authorized power are given where appropriate.

Designation-the common name, abbreviation, or acronym used for the facility. For the naval reactors, it is the hull number.

Date columns-the initial criticality date, year of operation, and year of shutdown are given as appropriate.

Nuclear Reactors Built, Being Built, or Planned (DOE/OSTI-8200-R55) is sponsored by the DOE Office of Nuclear Energy, LaRue E. Moxley, Program Officer.

The participation and assistance of many individuals, agencies, and companies in providing data and updating the entries in this revision are gratefully acknowledged. Comments and suggestions about this publication are welcome.

To ensure that the wide range of information included in this publication will continue to be timely and accurate, please direct any information related to updating the items to William F. Simpson, Jr., Office of Scientific and Technical Information, Scientific and Technical Publications Branch, P.O. Box 62, Oak Ridge, TN 37831, telephone: (615) 576-1228. Questions of a technical nature should be addressed to Lamar Cason at the same address. 


\section{CONTENTS}

Preface

Map. Commercial Nuclear Power Reactors in the United States

viii

Table 1. Commercial Nuclear Power Reactors in Operation

in the United States

ix

Table 2. Statistical Summary of Nuclear Reactors $\quad$ xv

Table 3. Abbreviations of Contractors, Designers, Shipbuilders, and Facility Operators

xvi

\section{REACTORS AND FACILITIES}

\section{OPERABLE, BEING BUILT, OR PLANNED}

\section{Part I. Civilian Reactors (Domestic)}

1. Power Reactors

A. Central-Station Electric Power

B. Dual-Purpose Plants (No reactors currently in this category)

C. Propulsion (Maritime) (No reactors currently in this category)

2. Experimental Power-Reactor Systems
A. Electric-Power Systems
B. Space Nuclear Auxiliary Power (SNAP)
C. Space Propulsion (No reactors currently in this category)

3. Test, Research, and University Reactors
A. General Irradiation Test
B. High-Power Research and Test
C. Safety-Research and Test
D. General Research
E. University Research and Teaching

\section{Part II. Production Reactors}

1. Materials Production

2. Process Development (No reactors currently in this category) 


\section{CONTENTS (Continued)}

Part III. Military Reactors

1. Defense Power-Reactor Applications

A. Remote Installations (No reactors currently in this category)

B. Propulsion (Naval)

2. Developmental Power

A. Electric-Power Experiments and Prototypes (No reactors currently in this category)

B. Propulsion Experiments and Prototypes

3. Test and Research

A. Test (No reactors currently in this category)

B. Research

Part IV. Export Reactors

1. Power Reactors

A. Central-Station Electric Power Plants

B. Propulsion (No reactors currently in this category)

2. Test, Research, and Teaching

A. General Irradiation Test

B. General Research

C. University Research and Teaching

Part V. Critical Assemblies

1. Civilian

2. Military

\section{REACTORS AND FACILITIES \\ SHUTDOWN OR DISMANTLED}

\section{Part I. Civillan Reactors (Domestic)}

1. Power Reactors

A. Central-Station Electric Power

B. Dual-Purpose Plants

C. Propulsion (Maritime)

2. Experimental Power-Reactor Systems 25

A. Electric-Power Systems 25

B. Space Nuclear Auxiliary Power (SNAP) 27

C. Space Propulsion

3. Test, Research, and University Reactors 30
A. General Irradiation Test
B. High-Power Research and Test
C. Safety-Research and Test
D. General Research
E. University Research and Teaching 


\section{CONTENTS (Continued)}

Part II. Production Reactors

1. Materials Production

2. Process Development

Part III. Military Reactors

1. Defense Power-Reactor Applications $\quad 41$

A. Remote Installations $\quad 41$

B. Propulsion (Naval) $\quad 42$

2. Developmental Power $\quad 43$

A. Electric-Power Experiments and Prototypes $\quad 43$

B. Propulsion Experiments and Prototypes

3. Test and Research
A. Test
44
B. Research

Part IV. Export Reactors

1. Power Reactors 44

A. Central-Station Electric Power $\quad 44$

B. Propulsion 45

2. Test, Research, and Teaching $\quad 45$

A. General Irradiation Test (No reactors currently in this category)
B. General Research

C. University Research and Teaching 46

Part V. Critical Assemblies

1. Civilian $\quad 46$

$\begin{array}{lr}\text { 2. Military } & 47\end{array}$

REACTOR INDEX $\quad 49$ 


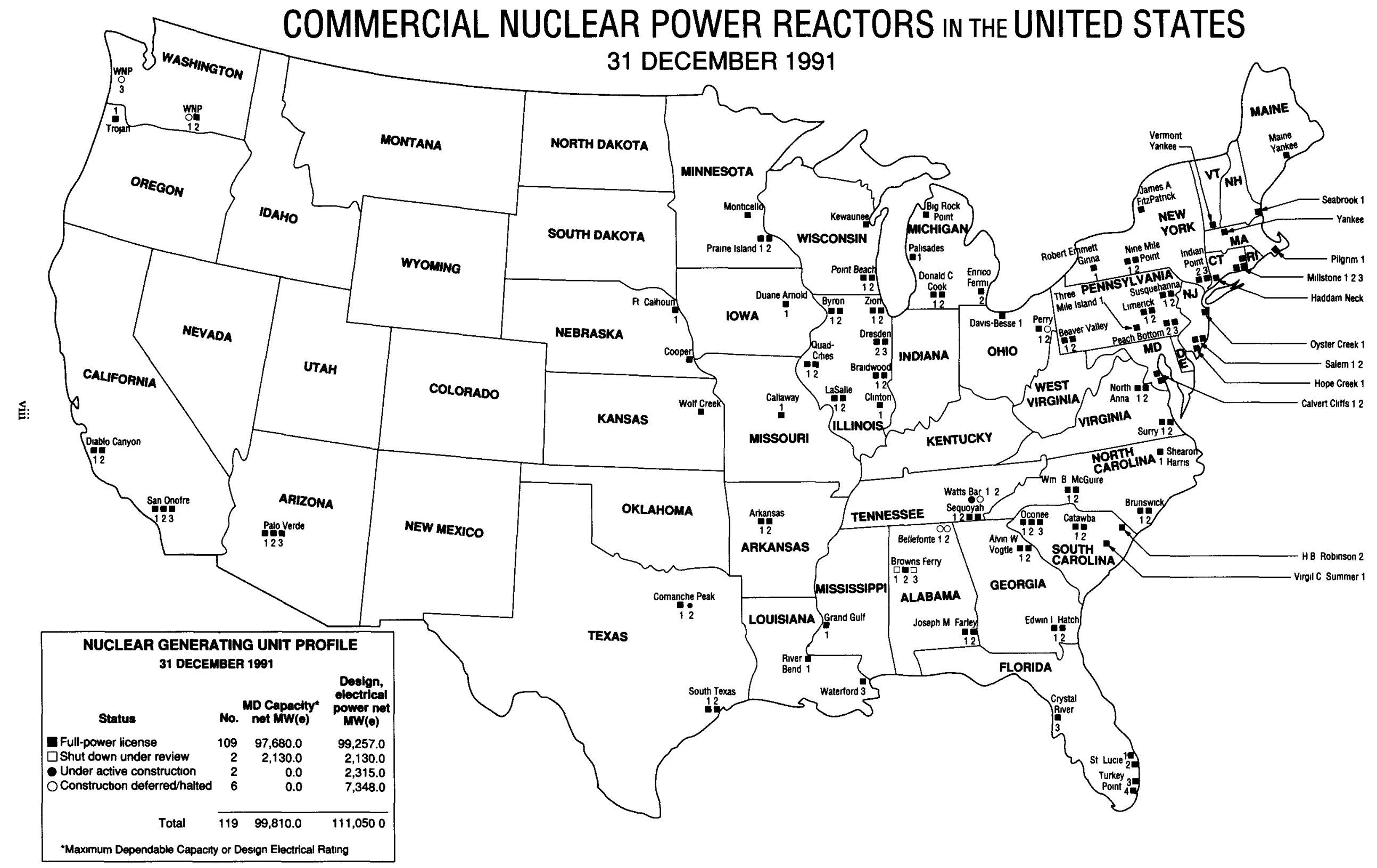

Bocause of spece IImitations, symbols do not reflect preciee locattons. DOE/OSTI-8200-R55 (Suppl.) DE92012402 
TABLE 1

COMMERCIAL NUCLEAR POWER REACTORS IN THE UNITED STATES AS OF 31 DEC 1991

ALABAMA

Decatur

Decatur

Decatur

Dothan

Dothan

Scousboro

Scottsboro

Subtotal

ARIZONA

Wintersburg

Wintersburg

Wintersburg

ARKANSAS

Russellville

Russellville

Subrotal

CALIFORNIA

Diablo Canyon

Diablo Canyon

San Clemente

San Clemente

Sen Clemente

Subtotal

CONNECTICUT

Haddam Neck

Browns Ferry Nuclear

Power Station, Unit 1

Browns Ferry Nuclear

Power Station, Unit 2

Browns Ferry Nuclear

Power Station, Unit 3

Joseph M. Farley

Nuclear Plant, Unit 1

Joseph M. Farley

Nuclear Plant, Unit 2

Bellefonte Nuclear

Plant, Unit 1

Bellefonte Nuclear

Plant, Unit 2

SDUR

1065.0

FPL

1065.0

SDUR

1065.0

FPL $\quad 825.0$

DESIGN,

ELECTRICAL

CAPACITY, POWER NET

MW(e)

LICENSEE

OR ESTI-

MATED

STARTUP

DATE

7308

7407

7608

7708

FPL $\quad \mathbf{8 2 4 . 0}$

Operating $\mathrm{CO}_{0}$.

Southern Nuclear

Operating Co.

$\begin{array}{ll}\mathrm{CDH} & 0.0\end{array}$

Tennessee Valley

Authority

Tennessee Valley

Authority

8105

Indef.

Indef.

$\begin{array}{ll}4844.0 & 7323.0\end{array}$

1221.0

1270.0

Arizona Public

Service Co.

8505

Generating Station,

Unit 1

Palo Verde Nuclear

Generating Station,

Unit 2

Palo Verde Nuclear

Generating Station,

Unit 3

FPL

1221.0

1270.0

Arizona Public

Service Co.

8604

FPL

1304.0

1270.0

Arizona Public

Service Co.

8710

3746.0

3810.0

Arkansas Nuclear One,

Unit 1

FPL

836.0

850.0

Entergy Operations

Inc.

FPL

858.0

912.0

Entergy Operations

Inc.

7408

7812

Unit 2

1694.0

1762.0

Diablo Canyon Nuclear Power Plant, Unit 1

Diablo Canyon Nuclear

Power Plant, Unit 2

San Onofre Nuclear

Generating Station,

Unit 1

San Onofre Nuclear

Generating Station

Unit 2

San Onofre Nuclear

Generating Station,

Unit 3

FP

1073.0

1086.0

Pacific Gas \&

Electric Co.

$\begin{array}{ll}\text { FPL } & 1079.0\end{array}$

1119.0

Pacific Gas \&

$\begin{array}{ll}\text { FPL } & 436.0\end{array}$

436.0

Electric Co.

Southem Califomia

Edison Co.

FPL

1070.0

1070.0

Southem Califomia

Edison $\mathrm{Co}$.

FPL

1080.0

1080.0

Southem Califomia

Edison Co.

8404

8508

6706

8207

8308

4738.0

4791.0

Haddam Neck Plant

FPL

569.0

582.0

Connecticul Yankee

6707 
TABLE 1 (Continued)

SITE

Waterford

Waterford

Waterford

Subtotal

\section{FLORIDA}

Flonda City

Flonda City

Font Pierce

Fort Pierce

Red Level

Subtotal

\section{GEORGIA}

Baxley

Baxley

Waynesboro

Waynesboro

Subtotal

\section{ILLINOIS}

Braldwood

Brasdwood

Byron

Byron

Clinton

Cordova

Cordova

Morns

Morns

Seneca

Seneca

Zion

Zion

Subtotal
PLANT NaMe

Millstone Nuclear Power

Station, Unit 1

Millstone Nuclear Power

Station, Unit 2

Millstone Nuclear Power

Station, Unit 3

$$
\begin{aligned}
& \text { Turkey Point Plant, } \\
& \text { Unit } 3 \\
& \text { Turkey Point Plant, } \\
& \text { Unit } 4 \\
& \text { St. Lucie Plant, } \\
& \text { Unit } 1 \\
& \text { St. Lucie Plant, } \\
& \text { Unit } 2 \\
& \text { Crystal River Nuclear } \\
& \text { Plant, Unt } 3
\end{aligned}
$$
Edwin I Hatch Nuclear Plant, Unt 1
Edwon I Hatch Nuclesr
Plant, Unit 2
Alvin W Vogtle
Nuclear Plant, Unt 1
Alvin W Vogtle
Nuclear Plant, Unt 2

Braidwood Station, Unit 1

Braidwood Station,

Unit 2

Byron Station, Unit 1

Byron Station, Unt 2

Clinton Power Station,

Unit 1

Quad Citues Station,

Unit 1

Quad-Cittes Station,

Unit 2

Dresden Nuclear Power

Station, Unit 2

Dresden Nuclear Power

Station, Unit 3

La Salle County Station,

Unit 1

La Salle County Station, Unit 2

Zion Nuclear Plant,

Unit 1

Zion Nuclear Plant,

Unt 2
STATUS

FPL

CAPACITY,

DESIGN, NET MW(e)

ELECTRICAL

POWER NET

MW(e)

LICENSEE

OR ESTI-

MATED

STARTUP

DATE

7010

FPL $\quad 8570$

6600

Northeast Nuclear

Energy Co

8700 Northeast Nuclear

Energy Co

FPL

11420

11540

Northeast Nuclear

Energy Co

32220

32660

FPL

6660

6930

Flonda Power \&

Light Co

FPL $\quad 666$

6930

Flonda Power \&

Light Co

FPL $\quad 839$

8300

Flonda Power \&

Lught Co

FPL $\quad 839$

8300

Flonda Power \&

Light Co

FPL

8210

8250

Flonda Power Corp

38710

FPL

38310

7760 Georgia Power Co

FPL

7610

7840

Georgia Power Co

FPL

11010

11010

Georgia Power Co

FPL

11010

11010

Georgia Power Co

37130

37620

FPL

11200

11200

Commonwealth

Edison Co

FPL $\quad 11200$

11200

Commonwealth

Edison Co

FPL $\quad 11290$

11200

Commonwealth

Edison $\mathrm{Co}$

FPL $\quad 11290$

11200

Commonwealth

Edison Co

FPL $\quad 9500$

9330

Illunoss Power Co

7890

Commonwealth

Edison Co

FPL $\quad 7690$

7890

Commonwealth

Edison Co

FPL $\quad 7720$

7940

Commonwealth

Edison Co

FPL $\quad 7730$

7940

Commonwealth

Edison Co

FPL $\quad 10360$

10780

Commonwealth

Edison Co

FPL $\quad 10360$

10780

Commonwealth

Edison Co

FPL

10400

10400

Commonwealth

Edison Co

FPL

10400

10400

Commonwealth

Edison Co

128150
7409

7807

8703

8903

7510

8601

7210

7306

7604

8306

7701

8705

8803

8502

8701

8702

7110

7204

7001

7101

8206

8403

7306

7312 
TABLE 1 (Continued)

STIE

PLANT NAME

STATUS

$\begin{array}{cc}\text { MD } & \text { ELECTRICAL } \\ \text { CAPACITY, } & \text { POWER NET }\end{array}$

LICENSEE

STARTUP

OR ESTI-

MATED

NET MW(e) MW(e)

STARTUP

DATE

IOWA

Palo

Duane Amold Energy

Center, Unit 1

Subtotal

KANSAS

Burlington

Subrotal

LOUISIANA

St. Francisville

Taft

Subtotal

MAINE

Wiscasset

Subtotal

\section{MARYLAND}

Lusby

Lusby

Subtotal

MASSACHUSETTS

Plymouth

Rowe

Subtotal

MICHIGAN

Big Rock Point

Bridgman

Bridgman

Newport

South Haven

Subtotal

\section{MINNESOTA}

Monticello

Red Wing

Red Wing

Subtotal

Power Plant

Calvert Cliffs Nuclear

Power Plant, Unit 1

Calvert Cliffs Nuclear

Power Plant, Unit 2

Station, Unit 1 Station Plant

Power Plant, Unit 1

Power Plant, Unit 2

Enrico Fermi Atomic

Power Plant, Unit 2

Palisades Nuclear

Plant, Unit 1

Monticello Nuclear

Generating Plant

Prairie Island Nuclear

Generating Plant,

Unit 1

Prairie Island Nuclear

Generating Plant.

Unit 2
FPL

\begin{tabular}{|c|c|c|c|}
\hline 515.0 & 538.0 & $\begin{array}{l}\text { Iowa Electric Light \& } \\
\text { Power Co. }\end{array}$ & 7403 \\
\hline 515.0 & 538.0 & & \\
\hline 1128.0 & 1170.0 & $\begin{array}{r}\text { Kansas Gas \& } \\
\text { Electric Co. }\end{array}$ & 8505 \\
\hline 1128.0 & 1170.0 & & \\
\hline 936.0 & 936.0 & Gulf States Utilities Co. & 8510 \\
\hline 1075.0 & 1104.0 & $\begin{array}{l}\text { Entergy Operations } \\
\text { Inc. }\end{array}$ & 8503 \\
\hline 2011.0 & 2040.0 & & \\
\hline
\end{tabular}

FPL

810.0

825.0

Maine Yankee Atomic

7210

810.0

825.0

FPL
FPL

825.0

845.0

Baltimore Gas \&

825.0

845.0

ectric Co.

1650.0

1690.0

FPL

670.0

655.0

Boston Edison Co.

7206

Yankee Nuclear Power

FPL

167.0

175.0

Yankee Atomic

Electric Co.

6008

837.0

830.0

Big Rock Point Nuclear

Donald C. Cook Nuclear

Donald C. Cook Nuclear

FP

69.0

72.0

Consumers Power Co.

6209

FPL $\quad 1020$.

1030.0

Indiana and Michigan

7501

FPL $\quad 1060.0$

1100.0

Electric Co.

FPL $\quad 1093.0$

1093.0

Indiana and Michigan

7803

Electric Co.

FPL 730 .

805.0

Consumers Power Co.

8506

7105

3972.0

4100.0

$\begin{array}{ll}\text { FPL } & 536.0 \\ \text { FPL } & 503.0 \\ \text { FPL } & 500.0\end{array}$

1539.0
545.0 Northem States
Power Co.

$530.0 \quad$ Northem States

Power Co.

$530.0 \quad$ Northem States

Power Co.
7012

7312

7412 
TABLE 1 (Continued)

PLANT NAME

Grand Gulf Nuclear
Station, Unit 1

Subtotal

\section{MISSOURI}

Fulton

Subtotal

NEBRASKA

Brownville

Fort Calhoun

Subtotal

NEW HAMPSHIRE

Seabrook

Subtotal

NEW JERSEY

Salem

Salem

Salem

Toms River

Subtotal

NEW YORK

Buchanan

Buchanan

Ontario

Scribe

Scriba

Scrib

Subtotal

NORTH CAROLINA

Bonsal

Cowans Ford

Dam

Cowans Ford

Dam

Southpon
Callaway Plant, Unit 1

Cooper Nuclear Station

Fort Calhoun Station, Unit 1

\section{Seabrook Nuclear \\ Station, Unit 1}

$$
\begin{aligned}
& \text { Hope Creek Nuclear } \\
& \text { Generating Station, } \\
& \text { Unit } 1 \\
& \text { Salem Nuclear } \\
& \text { Generating Station, } \\
& \text { Unit } 1 \\
& \text { Salem Nuclear } \\
& \text { Generating Station, } \\
& \text { Unit } 2 \\
& \text { Oyster Creek Nuclear } \\
& \text { Power Plant, Unit } 1
\end{aligned}
$$

Indian Point Station, Unit 2

Indian Point Station,

Unit 3

Robert Emmen Ginna

Nuclear Power Plant,

Unit 1

James A. FitzPatrick

Nuclear Power Plant

Nine Mile Point Nuclear

Station, Unit 1

Nine Mile Point Nuclear

Station, Unit 2

Shearon Harris Nuclear Power Plant, Unit 1

William B. McGuire

Nuclear Station,

Unit 1

William B. McGuire

Nuclear Station,

Unit 2

Brunswick Steam

Electric Plant, Unit 1

\section{MD}

CAPACITY,

STATUS
FPL

1142.0

1250.0

DESIGN,

ELECTRICAL

POWER NET

LICENSEE

STARTUP

OR ESTI-

MATED

STARTUP

DATE

8208 Inc.

$\begin{array}{lll}1142.0 & 1250.0 & \text { Inc. }\end{array}$

FPL

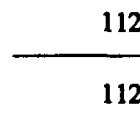

FPL

764.0

778.0

Nebraska Public

7402

FPL

478.0

478.0

Power Distric

Omaha Public

Power District

7308

1242.0

1256.0

FPL

1198.0

1198.0

Public Service Co of New Hampshire

8906

1198.0

1198.0

FPL

1067.0

1067.0

Public Service

Electric \& Gas Co.

FPL.

1106.0

1115.0

Public Service

Electric \& Gas Co.

FPL

1106.0

1115.0

Public Service

Electric \& Gas Co.

FPL

620.0

650.0

GPU Nuclear Corp.

6905

3899.0

3947.0

FPL

849.0

873.0

Consolidated Edison

7305

FPL $\quad 1000.0$

965.0

Co. of New York, Inc.

FPL $\quad 470.0$

New York

$470.0 \quad$ Rochester Gas

Electric Corp.

FPL $\quad 794.0$

816.0 New York Power Authority

FPL $\quad 610.0$

620.0

Niagara Mohawk

Power Corp.

FPL

1080.0

1080.0

Niagara Mohawk

Power Corp.

4803.0

4824.0

FPL

915.0

900.0

Carolina Power \&

8701

FPL

1150.0

1180.0

Light Co.

Duke Power Co.

8108

FPL

1150.0

1180.0

Duke Power Co.

8305

FPL

790.0

821.0

Carolina Power \&

Light Co. 
TABLE 1 (Continued)

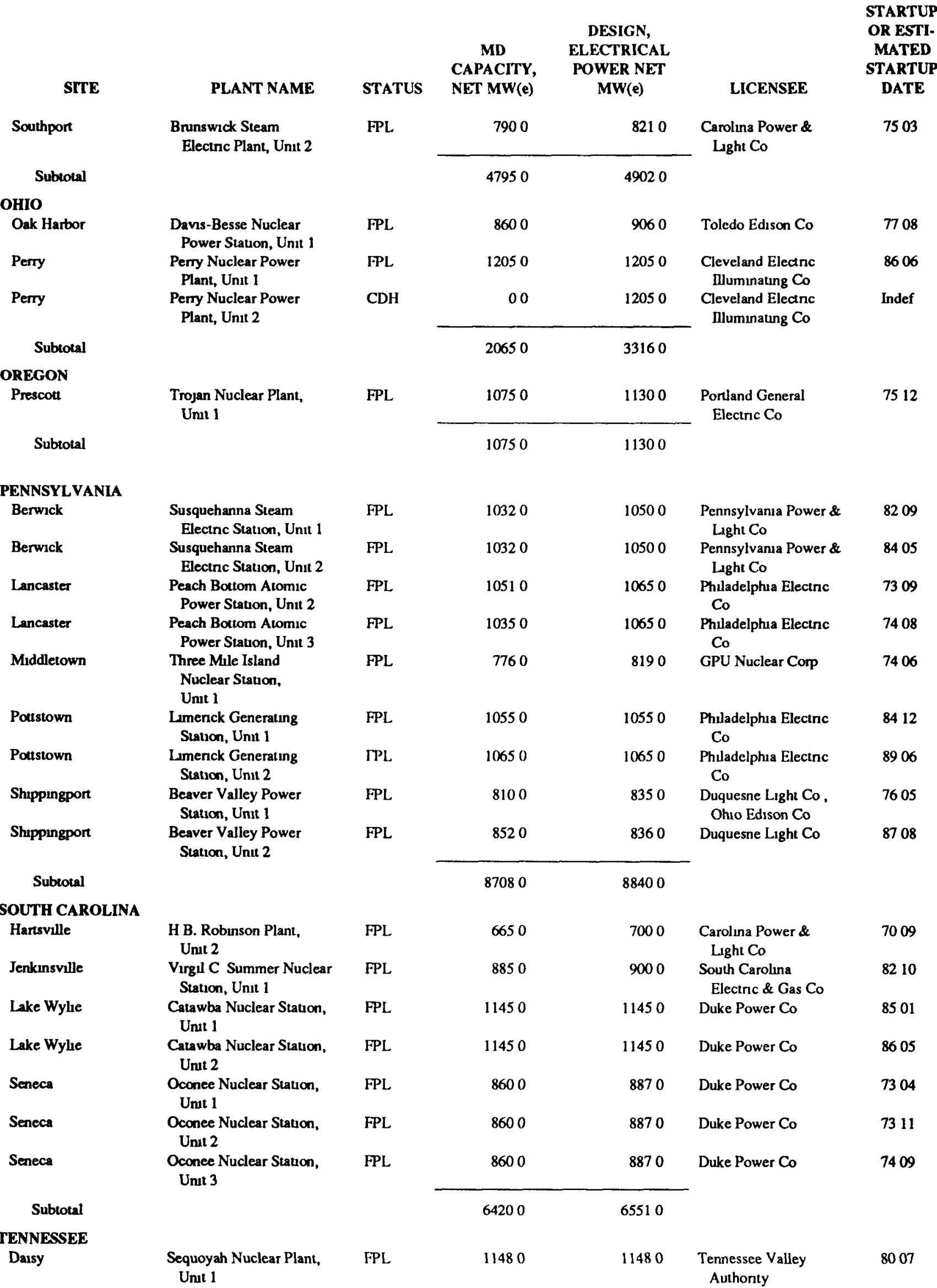


TABLE 1 (Continued)

Dasyy

Spnng City

Spang City

Subtotal

TEXAS

Bay Caty

Bay City

Glen Rose

Glen Rose

Subtotal

VERMONT

Vemon

Subtotal

\section{VIRGINIA}

Gravel Neck

Gravel Neck

Muneral

Mineral

Subtotal

\section{WASHINGTON}

Ruchland

Ruchland

Satsop

Subtotal

WISCONSIN

Carkon

Two Creeks

Two Creeks

Subtotal

Total

\section{PLANT NAME}

Sequoyah Nuclear Plant,

Umt 2

Watts Bar Nuclear Plant. Unit 1

Watts Bar Nuclear Plant, Unt 2

South Texas Project.

Unit 1

South Texas Project,

Unt 2

Comanche Peak Steam

Electnc Station, Unit I

Comanche Peak Steam

Electnc Station, Unit 2

Vermont Yankee Nuclear Power Station

Surry Power Station, Unit 1

Surry Power Station, Unit 2

North Anna Power

Station, Untt 1

North Anna Power

Station, Unit 2

Washington Nuclear Project, Unit 1

Washington Nuclcar Project, Unit 2

Washington Nuclear Project, Unit 3

$$
\begin{aligned}
& \text { Kewaunee Nuclear } \\
& \text { Power Plant } \\
& \text { Point Beach Nuclear } \\
& \text { Plant, Unit 1 } \\
& \text { Point Beach Nuclear } \\
& \text { Plant, Unt } 2
\end{aligned}
$$

\section{STATUS}

MD

CAPACITY, NET MW(e)

FPL

11480

UC

$\mathrm{CDH}$

PI

FPL

FPL

11480
00
00
DESIGN, ELECTRICAL POWER NET MW(e)

00

11480
11650
11650
46260

LICENSEE

22960

12500

12500

12500

12500

11500

11500

UC

00

11500

36500

FL

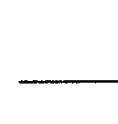

5040

7810

7810

9150

FPL

FPL

$$
9150
$$

\begin{tabular}{|c|c|c|c|}
\hline I PL & 5030 & 5350 & $\begin{array}{l}\text { Wisconsin Public } \\
\text { Service Corp }\end{array}$ \\
\hline IPI & 4850 & 4970 & $\begin{array}{l}\text { Wisconsin Electric } \\
\text { Power Co }\end{array}$ \\
\hline \multirow[t]{2}{*}{ IPL } & 4850 & 4970 & $\begin{array}{l}\text { Wisconsin Electnc } \\
\text { Power Co }\end{array}$ \\
\hline & 14730 & 15290 & \\
\hline
\end{tabular}

33920

())

CIII
IPI
CDII

00
10950
00

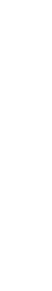

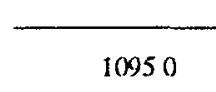

CDH, Construction Deferred/l lalted

SDUR, Shut Down Under Revicw
Tennessee Valley

Authonty

Tennessee Valley

Authonty

Tennessee Valley

Authonty

STARTUP

OR ESTI.

MATED

STARTUP

DATE

8111

Indef

Indef

8803

8902

9004

9212

7203

Nuclear Power Corp

7207

7303

7804

8006

Indef Power Supply System

Washington Public Power Supply System

Washington Public Power Supply System

8401

Indef

7403

7011

7205
FPL, Full-Power License UC, Under Actıve Constructuon 
TABLE 2

\section{STATISTICAL SUMMARY OF NUCLEAR REACTORS AS OF DECEMBER 1991}

$\begin{array}{ccc}\text { Being } & & \begin{array}{c}\text { Shut down } \\ \text { or } \\ \text { Operable }\end{array} \\ \text { built } & \text { Planned dismantled }\end{array}$

I. CIVILIAN REACTORS (DOMESTIC)

1. Power Reactors

A. Central-Station Electric Power Plants

111

8

1-Purpose Plants

C. Propulsion (Maritime)

2. Experimental Power-Reactor Systems

A. Electric-Power Systems

B. Space Nuclear Auxiliary Power

C. Space Propulsion (Rover)

3. Test, Research, and University Reactors

A. General Irradiation Test

B. High-Power Research and Test

C. Safety-Research and Test

D. General Research

E. University Research and Teaching

1

PRODUCTION REACTORS

1. Materials Production

2. Process Development

III. MILITARY REACTORS

1. Defense Power-Reactor Applications

A. Remote Installations

B. Propulsion (Naval)

145

22

6

2. Developmental Power

A. Electric-Power Experiments and Prototypes

B. Propulsion Experiments and Prototypes

3. Test and Research

A. Test

B. Research

1. Power Reactors

A. Central-Station Electric Power Plants

B. Propulsion

2. Test, Research, and Teaching

A. General Irradiation Test

B. General Research

C. University Research and Teaching

\section{REACTORS FOR EXPORT}

ismantled 


\section{TABLE 3 ABBREVIATIONS OF CONTRACTORS, DESIGNERS, SHIPBUILDERS, AND FACILITY OPERATORS}

The definitions of the following abbreviations that have been used in this volume contan references to current and historical corporate and government structure.

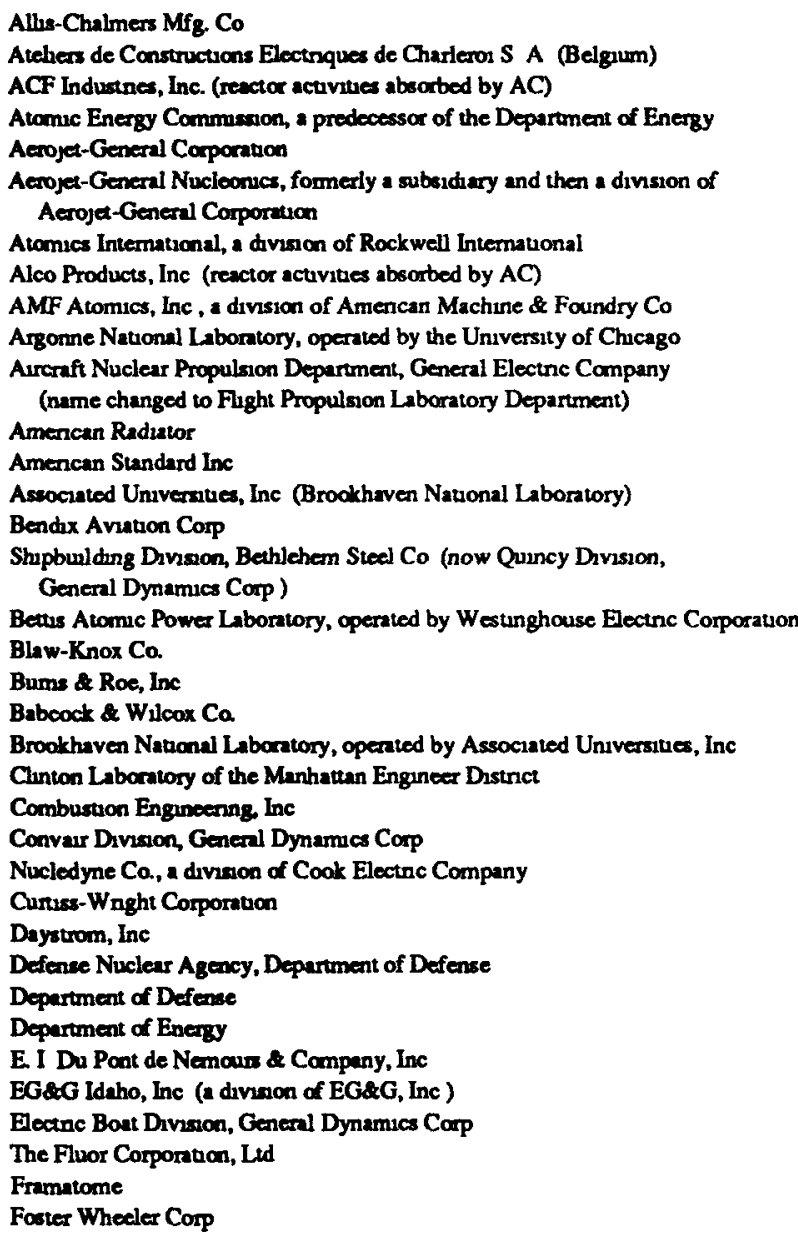

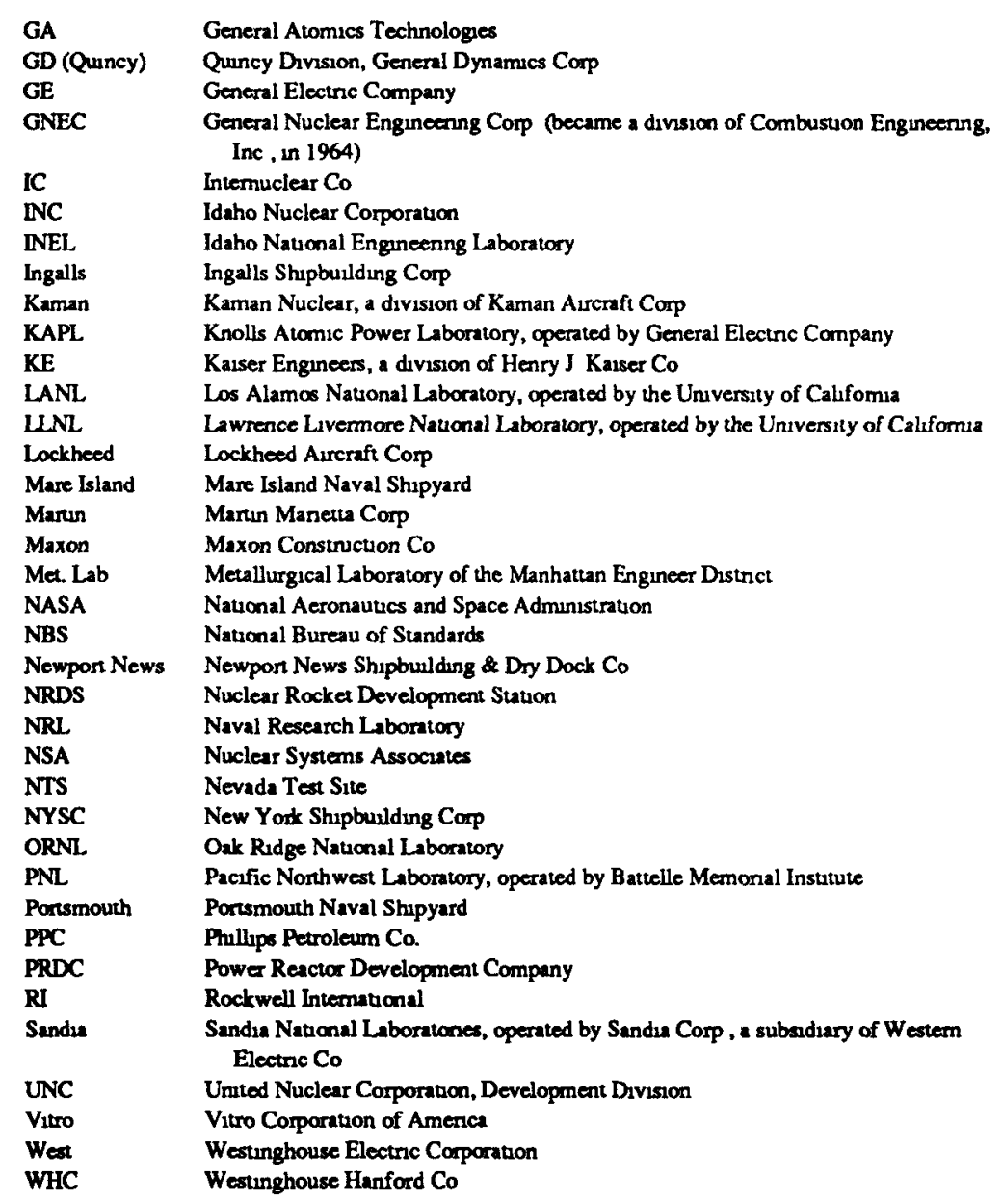




\section{REACTORS AND FACILITIES OPERABLE, BEING BUILT, OR PLANNED}




\section{REACTORS AND FACILITIES OPERABLE, BEING BUILT, OR PLANNED}

\section{POWER REACTORS}

\section{A. Central-Station Electric Power Plants}

Name (licensee) [docket number]

\section{OPERABLE}

Alvin W. Vogtle Nuclear Plant, Unit 1 (Georgia Power Co.) [50-424]

Alvin W. Vogtle Nuclear Plant, Unit 2

(Georgia Power Co.) [50-425]

Arkansas Nuclear One, Unit 1 (Entergy

Operations Inc.) [50-313]

Arkansas Nuclear One, Unit 2 (Entergy

Operations Inc.) [50-368]

Beaver Valley Power Station, Unit 1 (Duqueste Light Co., Ohio Edison Co.) [50-334]

- Beaver Valley Power Station, Unit 2

(Duquesne Light Co.) [50-412]

Big Rock Point Nuclear Plant

(Consumers Power Co.) [50-155]

Braidwood Station, Unit 1

(Commonwealth Edison Co.) [50-456]

Braidwood Station, Unit 2

(Commonwealth Edison Co.) [50-457]

Browns Ferry Nuclear Power Station, Unit

(Tennessee Valley Authority) [50-259]

Browns Ferry Nuclear Power Station, Unit 2 (Tennessee Valley Authority) [50-260]

Browns Ferry Nuclear Power Station, Unit 3 (Tennessee Valley Authority) [50-296]

Bunswick Steam Electric Plant, Unit 1

(Carolina Power \& Light Co.) [50-325]

Brunswick Steam Electric Plant, Unit 2

(Carolina Power \& Light Co.) [50-324]

Byron Station, Unit 1

(Commonwealth Edison Co.) [50-454]

Byron Station, Unit 2

(Commonwealth Edison Co.) [50-455]

Callaway Plant, Unit 1 (Union Electric Co.) [50-483]

Calvert Cliffs Nuclear Power Plant, Unit 1

(Baltimore Gas \& Electric Co.) [50-317]

\begin{tabular}{|c|c|c|c|c|c|}
\hline \multirow[b]{2}{*}{ Location } & \multirow[b]{2}{*}{$\begin{array}{l}\text { Principal } \\
\text { nuclear } \\
\text { contractor }\end{array}$} & \multirow[b]{2}{*}{ Type } & \multicolumn{2}{|c|}{ Powwer } & \multirow[b]{2}{*}{$\begin{array}{c}\text { Initial } \\
\text { criticality } \\
\text { (yr mo) }\end{array}$} \\
\hline & & & $\begin{array}{l}\text { MD capacity } \\
\text { net } M W(e)\end{array}$ & $\begin{array}{l}\text { Licensed } \\
\text { MW(t) }\end{array}$ & \\
\hline Waynesboro, GA & West. & $\begin{array}{l}\text { Pressurized } \\
\text { water }\end{array}$ & 1101.0 & 3411.0 & 8703 \\
\hline Waynesboro, GA & West. & $\begin{array}{l}\text { Pressurized } \\
\text { water }\end{array}$ & 1101.0 & 3411.0 & 8903 \\
\hline Russellville, AR & B\&W & $\begin{array}{l}\text { Pressurized } \\
\text { water }\end{array}$ & 836.0 & 2568.0 & 7408 \\
\hline Russellville, AR & Comb. & $\begin{array}{l}\text { Pressurized } \\
\text { water }\end{array}$ & 858.0 & 2815.0 & 7812 \\
\hline Shippingpor, PA & West. & $\begin{array}{l}\text { Pressurized } \\
\text { water }\end{array}$ & 810.0 & 2660.0 & 7605 \\
\hline Shippingport, PA & West. & $\begin{array}{l}\text { Pressurized } \\
\text { water }\end{array}$ & 852.0 & 2660.0 & 8708 \\
\hline Big Rock Point, MI & GE & Boiling water & 69.0 & 240.0 & 6209 \\
\hline Braidwood, IL & West. & $\begin{array}{l}\text { Pressurized } \\
\text { water }\end{array}$ & 1120.0 & 3411.0 & 8705 \\
\hline Braidwood, IL & West. & $\begin{array}{l}\text { Pressurized } \\
\text { water }\end{array}$ & 1120.0 & 3411.0 & 8803 \\
\hline Decatur, AL & GE & Boiling water & 1065.0 & 3293.0 & 7308 \\
\hline Decatur, AL & GE & Boiling water & 1065.0 & 3293.0 & 7407 \\
\hline Decatur, AL & GE & Boiling water & 1065.0 & 3293.0 & 7608 \\
\hline Southport, NC & GE & Boiling water & 790.0 & 2436.0 & 7610 \\
\hline Southpor, NC & GE & Boiling water & 790.0 & 2436.0 & 7503 \\
\hline Byron, IL & West. & $\begin{array}{l}\text { Pressurized } \\
\text { water }\end{array}$ & 1129.0 & 3411.0 & 8502 \\
\hline Byron, IL & West. & $\begin{array}{l}\text { Pressurized } \\
\text { water }\end{array}$ & 1129.0 & 3411.0 & 8701 \\
\hline Fulton, MO & West. & $\begin{array}{c}\text { Pressurized } \\
\text { water }\end{array}$ & 1120.0 & 3565.0 & 8410 \\
\hline Lusby, MD & Comb. & $\begin{array}{l}\text { Pressurized } \\
\text { water }\end{array}$ & 825.0 & 2700.0 & 7410 \\
\hline
\end{tabular}

PART I CIVILIAN REACTORS (DOMESTIC)

itial

mo)

Comment
Shut down 6/1/85: administrative hold to resolve various TVA and NRC concems.

Restarted 5/23/91.

Shut down 3/3/85: administrative hold to resolve various TVA and NRC concerns. 


\section{A. Central-Station Electric Power Plants (Continued)}

Name (licensee) [docket number]

OPERABLE (Continued)

Calven Cliffs Nuclear Power Plant, Unit 2

(Baltimore Gas \& Electric Co.) [50-318]

Catawba Nuclear Station, Unit 1

(Duke Power Co.) [50-413]

Catawba Nuclear Station, Unit 2

(Duke Power Co.) [50-414]

Clinton Power Station, Unit 1

(llinois Power Co.) [50-461]

Comanche Peak Steam Electric Station, Unit 1

(Texas Utilities Generating $\mathrm{Co}_{0}$ ) [50-445]

Cooper Nuclear Station (Nebrask

Public Power District) [50-298]

Crystal River Nuclear Plant, Unit 3

(Florida Power Corp.) [50-302]

$N$ Davis-Besse Nuclear Power Station,

Unit 1 (Toledo Edison Co.) [50-346]

Diablo Canyon Nuclear Power Plant, Unit 1

(Pacific Gas \& Electric Co.) [50-275]

Diablo Canyon Nuclear Power Plant, Unit 2

(Pacific Gas \& Electric Co.) [50-323]

Donald C. Cook Nuclear Power Plant, Unit 1

(Indiana and Michigan Electric Co.) [50-315]

Donald C. Cook Nuclear Power Plant, Unit 2

(Indiana and Michigan Elecaric Co.) [50-316]

Dresden Nuclear Power Station, Unit 2

(Commonwealth Edison Co.) [50-237]

Dresden Nuclear Power Station, Unit 3

(Commonwealth Edison Co.) [50-249]

Duane Arnold Energy Center, Unit 1 (Iows

Electric Light \& Power Co.) [50-331]

Edwin I. Hatch Nuclear Plant, Unit

(Georgia Power Co.) [50-321]

Edwin I. Hatch Nuclear Plant, Unit 2

(Georgia Power Co.) [50-366]

Enrico Fermi Atomic Power Plant,

Unit 2 (Detroit Edison Co.) [50-341]

Fon Calhoun Station, Unit 1 (Omaha

Public Power District) [50-285]

Grand Gulf Nuclear Station, Unit 1

(Entergy Operations Inc.) [50-416] nudear

contractor

Lusby, MD

Lake Wylie, SC

Comb.

Pressurized

water

Lake Wylie, SC

Clinton, IL

Glen Rose, TX

Brownville, NE

Red Level, FL

Oak Harbor, $\mathrm{OH}$

Diablo Canyon, CA

Diablo Canyon, CA

Bridgman, MI

Bridgman, MI

Morris, IL

Morris, Il

Palo, IA

Baxley, GA

Baxley, GA

Newpon, MI

Fort Calhoun, NE

Pon Gibson, MS
West.

Pressurized

water

West. Pressurized

water

GE Boiling water

Pressurized

GE Boiling water

764.0

B\&W Pressurized

water

B\&W Pressurized

water

West. Pressurized

water

West. Pressurized

water

West. Pressurized

water

West. Pressurized

water

Boiling water

Boiling water

Boiling water

Boiling water

Boiling water

Boiling water

Comb.

Pressurized

water

GE

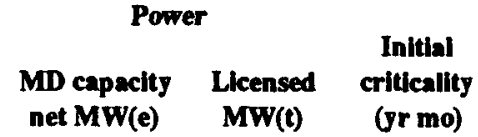

(or mo)

Comment

$\begin{array}{rlll}825.0 & 2700.0 & 7611 & \\ 1145.0 & 3411.0 & 8501 & \\ 1145.0 & 3411.0 & 8605 & \\ 950.0 & 2894.0 & 8702 & \\ 1150.0 & 3411.0 & 9004 & \begin{array}{l}\text { Full-power license, 4/17/90. } \\ \text { Commercial operation, 8/13/90. }\end{array} \\ 764.0 & 2381.0 & 7402 & \end{array}$

$821.0 \quad 2544.0$

$860.0 \quad 2772.0 \quad 7708$

$1073.0 \quad 3338.0 \quad 8404$

$\begin{array}{lll}1079.0 & 3411.0 & 8508\end{array}$

$\begin{array}{lll}1020.0 & 3250.0 & 7501\end{array}$

$1060.0 \quad 3411.0 \quad 7803$

$\begin{array}{lll}772.0 & 2527.0 & 7001\end{array}$

$\begin{array}{lll}773.0 & 2527.0 & 7101\end{array}$

$\begin{array}{lll}515.0 & 1658.0 & 7403\end{array}$

$\begin{array}{lll}750.0 & 2436.0 & 7409\end{array}$

$\begin{array}{lll}761.0 & 2436.0 & 7807\end{array}$

$\begin{array}{lll}1093.0 & 32920 & 8506\end{array}$

$\begin{array}{lll}478.0 & 1500.0 & 7308\end{array}$

$\begin{array}{lll}1142.0 & 3833.0 & 8208\end{array}$
Commercial operation, 8/13/90. 
H.B. Robinson Plant, Unit 2 (Carolina Power \& Light Co.) [50-261]

Haddam Neck Plant (Connecticut

Yankee Alomic Power Co.) [50-213]

Hope Creek Nuclear Generating Station, Unir 1

(Public Service Electric \& Gas Co.) [50-213]

Indian Point Station, Unit 2 (Consolidated

Edison Co. of New York, Inc.) [50-247]

Indian Point Station, Unit 3 (New

York Power Authority) [50-286]

James A. FitzPatrick Nuclear Power

Plant (New York Power Authority) [50-333]

Joseph M. Farley Nuclear Planh, Unit 1

(Southem Nuclear Operating Co.) [50-348]

Joseph M. Farley Nuclear Plant, Unit 2

(Southern Nuclear Operating Co.) [50-364]

Kewaunee Nuclear Power Plant

(Wisconsin Public Service Corp.) [50-305]

La Salle County Station, Unit 1

(Commonwealth Edison Co.) [50-373]

La Salle County Station, Unit 2

(Commonwealth Edison Co.) [50-374]

Limerick Generating Station, Unit 1

(Philadelphia Electric Co.) [50-352]

$\omega$ Limerick Generating Station, Unit 2

(Philadelphia Electric Co.) [50-353]

Maine Yankee Atomic Power Plant

(Maine Yankee Atomic Power Co.) [50-309]

Millstone Nuclear Power Station, Unit 1

(Northeast Nuclear Energy Co.) [50-245]

Millstone Nuclear Power Station, Unit 2

(Northeast Nuclear Energy Co.) [50-336]

Millstone Nuclear Power Station, Unit 3

(Northeast Nuclear Energy Co.) [50-423]

Monticello Nuclear Generating Plant

(Northem States Power Co.) [50-263]

Nine Mile Point Nuclear Station, Unit 1

(Niagara Mohawk Power Corp.) [50-220]

Nine Mile Point Nuclear Station, Unit 2

(Niagara Mohawk Power Corp.) [50-410]

North Anna Power Station, Unit 1

(Virginia Electric \& Power Co.) [50-338]

North Anna Power Station, Unit 2

(Virginia Electric \& Power Co.) [50-339]

Oconee Nuclear Station, Unit 1 (Duke Power Co.) [50-269]

Oconee Nuclear Station, Unit 2 (Duke Power Co.) [50-270]

Oconee Nuclear Station, Unit 3 (Duke Power Co.) [50-287]

\begin{tabular}{|c|c|c|c|c|c|}
\hline Hartsville, SC & West. & $\begin{array}{l}\text { Pressurized } \\
\text { water }\end{array}$ & 665.0 & 2300.0 & 7009 \\
\hline Haddam Neck, CT & Weat. & $\begin{array}{l}\text { Pressurized } \\
\text { water }\end{array}$ & 569.0 & 1825.0 & 6707 \\
\hline Salem, NJ & GE & Boiling water & 1067.0 & 3293.0 & 8606 \\
\hline Buchanan, NY & West. & $\begin{array}{l}\text { Pressurized } \\
\text { water }\end{array}$ & 849.0 & 2758.0 & 7305 \\
\hline Buchenan, NY & West. & $\begin{array}{l}\text { Pressurized } \\
\text { water }\end{array}$ & 1000.0 & 3025.0 & 7604 \\
\hline Scriba, NY & GE & Boiling water & 794.0 & 2436.0 & 7411 \\
\hline Dochan, AL & West. & $\begin{array}{l}\text { Pressurized } \\
\text { water }\end{array}$ & 825.0 & 2652.0 & 7708 \\
\hline Dothan, AL & West. & $\begin{array}{l}\text { Pressurized } \\
\text { water }\end{array}$ & 824.0 & 2652.0 & 8105 \\
\hline Cartion, WI & West. & $\begin{array}{l}\text { Pressurized } \\
\text { water }\end{array}$ & 503.0 & 1650.0 & 7403 \\
\hline Seneca, IL & GE & Boiling water & 1036.0 & 3323.0 & 8206 \\
\hline Seneca, IL & GE & Boiling water & 1036.0 & 3323.0 & 8203 \\
\hline Pottstown, PA & GE & Boiling water & 1055.0 & 3293.0 & 8412 \\
\hline Pottstown, PA & GE & Boiling water & 1065.0 & 3293.0 & 8912 \\
\hline Wiscasset, ME & Comb. & $\begin{array}{l}\text { Pressurized } \\
\text { water }\end{array}$ & 810.0 & 2630.0 & 7210 \\
\hline Waterford, CT & GE & Boiling water & 654.0 & 2011.0 & 7010 \\
\hline Waterford, CT & Comb. & $\begin{array}{l}\text { Pressurized } \\
\text { water }\end{array}$ & 857.0 & 2700.0 & 7510 \\
\hline Waterford, CT & West. & $\begin{array}{l}\text { Pressurized } \\
\text { water }\end{array}$ & 1142.0 & 3411.0 & 8601 \\
\hline Monticello, MN & GE & Boiling water & 536.0 & 1670.0 & 7012 \\
\hline Scriba, NY & GE & Boiling water & 610.0 & 1850.0 & 6909 \\
\hline Scriba, NY & GE & Boiling water & 1080.0 & 3323.0 & 8705 \\
\hline Mineral, VA & West. & $\begin{array}{l}\text { Pressurized } \\
\text { water }\end{array}$ & 915.0 & 2893.0 & 7804 \\
\hline Mineral, VA & West. & $\begin{array}{l}\text { Pressurized } \\
\text { water }\end{array}$ & 915.0 & 2893.0 & 8006 \\
\hline Seneca, SC & B\&W & $\begin{array}{l}\text { Pressurized } \\
\text { water }\end{array}$ & 860.0 & 2568.0 & 7304 \\
\hline Seneca, SC & B\&W & $\begin{array}{l}\text { Pressurized } \\
\text { water }\end{array}$ & 860.0 & 2568.0 & 7311 \\
\hline Seneca, SC & B\&W & $\begin{array}{l}\text { Pressurized } \\
\text { water }\end{array}$ & 860.0 & 2568.0 & 7409 \\
\hline
\end{tabular}

Full-power license, 8/25/90

Commercial operation, 1/8/90.

water 


\section{A. Central-Station Electric Power Plants (Continued)}

\section{Name (licensee) [docket number]}

OPERABLE (Continued)

Oyster Creek Nuclear Power Plant,

Unit 1 (GPU Nuclear Corp.) [50-219]

Palisades Nuclear Plant, Unit 1

(Consumers Power Co.) [50-255]

Palo Verde Nuclear Generating Station, Unit 1

(Arizona Public Service Co.) [50-528]

Palo Verde Nuclear Generating Station, Unit 2

(Arizona Public Service Co.) [50-529]

Palo Verde Nuclear Genernting Station, Unit 3

(Arizona Public Service Co.) [50-530]

Peach Bottom Atomic Power Station,

Unit 2 (Philadelphia Electric Co.) [50-277]

Peach Bottom Atomic Power Station, Unit 3

(Philadelphia Electric Co.) [50-278]

- Perry Nuclear Power Plant, Unit 1 (Cleveland

Electric Illuminating $\mathrm{Co}$.) [50-440]

Pilgrim Nuclear Power Station, Unit

(Boston Edison Co.) [50-293]

Point Beach Nuclear Plant, Unis 1

(Wisconsin Electric Power Co.) [50-266]

Point Beach Nuclear Plant, Unit 2

(Wisconsin Electric Power Co.) [50-301]

Prairie Island Nuclear Generating Plant, Unit 1

(Northem States Power Co.) [50-282]

Privie Islend Nuclear Generating Plant, Unit 2

(Northem States Power Co.) [50-306]

Quad-Cities Station, Unit 1 (Commonwealth

Edison Co.) [50-254]

Quad-Cities Station, Unit 2 (Commonwealth

Edison Co.) [50-265]

River Bend Station, Unit 1 (Gulf States

Utilities Co.) [50-458]

Robert Emmett Ginna Nuclear Power Plant,

Unit 1 (Rochester Gas \& Electric Corp.)

[50-244]

Salem Nuclear Generating Station, Unit 1

(Public Service Electric \& Gas Co.) [50-272]

Salem Nuclear Generating Station, Unit 2

(Public Service Electric \& Gas Co.) [50-311]

San Onofre Nuclear Generating Station, Unit 1

(Southem Califomia Edison) [50-206]

\begin{tabular}{|c|c|c|c|c|c|}
\hline \multirow[b]{2}{*}{ Location } & \multirow[b]{2}{*}{$\begin{array}{c}\text { Principal } \\
\text { nuclear } \\
\text { contractor }\end{array}$} & \multicolumn{4}{|c|}{ Power } \\
\hline & & Type & $\begin{array}{l}\text { MD capacity } \\
\text { net } M W(e)\end{array}$ & $\begin{array}{l}\text { Licensed } \\
M W(t)\end{array}$ & $\begin{array}{c}\text { Initial } \\
\text { criticality } \\
\text { (yr mo) }\end{array}$ \\
\hline Toms River, NJ & GE & Boiling water & 620.0 & 1930.0 & 6905 \\
\hline South Haven, MI & Comb. & $\begin{array}{l}\text { Pressurized } \\
\text { water }\end{array}$ & 730.0 & 2530.0 & 7105 \\
\hline Wintersburg, AZ & Comb. & $\begin{array}{l}\text { Pressurized } \\
\text { water }\end{array}$ & 1221.0 & 3800.0 & 8505 \\
\hline Wintersburg, $A Z$ & Comb. & $\begin{array}{l}\text { Pressurized } \\
\text { water }\end{array}$ & 1221.0 & 3800.0 & 8604 \\
\hline Wintersburg, AZ & Comb. & $\begin{array}{l}\text { Pressurized } \\
\text { water }\end{array}$ & 1304.0 & 3817.0 & 8710 \\
\hline Lancaster, PA & GE & Boiling water & 1051.0 & 3293.0 & 7309 \\
\hline Lancaster, PA & GE & Boiling water & 1035.0 & 3293.0 & 7408 \\
\hline Perry, OH & GE & Boiling water & 1205.0 & 3579.0 & 8606 \\
\hline Plymouth, MA & GE & Boiling water & 670.0 & 1998.0 & 7206 \\
\hline Two Creeks, WI & West. & $\begin{array}{l}\text { Pressurized } \\
\text { water }\end{array}$ & 485.0 & 1518.0 & 7011 \\
\hline Two Creeks, WI & West. & $\begin{array}{l}\text { Pressurized } \\
\text { water }\end{array}$ & 485.0 & 1518.0 & 7205 \\
\hline Red Wing, $M N$ & West. & $\begin{array}{l}\text { Pressurized } \\
\text { water }\end{array}$ & 503.0 & 1650.0 & 7312 \\
\hline Red Wing, MN & West. & $\begin{array}{l}\text { Pressurized } \\
\text { water }\end{array}$ & 500.0 & 1650.0 & 7412 \\
\hline Cordova, II & $\mathrm{GE}$ & Boiling water & 769.0 & 2511.0 & 7110 \\
\hline Cordova, IL & GE & Boiling water & 769.0 & 2511.0 & 7204 \\
\hline St. Francisville, LA & GE & Boiling water & 936.0 & 2894.0 & 8510 \\
\hline Ontario, NY & West. & $\begin{array}{l}\text { Pressurized } \\
\text { water }\end{array}$ & 470.0 & 1520.0 & 6911 \\
\hline Salem, NJ & West. & $\begin{array}{l}\text { Pressurized } \\
\text { water }\end{array}$ & 1106.0 & 3411.0 & 7612 \\
\hline Salem, NJ & West. & $\begin{array}{l}\text { Pressurized } \\
\text { water }\end{array}$ & 1106.0 & 3411.0 & 8008 \\
\hline San Clemente, CA & West. & $\begin{array}{l}\text { Pressurized } \\
\text { water }\end{array}$ & 436.0 & 1347.0 & 6706 \\
\hline
\end{tabular}


San Onofre Nuclear Generating Station, Unit 2 (Southem Califomia Edison) [50-361]

San Onofre Nuclear Generating Station, Unit 3

(Southem California Edison) [50-362]

Seabrook Nuclear Station, Unit 1 (Public

Service Co. of New Hampshire) [50-443]

Sequoyah Nuclear Plant, Unit 1

(Tennessee Valley Authority) [50-327]

Sequoyah Nuclear Plant, Unit 2

(Tennessee Valley Authority) [50-328]

Shearon Harris Nuclear Powr Plant, Unit

(Carolina Power \& Light Co.) [50-400]

South Texas Project, Unit 1 (Houston

Lighting \& Power Co.) [50-498]

South Texas Project, Unit 2 (Houston

Lighting \& Power Co.) [50-499]

St. Lucie Plant, Unit 1 (Florida

Power \& Light Co.) [50-335]

St. Lucie Plant, Unit 2 (Florida

Power \& Light Co.) [50-389]

Surry Power Station, Unit 1 (Virginia

Electric \& Power Co.) [50-280]

Surry Power Station, Unit 2

(Virginia Electric \& Power Co.) [50-281]

u Susquehanna Steam Electric Station, Unit 1

(Pennsylvania Power \& Light Co.) [50-387]

Susquehanna Steam Electric Station, Unit 2

(Pennsylvania Power \& Light Co.) [50-388]

Three Mile Island Nuclear Station,

Unit 1 (GPU Nuclear Corp.) [50-289]

Trojan Nuclear Plant, Unit 1 (Portland

General Electric Co.) [50-344]

Turkey Point Plant, Unit 3 (Florida Power \&

Light Co.) [50-250]

Turkey Point Plant, Unit 4 (Florida Power \&

Light Co.) [50-251]

Vermont Yankee Nuclear Power Station

(Vermont Yankee Nuclear Power Corp.)

[50-271]

Virgil C. Summer Nuclear Station, Unit 1

(South Carolina Electric \& Gas Co.) [50-395]

Washington Nuclear Project, Unit 2

(Washington Public Power Supply System)

[50-397]

Waterford Generating Station, Unit 3

(Entergy Operations Inc.) [50-382]

William B. McGuire Nuclear Station,

Unit 1 (Duke Power Co.) [50-369]

William B. McGuire Nuclear Station,

Unit 2 (Duke Power Co.) [50-370]
San Clemente, CA

San Clemente, $\mathrm{CA}$

Seabrook, NH

Daisy, TN

Daisy, TN

Bonsal, NC

Bay City, TX

Bay City, TX

Fort Pience, FL

Fort Pierce, FL

Gravel Neck, VA

Gravel Neck, VA

Berwick, PA

Berwick, PA

Middletown, PA

Prescott, OR

Florida City, FL

Florida City, FL

Vemon, VT

Jenkinsville, SC

Richland, WA

Taft, LA

Cowans Ford Dam

NC

Cowans Ford Dam,

NC

Comb.
Comb.

Pressurized

water

West water

Pressurized

water

West. Pressurized

water

West. Pressurized

water

West. Pressurized

water

West. Pressurized

water

West. Pressurized

water

Comb. Pressurized

water

Comb. Pressurized

water

West. Pressurized

water

West. Pressurized

Pressurized

GE Boiling water

GE Boiling water

1070.0

3390.0

8207

$1080.0 \quad 3390.0$

8308

$1198.0 \quad 3411.0$

1148.0

3411.0

8007

$1148.0 \quad 3411.0$

8111

915.0

2775.0

8701

1250.0

3800.0

8803

1250.0

3800.0

8902

839.0

2700.0

7604

839.0

2700.0

8306

$\begin{array}{lll}781.0 & 2441.0 & 7207\end{array}$

781.0

2441.0

7303

$\begin{array}{lll}1032.0 & 3293.0 & 8209\end{array}$

1032.0

3293.0

8405

B\&W Pressurized

water

West. Pressurized

water

West. Pressurized

water

West. Pressurized

water

GE Boiling water

$776.0 \quad 2568.0$

7406

$\begin{array}{lll}1075.0 & 3411.0 & 7512\end{array}$

$\begin{array}{lll}666.0 & 2200.0 \quad 7210\end{array}$

$\begin{array}{lll}666.0 & 2200.0 \quad 7306\end{array}$

$\begin{array}{lll}504.0 & 1593.0 & 7203\end{array}$

West. Pressurized

885.0

2775.0

8210

$1095.0 \quad 3323.0$

8401

Comb. Pressurized

1075.0

3390.0

8503

West. Pressurized

water

West. Pressurized

1150.0

3411.0

8108

water
Low-power license, 5/26/89.

Full-power license, 3/15/90

Commercial Operation, 8/19/90. 


\section{POWER REACTORS}

\section{A. Central-Station Electric Power Plants (Continued)}

Name (licensee) [docket number]

OPERABLE (Continued)

Wolf Creek Generating Station

(Kansas Gas \& Electric Co.) [50-482]

Yankee Nuclear Power Station

(Yankee Atomic Electric Co.) [50-029]

Zion Nuclear Plant, Unit 1

(Commonwealth Edison Co.) [50-295]

Zion Nuclear Plant, Unit 2

(Commonwealth Edison Co.) [50-304]

Name (licensee) [docket number]

\section{BEING BUIL'T}

Bellefonte Nuclear Plant, Unit 1

(Tennessee Valley Authority) [50-438]

Bellefonte Nuclear Plant, Unit 2

(Tennessee Valley Authority) [50-439]

Comanche Peak Steam Electric Station, Unit 2

(Texas Utilities Generating Co.) [50-446]

Perry Nuclear Power Plant, Unit 2 (Cleveland

Electric Illuminating $\mathrm{Co}$.) [50-441]

Washington Nuclear Project, Unit 1

(Washington Public Power Supply System)

[50-460]

Washington Nuclear Projec, Unit 3

(Washington Public Power Supply System)

[50-508]

Watts Bar Nuclear Plant, Unit 1

(Tennessee Valley Authority) [50-390]

Watts Bar Nuclear Plant, Unit 2

(Tennessee Valley Authority) [50-391]

\section{B. Dual-Purpose Plants}

\section{PART I CIVILIAN REACTORS (DOMESTIC)}

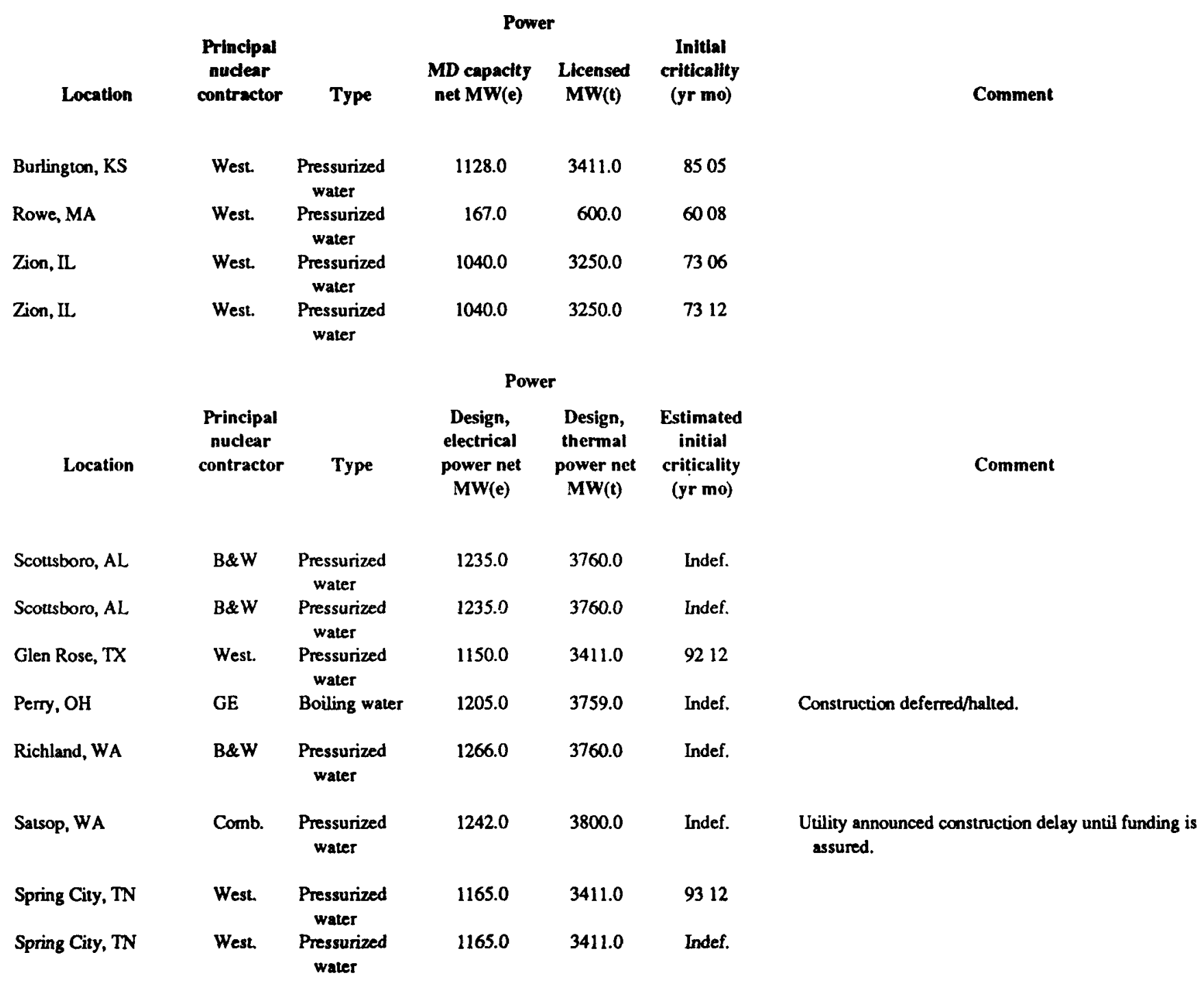




\section{Propulsion (Maritime)}

(No resctors are currently in this category.)

\section{EXPERIMENTAL POWER-REACTOR SYSTEMS}

\section{A. Electric-Power Systems}

\begin{tabular}{|c|c|c|c|c|c|c|c|c|}
\hline \multirow[b]{2}{*}{ Name (Regulatory agency) } & \multicolumn{8}{|c|}{ Power } \\
\hline & Designation & Location & $\begin{array}{l}\text { Principal } \\
\text { nuclear } \\
\text { contractor }\end{array}$ & Type & $\begin{array}{c}\text { Capacity } \\
\text { net } k W(e)\end{array}$ & $\begin{array}{l}\text { Licensed } \\
\text { kW(t) }\end{array}$ & $\begin{array}{c}\text { Initial } \\
\text { criticality } \\
\text { (yr mo) }\end{array}$ & Comment \\
\hline \multicolumn{9}{|l|}{ OPERABLE } \\
\hline Experimental Breeder Reactor II (DOE) & EBR-II & INEL Site, ID & ANL & $\begin{array}{l}\text { Sodium } \\
\text { coled, } \\
\text { fast }\end{array}$ & 20,000 & 62,500 & 6100 & $\begin{array}{l}\text { The EBR-II reactor is a major irradiation facility } \\
\text { for the LMR program; it continues to generate } \\
\text { electric power for the Idaho National Engineering } \\
\text { Laboratory grid. Tests at EBR-II simulating LOF } \\
\text { and LOHS accidents demonstrated that the pool- } \\
\text { type design using metallic fuel will safely shut } \\
\text { itself down without autornatic protection system } \\
\text { or operator action. Advanced metal alloy fuel } \\
\text { subassemblies have achieved bumups in excess } \\
\text { of } 180,000 \mathrm{Mwd} / \mathrm{T} \text {. }\end{array}$ \\
\hline
\end{tabular}

\section{B. Space Nuclear Auxillary Power (SNAP)}

\begin{tabular}{|c|c|c|c|c|c|c|c|c|}
\hline \multirow[b]{2}{*}{ Name (Regulatory agency) } & \multicolumn{7}{|c|}{ Power } & \multirow[b]{2}{*}{ Comment } \\
\hline & Designation & Location & $\begin{array}{l}\text { Principal } \\
\text { nuclear } \\
\text { contractor }\end{array}$ & Type & $\begin{array}{l}\text { Capacity } \\
\text { net kW(e) }\end{array}$ & $\begin{array}{l}\text { Licensed } \\
k W(t)\end{array}$ & $\begin{array}{c}\text { Initial } \\
\text { criticality } \\
\text { (pr mo) }\end{array}$ & \\
\hline \multicolumn{9}{|l|}{ PLANNED } \\
\hline $\begin{array}{l}\text { SP-100 Ground Engineering System Reactor } \\
\text { (DOE) }\end{array}$ & SP-100GES & Hanford Site, WA & WHC & $\begin{array}{l}\text { Thermo- } \\
\text { electric }\end{array}$ & 100 & 2.5 & 9400 & $\begin{array}{l}\text { SP-100 is a nuclear-powered electrical generating } \\
\text { system being developed for use in space, } \\
\text { following extensive ground testing of the reactor } \\
\text { system at Hanford. The facility will have an } \\
\text { innovative design that simulates outer space. } \\
\text { Budget constraints have deferred the ground } \\
\text { reactor testing aspect of the project, but the } \\
\text { research and development is continuing. }\end{array}$ \\
\hline
\end{tabular}

\section{Space Propulsion}

(No reactors are currently in this category.) 


\section{A. General Irradlation Test}

\section{Name (Regulatory agency)}

\section{OPERABLE}

Advanced Test Reacior (DOE)

Fast Flux Test Facility (DOE)

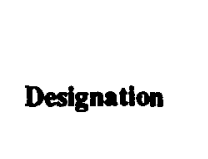

ATR

FFTF

\section{B. High-Power Research and Test}

\section{OPERABLE}

Brookhaven High Flux Beam Research Reactor HFBR (DOE)

$\infty$

Brockhaven Mecical Research Reactor (DOE)

High Flux Isotope Reactor (DOE) HFIR

National Institute of Standards and Technology NIST (NRC)

Omega West Reactor (DOE)

OWR

\section{Safety-Research and Test}

\section{OPERABLE}

Power-Burst Facility (DOE)

Transient Reactor Test (DOE)

INEL, ID

INEL Site, ID

EG\&G-ID

ANL

Open tank

Graphite
28,000

7300 5900

Reactor in standby.

Authorized power, n.a. Transient RX.

\section{General Research}

\section{OPERABLE}

$\begin{array}{lllrrl}\text { Upton, NY } & \text { BNL } & \text { Heavy water } & 30,000 & 6500 & \text { Power derated subject to further safety tests. } \\ & & & 5,000 & 5900 & \\ \text { Upton, NY } & \text { Daystrom } & \text { Tank } & 85,000 & 6500 & \text { Operating. } \\ \text { Oak Ridge, TN } & \text { ORNL } & \text { Tank flux trap } & 20,000 & 6700 & \\ \text { Gaithersburg, MD } & \text { NBS-B\&R } & \text { Heavy water } & & & \\ \text { Los Alamos, NM } & \text { LANL } & \text { Tank } & 8,000 & 5600 & \end{array}$


Aerotest Operations, Inc. (NRC)

Biological Research Reactor (DOE)

Coupled Fast Reactivity Measurement Facility (DOE)

Dow Chemical Co. (NRC)

Fast Source Reactor (DOE)

General Atomics, Advanced TRIGA-Mk F

Prototype Reactor (NRC)

General Atomics, TRIGA-Mk I Prototype

Reactor (NRC)

General Electric Nuclear Test Reactor (NRC)

Neutron Radiography Facility (DOE)

Omaha Veterans Administration Hospital (NRC)

Rhode Island Nuclear Science Center (NRC)

Sandia Pulsed Reactor II (DOE)

Sandia Pulsed Reactor III (DOE)

SNL Annular Core Research Reactor (DOE)

Tower Shielding Reactor No. II (DOE)

U. S. Geological Survey Laboratory

(Department of the Interior) (NRC)

- PLANNEd

Advanced Neutron Source Reactor (DOE)
AGNIR

JANUS

San Ramon, CA

Argonne, IL

INEL Site, ID

Pool-TRIGA core

CFRMF

Midland, MI

TRIGA-Mk I

INEL Site, ID

AFSR

La Jolla, CA

TRIGA-Mk F La Jolla,

TRIGA-Mk I La Jolla, CA

NTR

NRAD

Pleasanton, CA

INEL, ID

TRIGA-Mk I Omaha, NE

RINSC

SPR-II

SPR-III

ACRR

TSR-2

TRIGA-Mk I

Narrangansett, RI

Kirtland AFB

East, NM

Kintand AFB,

East, NM

Kirtland AFB

East, NM

Oak Ridge, TN

Denver, CO

ANS

Oak Ridge, TN
ANL

GA

ANL

Owner

Owner

Tank

U-Zr hydride

Fast

U-Zr hydride

U-Zr hydride

Light water

Pool-TRIGA core

U-Zr hydride

\section{Pool}

Sandia Prompt burst

Sandia

Prompt burst

Sandia

$\mathrm{PoOl}-\mathrm{UO}_{2} \mathrm{BeO}$

core

ORNL Light water

GA

U-Zr hydride

ORNL

$\mathrm{D}_{2} \mathrm{O}$ flux trap
330,000

6500

6400

6800

6700

5900

6000

5800

5700

7700

5900

2,000

6400
6700

6700

7500

7800

6000

6900

\section{E. University Research and Teaching}

\section{Name (Regulatory agency)}

\section{OPERABLE}

Arizona, University of (NRC)

Califomia, Irvine, University of (NRC)

Comell University (NRC)

Comell University Zero Power Reactor (NRC)

Florida, University of (NRC)

Georgia Institute of Technology (NRC)

Idaho State University (NRC)

\begin{tabular}{lll} 
Designation & \multicolumn{1}{c}{ Location } & $\begin{array}{c}\text { Principal } \\
\text { nuclear } \\
\text { contractor }\end{array}$ \\
TRIGA-Mk I & Tucson, AZ & GA \\
TRIGA-Mk I & Irvine, CA & GA \\
TRIGA-Mk II & Ithaca, NY & GA \\
ZPR & Ithaca, NY & Vitro \\
UFTR & Gainesville, FL & GNEC \\
GTRR & Atlanta, GA & GNEC \\
AGN-201P-103 & Pocatello, ID & AGN
\end{tabular}

\section{Authorized Initial \\ power criticality}

Type

U-Zr hydride

U-Zr hydride

U-Zr hydride

Tank

Graphite/water

Heavy water

Homog. solid
In conceptual design.

ast and thermal neutron irradimtions, neutron
ndiography, and thermal and fast fissile assay.

In standby.

Transient.

Transient.

Transient.

Operating.

\section{Comment}

$\begin{array}{rr}100 & 5800 \\ 250 & 6900 \\ 500 & 6200 \\ & 6200 \\ 100 & 5900 \\ 5,000 & 6400 \\ & 6700\end{array}$

Authorized power is negligible.

The AGN-201P-103 was operated at San Ramon, CA, by Aerojet-General Corporation from 1957 to 1966. In 4/67 Idaho State University applied for a license to operate the reactor at Pocatello, ID. Authorized power is negligible. 


\section{E. University Research and Teaching (Continued)}

\section{Name (Regulatory agency)}

OPERABLE (Continued)

Illinois, University of (NRC)

Illinois, University of (NRC)

Iowa State University (NRC)

Kansas State University (NRC)

Lowell, University of (NRC)

Manhattan College (NRC)

Maryland, University of (NRC)

Massachusetts Institute of Technology (NRC)

Michigan, University of (Ford Nuclear Reactor) (NRC)

- Missouri at Rolla, University of (NRC)

Missouri, University of (NRC)

New Mexico, University of (NRC)

Dedgnation Location

\section{Principal \\ nuclear}

contractor

LOPRA

Usana, IL

TRIGA-Mk II Champaign

UTR-10

TRIGA-Mk II

ULR

MCZPR

TRIGA

MITR-II

FNR

UMR-R

MURR

AGN-201M-112 Albuquerque, NM
Urbana, IL

\section{Ames, IA}

Manhattan, KS

Lowell, MA

New York, NY

College Park, MD

Cambridge, MA

Amn Arbor, MI

Rolla, MO

Columbia, MO

GA

GA

AS Inc.

GA

GE

AMP

GA

ACF

B\&W

CW

Owner-IC

AGN

Type

\begin{tabular}{|c|c|}
\hline $\begin{array}{l}\text { Authorized } \\
\text { power } \\
\text { kW(t) }\end{array}$ & $\begin{array}{r}\text { In } \\
\text { critt } \\
\text { (yr }\end{array}$ \\
\hline
\end{tabular}

U-Zr hydride

\section{$\mathrm{U}-\mathrm{Zr}$ hydride}

Graphite/water

U-Zr hydride

Pool

Tank

Tank-TRIGA core

Heavy water

reflected

Pool

Pool

Tank

Homog. solid

$\begin{array}{rr}10 & 7100 \\ 1,500 & 6000 \\ 10 & 5900 \\ 250 & 6200 \\ 1,000 & 7400 \\ & 6400 \\ 250 & 7400 \\ 5,000 & 5800 \\ 2,000 & 5700 \\ & \\ 200 & 6100 \\ 10,000 & 6600 \\ & 6600\end{array}$

North Carolina State University (NRC)

Ohio State University (NRC)

Oregon State University (NRC)

Penn State TRIGA Reactor (NRC)

PULSTAR
OSURR
OSTR
PSTR

Raleigh, NC

Columbus, $\mathrm{OH}$

Corvallis, OR

AMF

University Park,

Lockheed

GA

PSTR

PA

PUR-1

West Lafayette, $\mathbb{N}$

TRIGA-Mk I

Lockheed

Reed College (NRC)

State University of New York (NRC)

Texas A\&M University (NRC)

Texas A\&M University (NRC)

$\begin{array}{lll}\text { AGN-201M-106 } & \text { Buffalo, NY } & \text { AMP } \\ & \text { College Station, TX } & \text { AGN }\end{array}$

TRIGA College Station, TX GA
Pool

Pool

U-Zr hydride

Pool-TRIGA core

1,000

500

1,000

1,000

7200

6100

6700

6500

Pool

U-Zr hydride

Pool

Homog. solid

U-Zr hydride
1
250
2,000
1,000

6200

6800

6100

5700

6100

\section{Comment}

Authorized powwer is negligible.

AGN-201-112 was operated at the Universiry of California, Berkeley, beginning in 1957. The University of New Mexico filed an application in 4/66 for transfer and reconstruction of the reactor at a site on its campus. The reactor achieved criticality at the University of New Mexico on 10/7/66. Authorized power is negligible.

Owner: Pennsylvania State University. From 1955 to 1965 the Penn State reactor was operated as a 200-kW( $(t)$ pool-type reactor fueled with MTRtype elements.

Owner: Buffalo Materials Research Center. Authorized power is negligible.

The Nuclear Science Center Reactor at Texas A\&M University has been modified for 1000-kW steady-state operation with a TRIGA-type core. Power level was $100 \mathrm{~kW}$ prior to modification in 1968. 
Texes a Austin, University of (NRC)

Uth, Univernity of (NRC)

Virginin, Univernity of (NRC)

Washingron State Universiry (NRC)

Wisconsin, Univerity of (NRC)

Worcester Polyrechnic Instituse (NRC)

\section{PLANNED}

Arkansas Tech University (NRC)
TRIGA-Mk II

TRIGA-Mk I

UVAR

WSTR

TRIGA

Madison, WI

Worcester, MA

GE

TRIGA-Mk I

Austin, TX
Sah Lake City, UT
Charlottesville, VA

Pullman, WA

GA

GA
Pool-TRIGA core

1,000

Pool

U-Zr hydride

10

250
$1,100 \quad 8900$

6000

$1,000 \quad 6700$ Washington State University reactor was replaced by a modified TRIGA-type core and control system, and the steady-state power level was increased from 100 to $1000 \mathrm{~kW}(\mathrm{t})$. 6700 The University of Wisconsin reactor has been modified for 1000-kW steady-state operation

with a TRIGA-type core. Power level was $250 \mathrm{~kW}$ prior to modification in 1967.

5900

The Atkansas Tech University reactor contains

parts from the TRIGA reactor dismantled at

Michigan State University.

\section{PART II PRODUCTION REACTORS}

\section{MATERIALS PRODUCTION}

(All owned by DOE)

Destgmation

OPERABLE

C Reactor

K Reactor

L Reactor

P Reactor

PLANNED

Modular High Temperature Gas Reactor

\section{Nuclear}

destgner

Du Pont

Du Pon

Du Pont

Du Pont

INEL
Type

Heavy water

Heavy water

Heavy water

Heavy water

Gas cooled
Location

Start-

up

Aiken, SC

Aiken, SC

Aiken, SC

Aiken, SC

INEL Site, ID
5500

5400

5400

5400

2000
Comment

Cold standby.

In extended outage for maintenance and hardware and software upgrades.

Being defueled for cold standby.

Cold standby.

Four modules of $350 \mathrm{MW}(\mathrm{t})$ each.

\section{PROCESS DEVELOPMENT}

(No reactors in this category.) 


\section{DEFENSE POWER-REACTOR APPLICATIONS}

\section{A. Remote Installations}

(No reactors in this category.)

\section{B. Propulsion (Naval)}

The abbreviations used here are defined as follows:

SSN, Submarine (Nuclear Propulsion)

SSBN, Fleet Ballistic Missile Submarine (Nuclear Propulsion)

CGN, Guided Missile Cruiser (Nuclear Propulsion)

CVN, Aircraft Carrier (Nuclear Propulsion)

Name (all owned by U.S. Navy)

OPERABLE

USS TINOSA

USS JOHN MARSHALL

USS GUARDFISH

USS FLASHER

USS GREENLING

$\sim$ USS GATO

USS ALEXANDER HAMIITON

USS HADDOCK

USS WOODROW WILSON

DANIEL WEBSTER*

USS JAMES MADISON

USS TECUMSEH

USS DANIEL BOONE

USS JOHN C. CALHOUN

USS ULYSSES S. GRANT

USS VON STEUBEN

USS CASIMIR PULASKI

USS STONEWALL JACKSON

SAM RAYBURN

USS STURGEON

USS WHALE

USS TAUTOG

USS BENJAMIN FRANKLIN

USS SIMON BOLIVAR

USS KAMEHAMEHA

USS GEORGE BANCROFT

USS LEWIS AND CLARK

USS JAMES K. POLK

USS GRAYLING

USS POGY

USS ASPRO

\begin{tabular}{llr} 
Designation & \multicolumn{1}{c}{ Shipbuilder } & $\begin{array}{r}\text { Start- } \\
\text { up }\end{array}$ \\
& & \\
SSN606 & Portsmouth & 6300 \\
SSN611 & Newport News & 6200 \\
SSN612 & NYSC & 6600 \\
SSN613 & Electric Boat (Groton) & 6600 \\
SSN614 & GD (Quincy) & 6700 \\
SSN615 & GD (Quincy) & 6700 \\
SSBN617 & Electric Boat (Groton) & 6300 \\
SSN621 & Ingalls & 6700 \\
SSBN624 & Mare Island & 6300 \\
-SSBN626 & Electric Boat (Groton) & 6400 \\
SSBN627 & Newport News & 6400 \\
SSBN628 & Electric Boat (Groton) & 6400 \\
SSBN629 & Mare Island & 6300 \\
SSBN630 & Newpont News & 6400 \\
SSBN631 & Electric Boat (Groton) & 6400 \\
SSBN632 & Newpont News & 6400 \\
SSBN633 & Electric Boat (Groton) & 6400 \\
SSBN634 & Mare Island & 6400 \\
ex-SSBN635 & Newpont News & 6400 \\
SSN637 & Electric Boat (Groton) & 6600 \\
SSN638 & GD (Quincy) & 6800 \\
SSN639 & Ingalls & 6800 \\
SSBN640 & Electric Boat (Groton) & 6500 \\
SSBN641 & Newport News & 6500 \\
SSBN642 & Mare Island & 6500 \\
SSBN643 & Electric Boat (Groton) & 6500 \\
SSBN644 & Newport News & 6500 \\
SSBN645 & Electric Boat (Groton) & 6600 \\
SSN646 & Portsmouth & 6900 \\
SSN647 & NYSC/Ingalls & 7000 \\
SSN648 & Ingalls & 6800 \\
& &
\end{tabular}

Name (all owned by U. S. Navy)

Designation

Shipbuilder

USS PHOEINIX

USS BOSTON

USS BALTIMORE

USS CITY OF CORPUS CHRISTI

USS ALBUQUERQUE

USS PORTSMOUTH

USS MINNEAPOLIS-SAINT PAUL

USS HYMAN G. RICKOVER

USS AUGUSTA

USS SAN FRANCISCO

USS ATLANTA

USS HOUSTON

USS NORFOLK

USS BUFFALO

USS SALT LAKE CTTY

USS OLYMPIA

USS HONOLULU

USS PROVIDENCE

USS PITTSBURGH

USS CHICAGO

USS KEY WEST

USS OKLAHOMA CITY

USS LOUISVILLE

USS HELENA

USS OHIO

USS MICHIGAN

USS FLORIDA

USS GEORGIA

USS HENRY M. JACKSON

USS ALABAMA

USS ALASKA
SSN702

SSN703

SSN704

SSN705

SSN706

SSN707

SSN708

SSN709

SSN710

SSN711

SSN712

SSN713

SSN714

SSN715

SSN716

SSN717

SSN718

SSN719

SSN720

SSN721

SSN722

SSN723

SSN724

SSN725

SSBN726

SSBN727

SSBN728

SSBN729

SSBN730

SSBN731

SSBN732

$\begin{array}{ll}\text { Electric Boat (Groton) } & 8100 \\ \text { Electric Boat (Groton) } & 8100 \\ \text { Electric Boat (Groton) } & 8200 \\ \text { Electric Boat (Groton) } & 8200 \\ \text { Electric Boat (Groton) } & 8200 \\ \text { Electric Boat (Groton) } & 8300 \\ \text { Electric Boat (Groton) } & 8300 \\ \text { Electric Boat (Groton) } & 8400 \\ \text { Electric Boat (Groton) } & 8400 \\ \text { Newport News } & 8000 \\ \text { Newport News } & 8100 \\ \text { Newport News } & 8200 \\ \text { Newport News } & 8300 \\ \text { Newport News } & 8300 \\ \text { Newport News } & 8300 \\ \text { Newport News } & 8400 \\ \text { Newport News } & 8500 \\ \text { Electric Boat (Groton) } & 8500 \\ \text { Electric Boat (Groton) } & 8500 \\ \text { Newport News } & 8600 \\ \text { Newport News } & 8700 \\ \text { Newport News } & 8700 \\ \text { Electric Boat (Groton) } & 8600 \\ \text { Electric Boat (Groton) } & 8700 \\ \text { Electric Boat (Groton) } & 8000 \\ \text { Electric Boat (Groton) } & 8200 \\ \text { Electric Boat (Groton) } & 8200 \\ \text { Electric Boat (Groton) } & 8300 \\ \text { Electric Boat (Groton) } & 8400 \\ \text { Electric Boat (Groton) } & 8400 \\ \text { Electric Boat (Groton) } & 8500\end{array}$

Start.

8300

400

8200

8300

8500

00

00

.

00

$\infty$

800 
USS SUNFISH

USS PARGO

USS PUFFER

USS RAY

USS GEORGE C. MARSHALL

USS HENRY L. STIMSON

USS GEORGE WASHINGTON

CARVER

USS FRANCIS SCOTT KEY

USS MARIANO G. VALLEJO

USS WILL ROGERS

USS SAND LANCE

USS LAPON

USS GURNARD

USS HAMMERHEAD

USS GUITARRO

USS HAWKBILL

USS BERGALL

USS SPADEFISH

USS SEA HORSE

USS FINBACK

USS NARWHAL

USS PINTADO

USS FLYING FISH

USS TREPANG

$\vec{\omega} \quad$ USS BLUEFTSH

USS BILLFISH

USS DRUM

USS ARCHERFISH

USS SILVERSIDES

USS WILLIAM H. BATES

USS BATFISH

USS TUNNY

USS PARCHE

USS CAVALLA

USS L MENDEI L RIVERS

USS RICHARD B. RUSSELL

USS LOS ANGELES

USS BATON ROUGE

USS PHILADELPHIA

USS MEMPHIS

USS OMAHA

USS CINCINNATI

USS GROTON

USS BIRMINGHAM

USS NEW YORK CITY

USS INDIANAPOLIS

USS BREMERTON

USS JACKSONVILLE

USS DALLAS

USS LA JOLLA

SSN649
SSN650
SSN652
SSN653
SSBN654
SSBN655

SSBN656
SSBN657
SSBN658
SSBN659
SSN660
SSN661
SSN662
SSN663
SSN665
SSN666
SSN667
SSN668
SSN669
SSN670
SSN671
SSN672
SSN673
SSN674
SSN675
SSN676
SSN677
SSN678
SSN679
SSN680
SSN681
SSN682
SSN683
SSN684
SSN686
SSN687
SSN688
SSN689
SSN690
SSN691
SSN692
SSN693
SSN694
SSN695
SSN696
SSN697
SSN698
SSN699
SSN700
SSN701

GD (Quincy)

Newport News

Electric Boat (Groton) $\quad 6700$

Portsmouth $\quad 7100$

Newport News $\quad 6700$

Mare Island $\quad 6800$

Newport News $\quad 6700$

Mare Island $\quad 7200$

Mare Island $\quad 7000$

Eloctric Boat (Groton) $\quad 6900$

Newport News $\quad 6900$

Electric Boat (Groton) $\quad 6900$

Newport News $\quad 6900$

Electric Boat (Groton) $\quad 6900$

Mare Island $\quad 7000$

Electric Boat (Groton) $\quad 6900$

Electric Boat (Groton) $\quad 7000$

Electric Boat (Groton) $\quad \mathbf{7 0} 00$

Electric Boat (Groton) $\quad 7000$

Mare Island $\quad 7100$

Electric Boat (Groton) $\quad 7100$

Electric Boat (Groton) $\quad 7100$

7200

Electric Boat (Groton) $\quad 7200$

Ingalls $\quad 7300$

Ingalls

Electric Boat (Groton) $\quad 7200$

Newport News $\quad 7400$

Newport News $\quad 7400$

Newport News $\quad 7600$

Newport News $\quad 7700$

Electric Boat (Groton) $\quad 7600$

Newport News $\quad 7700$

Electric Boat (Groton) $\quad 7700$

Newport News $\quad 7700$

Electric Boat (Groton) $\quad 7700$

Newport News $\quad 7800$

Eloctric Boat (Groton) $\quad 7800$

Electric Boat (Groton) $\quad 7900$

Electric Boat (Groton) 7900

Electric Boat (Groton) $\quad 7900$

Electric Boat (Groton) $\quad 8000$

Electric Boat (Groton) $\quad 8100$
USS NEVADA

USS TENNESSEE

USS PENNSYLVANIA

USS WEST VIRGINIA

USS KENTUCKY

USS MARYLAND

USS NEWPORT NEWS

USS SAN JUAN

USS PASADENA

USS ALBANY

USS TOPEKA

USS MIAMI

USS SCRANTON

USS ALEXANDRIA

USS ASHEVILLE

USS JEFFERSON CITY

USS ANNAPOLIS

USS LONG BEACH (2 reactors)

USS BAINBRIDGE (2 reactors)

USS TRUXTUN (2 reactors)

USS CALIFORNIA (2 reactors)

USS SOUTH CAROLINA

(2 reactors)

USS VIRGINIA (2 reactors)

USS TEXAS (2 reactors)

USS MISSISSIPPI (2 reactors)

USS ARKANSAS (2 reactors)

USS ENTERPRISE (8 reactors)

USS NIMITZ (2 reactors)

USS DWIGHT D.

EISENHOWER (2 reactors)

USS CARL VINSON (2 reactors)

USS THEODORE ROOSEVELT

(2 reactors)

USS ABRAHAM LINCOLN

(2 reactors)

Deep Submergence Research

Vehicle

BEING BUILT

NEBRASKA

RHODE ISLAND

MAINE

Submarine

Submarine

SPRINGFIELD

COLUMBUS

SANTA FE

BOISE

MONTPELIER

CHARLOTTE
SSBN733

SSBN734

SSBN735

SSBN736

SSBN737

SSBN738

SSN750

SSN751

SSN752

SSN753

SSN754

SSN755

SSN756

SSN757

SSN758

SSN759

SSN760

CGN9

CGN25

CGN35

CGN36

CGN37

CGN38

CGN39

CGN40

CGN41

CVN65

CVN68

CVN69

CVN70

CVN71

CVN72

NR-1

SSBN739

SSBN740

SSBN741

SSBN742

SSBN743

SSN761

SSN762

SSN763

SSN764

SSN765

SSN766
Electric Boat (Groton)

Electric Boat (Groton) $\quad 8600$

Electric Boet (Groton)

Electric Bott (Groton) $\quad 8800$

Electric Bont (Groton) $\quad 9000$

Electric Boat (Groton) $\quad 9000$

lectric Boat (Groton)

Newport News $\quad 8800$

Electric Boat (Groton) $\quad 8700$

Electric Boat (Groton) $\quad 8800$

8900

Electric Boat (Groton) $\quad 8900$

Electric Boat (Groton) $\quad 8900$

Newpon News

9100

Newpont News $\quad 9100$

Newport News $\quad 9100$

Electric Boat (Groton) $\quad 9100$

Bethlehem

Bethlehem

NYSC

Newport News

6200

Newport News

Newport News

Newport News

Newpont News

Newport News

Newport News

Newport News

Newport News

Newport News

7300

7400

7600

7700

7800

8000

6000

8100

8600

8900

6900

Electric Boeat (Groton)

Electric Boat (Groton)

Electric Boat (Groton)

Electric Boat (Groton)

Electric Boat (Groton)

Electric Boat (Groton)

Electric Boat (Groton)

Electric Boat (Groton)

Newport News

Newport News

Newport News 


\section{DEFENSE POWER-REACTOR APPLICATIONS}

\section{PART III MILITARY REACTORS}

\section{B. Propulsion (Naval) (Continued)}

\begin{tabular}{|c|c|c|}
\hline Name (all owned by U.S. Navy) & Destgmation & Shipbuilder \\
\hline \multicolumn{3}{|l|}{ BEING BUILT (Continued) } \\
\hline HAMPTON & SSN767 & Newpon News \\
\hline HARTFORD & SSN768 & Electric Boat (Groton) \\
\hline TOLEDO & SSN769 & Newpont News \\
\hline TUCSON & SSN770 & Newpon News \\
\hline COLOMBLA & SSN771 & Electric Boat (Grotion) \\
\hline GREENVILLE & SSN772 & Newport News \\
\hline
\end{tabular}

Start-

up

Name (all owned by U. S. Navy)

Dedgnation

Shipbullder

\section{CHEYENNE}

SEAWOLF

GEORGE WASHINGTON

JOHN C. STENNTS

UNTTED STATES

SSN773

SSN21

CVN73

CVN74

CVN75
Newport News

Electric Boat (Groton)

Newport News

Newport News

Newport News

- Removed from sea-going service and in conversion to training platform (MTS 626).

† Removed from sea-going service and converted to training plaform (MTS 635).

\section{DEVELOPMENTAL POWER}

\section{A. Electric-Power Experiments and Prototypes}

(No reactors in this category.)

\section{B. Propulsion Experiments and Prototypes}

Name (all owned by DOE)

\section{OPERABLE}

Destroyer Renctor Prototype

Large Ship Reactor Prototype (2 reactors)

Modifications and Additions to Reactor Facility

Natural Circulation Test Plant

Small Submarine Reactor Procotype

Trident Prototype

\begin{tabular}{ll} 
Desigmation & \multicolumn{1}{c}{ Location } \\
& \\
D1G & West Milion, NY \\
A1W & INEL Site, ID \\
MARF & West Milion, NY \\
S5G & NEL Site, ID \\
S1C & Windsor, CT \\
S8G & West Milion, NY
\end{tabular}

Principal

contractor

Type

Initial

criticality

(rr mo)

$\begin{array}{lll}\text { GE } & \text { Pressurized water } & 6200 \\ \text { West. } & \text { Pressurized water } & 5800 \\ \text { GE } & \text { Pressurized water } & 7600 \\ \text { West. } & \text { Pressurized water } & 6500 \\ \text { GE } & \text { Pressurized water } & 5900 \\ \text { GE } & \text { Pressurized water } & 7800\end{array}$

\section{TEST AND RESEARCH}

\section{A. Test}

(No reactors in this category.) 


\section{B. Research}

\section{Name (Regulatory agency)}

\section{OPERABLE}

Aberdeen Pulsed Reactor Facilty, Test and

Evaluation Command, USA

Armed Forces Radiobiology Research Instutute

DNA, DOD (NRC)

Fast Burst Reactor Facility, Test and

Evaluation Command, USA

Stationary Neutron Radiography System, USAF

\begin{tabular}{|c|c|c|c|c|c|c|}
\hline Designation & Location & $\begin{array}{l}\text { Principal } \\
\text { nuclear } \\
\text { contractor }\end{array}$ & Type & $\begin{array}{l}\text { Authorized, } \\
k W(t)\end{array}$ & $\begin{array}{c}\text { Initial } \\
\text { criticality } \\
\text { (yr mo) }\end{array}$ & Comment \\
\hline APRF & Aberdeen, MD & UNC & $\begin{array}{l}\text { Bare, fast, } \\
\text { prompt burst }\end{array}$ & 10 & 6800 & \\
\hline AFRRI & Bethesda, MD & GA & TRIGA-Mk F & 1100 & 6200 & \\
\hline FBRF & White Sands, NM & Kaman & $\begin{array}{l}\text { Bare, fast, } \\
\text { prompt burst }\end{array}$ & 10 & 6400 & \\
\hline SNRS-1 & McClellan AFB, CA & GA & TRIGA Mod Mk- $\Pi$ & 1300 & 9001 & Operatung license, $1 / 91$ \\
\hline
\end{tabular}

\section{POWER REACTORS}

\section{A. Central-Station Electric Power Plants}

\section{Reactor name. Owner}

\section{Location}

in OPERABLE

$$
\text { Belgium, Doel, Unit } 1
$$

Belgium, Doel, Unit 2

Antwerp

Belgium, Doel, Unıt 4

Antwerp

Belgıum, Thange, Unit 1

Antwerp

Belgium, Thange, Unit 3

Huy, Luege

Huy, Lege

Brazl, Angra 1, Central Electncia de Fumas

Germany, Mulheum-Kaerhch RhemeschWestfaelısches Elektnzitaetswerk AG

Indıa, Tarapur Nuclear Power Staton,

Unit 1

India, Tarapur Nuclear Power Station,

Unit 2

Italy, Caorso Nuclear Station ENEL

Italy, Tnno Vercellese ENEL

Japan, Fukushima Dai-1chı Power Station, Unit 1 Tokyo Electric Power Co Japan, Fukushıma Da1-1ch Power Stauon, Unit 2 Tokyo Electric Power Co
Angra dos Reis

Mulhem-Kaerhch Bombay)

Tarapur (near Bombay)

Piacenza/

Cremona

Tnno, Predmont

Okuma, Fukushıma Pref

Okuma, Fukushıma Pref
Tarapur (near

NRC
export
license No.
and date

Principal
nuclear
contractor.
Reactor type

Power
design
net

Reactor type

West

Pressunzed wate

West

Pressunzed water

West

Pressunzed water

West /Fram , ACEC

Pressunzed water

West

Pressunzed water

$\mathrm{XR}-081$

West.

04/13/73 Pressunzed water

XR-118

06/28/77 Pressunzed water

XR-054 GE

07/07/64 Bollung water

XR-054

07/07/64

XR-077

04/02/1

$\mathrm{XR}-044$

$06 / 14 / 62$

XR-066

08/15/67

XR-072

$04 / 22 / 70$
GE

Bolling water

GE

Bollng water

Pressunzed water

GE

Bollung water

GE, Toshiba

Bollung water
PART IV EXPORT REACTORS

Comment

$\begin{array}{rrrr}3920 & 11920 & 00 & 7500 \\ 3920 & 11920 & 00 & 7500 \\ 10060 & 30000 & 00 & 8500 \\ 8700 & 26600 & 00 & 7500 \\ 10060 & 30000 & 00 & 8500 \\ 6260 & 18820 & 00 & 8500 \\ 12000 & 00 & 00 & 8710 \\ 2000 & 7070 & 00 & 6900 \\ 2000 & 7070 & 00 & 6900 \\ 8400 & 26510 & 00 & 7900 \\ 2470 & 8700 & 00 & 6500 \\ 4390 & 13800 & 00 & 7010 \\ 7600 & 23810 & 00 & 7305\end{array}$




\section{POWER REACTORS}

PART IV EXPORT REACTORS

\section{A. Central-Station Electric Power Plants (Continued)}

\begin{tabular}{|c|c|c|c|c|c|c|c|c|}
\hline Reactor name. Owner & Location & $\begin{array}{c}\text { NRC } \\
\text { export } \\
\text { license No. } \\
\text { and date }\end{array}$ & $\begin{array}{l}\text { Principal } \\
\text { nuclear } \\
\text { contractor. } \\
\text { Reactor type }\end{array}$ & $\begin{array}{c}\text { Power } \\
\text { design } \\
\text { net } \\
\text { MW(e) }\end{array}$ & $\begin{array}{l}\text { Power } \\
\text { MW(t) }\end{array}$ & $\begin{array}{l}\text { Author- } \\
\text { ized } \\
\text { power } \\
\text { kW(t) }\end{array}$ & $\begin{array}{l}\text { Initial } \\
\text { criti- } \\
\text { cality } \\
\text { (yr mo) }\end{array}$ & Comment \\
\hline \multicolumn{9}{|l|}{ OPERABLE (Continued) } \\
\hline $\begin{array}{l}\text { Japan, Fukushima Dai-ichi Power Station, } \\
\text { Unit 6. Tokyo Electric Power Co. }\end{array}$ & $\begin{array}{l}\text { Futaba, Fukushima } \\
\text { Pref. }\end{array}$ & $\begin{array}{l}\text { XR-084 } \\
05 / 25 / 73\end{array}$ & $\begin{array}{l}\text { GE, Toshiba. } \\
\text { Boiling water }\end{array}$ & 1067.0 & 3293.0 & 0.0 & 7903 & \\
\hline $\begin{array}{l}\text { Japan, Mihama Power Station, Unit } 1 . \\
\text { Kansai Electric Power Co. }\end{array}$ & $\begin{array}{l}\text { Mihama, Fukui } \\
\text { Pref. }\end{array}$ & $\begin{array}{l}\text { XR-067 } \\
08 / 15 / 67\end{array}$ & $\begin{array}{l}\text { West., Mitsubishi. } \\
\text { Pressurized water }\end{array}$ & 320.0 & 1031.0 & 0.0 & 7007 & \\
\hline $\begin{array}{l}\text { Japan, Ohi Power Station, Unit 1. Kansai } \\
\text { Electric Power Co. }\end{array}$ & Ohi, Fukui Pref. & $\begin{array}{l}\text { XR-082 } \\
04 / 17 / 73\end{array}$ & $\begin{array}{l}\text { West., Mitsubishi. } \\
\text { Pressurized water }\end{array}$ & 1120.0 & 3423.0 & 0.0 & 7712 & \\
\hline $\begin{array}{l}\text { Japan, Ohi Power Station, Unit 2. Kansai } \\
\text { Electric Power Co. }\end{array}$ & Ohi, Fukui Pref. & $\begin{array}{l}\text { XR-082 } \\
04 / 17 / 73\end{array}$ & $\begin{array}{l}\text { West., Mitsubishi. } \\
\text { Pressurized water }\end{array}$ & 1120.0 & 3423.0 & 0.0 & 7809 & \\
\hline $\begin{array}{l}\text { Japan, Takahama Power Station, Unit } 1 . \\
\text { Kansai Electric Power Co. }\end{array}$ & $\begin{array}{l}\text { Takahame, } \\
\text { FukuiPref. }\end{array}$ & $\begin{array}{l}\text { XR-079 } \\
07 / 23 / 11\end{array}$ & $\begin{array}{l}\text { West., Mitsubishi. } \\
\text { Pressurized water }\end{array}$ & 780.0 & 2440.0 & 0.0 & 7403 & \\
\hline $\begin{array}{l}\text { Japan, Tokai No. } 2 \text { Power Station. Japan } \\
\text { Atomic Power Co. }\end{array}$ & $\begin{array}{l}\text { Tokai-Mura, } \\
\text { Ibaraki Pref. }\end{array}$ & $\begin{array}{l}\text { XR-085 } \\
05 / 25 / 73\end{array}$ & $\begin{array}{l}\text { GE, Hitachi, Shimizu. } \\
\text { Boiling water }\end{array}$ & 1080.0 & 3293.0 & 0.0 & 7801 & \\
\hline $\begin{array}{l}\text { Japan, Tsuruga Power Station, Unit 1. Japan } \\
\text { Alomic Power Co. }\end{array}$ & $\begin{array}{l}\text { Tsuruga, Fukui } \\
\text { Pref. }\end{array}$ & $\begin{array}{l}X R-065 \\
06 / 22 / 67\end{array}$ & $\begin{array}{l}\text { GE. } \\
\text { Boiling water }\end{array}$ & 341.0 & 1064.0 & 0.0 & 6910 & \\
\hline Korea, Kori-1. Korea Electric Power Co. & $\begin{array}{l}\text { Kori (near } \\
\text { Pusan) }\end{array}$ & $\begin{array}{l}\text { XR-083 } \\
05 / 04 / 73\end{array}$ & $\begin{array}{l}\text { West. } \\
\text { Pressurized water }\end{array}$ & 564.0 & 1729.0 & 0.0 & 7800 & Formerly, Korea, Unit 1 \\
\hline Korea, Kori-2. Korea Electric Power Co. & $\begin{array}{l}\text { Kori (near } \\
\text { Pusan) }\end{array}$ & $\begin{array}{l}\text { XR-119 } \\
04 / 08 / 77\end{array}$ & $\begin{array}{l}\text { West. } \\
\text { Pressurized water }\end{array}$ & 605.0 & 1876.0 & 0.0 & 8300 & Formerly, Korea, Unit 2 \\
\hline Korea, Kori-3. Kores Electric Power Co. & Kori (near Pusan) & $\begin{array}{l}\text { XR-131 } \\
10 / 04 / 78\end{array}$ & $\begin{array}{l}\text { West. } \\
\text { Pressurized water }\end{array}$ & 900.0 & 2775.0 & 0.0 & 8500 & Formerly, Korea, Unit 5 \\
\hline Korea, Kori-4. Korea Electric Power Co. & Kori (near Pusan) & $\begin{array}{l}\text { XR-131 } \\
10 / 04 / 78\end{array}$ & $\begin{array}{l}\text { West. } \\
\text { Pressurized water }\end{array}$ & 900.0 & 2775.0 & 0.0 & 8500 & Formerly, Korea, Unit 6 \\
\hline $\begin{array}{l}\text { Korea, Yonggwang-1. Korea Electric } \\
\text { Power Co. }\end{array}$ & $\begin{array}{l}\text { Gyema (near } \\
\text { Kwang Ju) }\end{array}$ & $\begin{array}{l}\text { XR-133 } \\
09 / 22 / 80\end{array}$ & $\begin{array}{l}\text { West. } \\
\text { Pressurized water }\end{array}$ & 900.0 & 2775.0 & 0.0 & 8600 & Formerly, Korea, Unit 7 \\
\hline $\begin{array}{l}\text { Kores, Yonggwang-2. Korea Electric } \\
\text { Power Co. }\end{array}$ & $\begin{array}{l}\text { Gyema (near } \\
\text { Kwang Ju) }\end{array}$ & $\begin{array}{l}X R-133 \\
09 / 22 / 80\end{array}$ & $\begin{array}{l}\text { West. } \\
\text { Pressurized water }\end{array}$ & 900.0 & 2775.0 & 0.0 & 8700 & Formerly, Korea, Unit 8 \\
\hline Mexico, Laguna Verde Station, Unit 1. & Laguna Verde & $\begin{array}{l}\text { XR-098 } \\
05 / 17 / 74\end{array}$ & $\begin{array}{l}\text { GE. } \\
\text { Boiling water }\end{array}$ & 654.0 & 1931.0 & 0.0 & 8811 & Commercial operation, 7/29/90. \\
\hline Netheriands, Dodewaard. GKN & $\begin{array}{l}\text { Dodewaard, } \\
\text { Betuwe }\end{array}$ & $\begin{array}{l}\text { XR-058 } \\
09 / 15 / 65\end{array}$ & $\begin{array}{l}\text { GE. } \\
\text { Boiling water }\end{array}$ & 55.0 & 183.0 & 0.0 & 6806 & $\begin{array}{l}\text { In } 1984 \text { the reactor's nominal power was raised from } \\
163.4 \mathrm{MW}(\mathrm{t}) \text { to } 183 \mathrm{MW}(\mathrm{t}) \text {. The reactor's cooling is } \\
\text { by natural circulation only. }\end{array}$ \\
\hline $\begin{array}{l}\text { Spain, Almaraz, Unit 1. Union } \\
\text { Electrica, S.A. }\end{array}$ & Almaraz & $\begin{array}{l}\text { XR-088 } \\
07 / 12 / 73\end{array}$ & $\begin{array}{l}\text { West. } \\
\text { Pressurized water }\end{array}$ & 902.0 & 2696.0 & 0.0 & 8100 & \\
\hline $\begin{array}{l}\text { Spain, Almaraz, Unit 2. Union } \\
\text { Electrica, S.A. }\end{array}$ & Almaraz & $\begin{array}{l}\text { XR-088 } \\
07 / 12 / 73\end{array}$ & $\begin{array}{l}\text { West. } \\
\text { Pressurized water }\end{array}$ & 902.0 & 2696.0 & 0.0 & 8300 & \\
\hline Spain, Asco, Unit 1. FECSA & Asco & $\begin{array}{l}\text { XR-090 } \\
07 / 12 / 73\end{array}$ & $\begin{array}{l}\text { West. } \\
\text { Pressurized water }\end{array}$ & 902.0 & 2696.0 & 0.0 & 8300 & \\
\hline Spain, Asco, Unit 2. FECSA & Asco & $\begin{array}{l}\text { XR-099 } \\
06 / 22 / 76\end{array}$ & $\begin{array}{l}\text { West. } \\
\text { Pressurized water }\end{array}$ & 902.0 & 2696.0 & 0.0 & 8500 & \\
\hline $\begin{array}{l}\text { Spain, Cofrentes, Unit 1. Hidroelectrica } \\
\text { Espanola S.A. }\end{array}$ & Cofrentes & $\begin{array}{l}\text { XR-097 } \\
06 / 10 / 74\end{array}$ & $\begin{array}{l}\text { GE. } \\
\text { Boiling water }\end{array}$ & 975.0 & 2900.0 & 0.0 & 8400 & \\
\hline Spain, Jose Cabrera. Union Electrica, S.A. & $\begin{array}{l}\text { Zorita de los } \\
\text { Canes }\end{array}$ & $\begin{array}{l}\text { XR-059 } \\
10 / 22 / 65\end{array}$ & $\begin{array}{l}\text { West } \\
\text { Pressurized water }\end{array}$ & 160.0 & 510.0 & 0.0 & 6900 & \\
\hline
\end{tabular}


Spain, Santa Maria de Garofia. Centrales Nucleares del Norte, S.A., Nuclenor

Spain, Vandellos, Unit 2. ENHER

Sweden, Ringhals, Unit 2. Swedish State Power Board

Sweden, Ringhals, Unit 3. Swedish State Power Boand

Sweden, Runghals, Untt 4. Swedish State

Power Boand

Switzerland, Beznau, Unit 1.

Nordostschwe1zerische Kraftwerke AG

Swizzerland, Beznau, Unit 2.

Nordostschweizensche Kraftwerke AG

Switzerland, Leibstadt. Kernkrafiwerk

Leibstadt

Swizzerland, Muhleberg. Bemische

Kraftwerke AG

Tawwan, Chinshan, Unit 1. Tawan

Power Co.

Tawwan, Chinshan, Unit 2. Talwan

Power Co.

Tawan, Kuo Sheng, Unut 1. Tawan

Power Co.

Tawan, Kuo Sheng, Unt 2. Tawan

Power Co.

Tawan, Maanshan, Unt 1. Tawwan Power Co

Tawan, Maanshan, Unt 2. Tawan

Power Co

Yugoslavia, Krsko. Nukleama Elektrama Krsko

\section{BEING BUILT}

England, Sizewell B. Central Electncity Generatung Board

Japan, Kashiwazaka-Kanwa, Unit 6. Tokyo

Electnc Power Co.

Japan, Kashıwazaka-Kanwa, Unıt 7. Tokyo Electnc Power Co.

\section{Korea, Yonggwang-3. Korea Electnc}

Power Co.

Korea, Yonggwang-4. Korea Electnc

Power Co.

Mexico, Laguna Verde Station, Unit 2.

Phuluppines, Republic of the Phulippine Nuclear Power Plant, Unut 1

Span, Lemonz, Unit 1

\begin{tabular}{|c|c|c|c|c|c|c|c|}
\hline $\begin{array}{l}\text { S.M. Garoña } \\
\text { Burgos }\end{array}$ & $\begin{array}{l}\text { XR-064 } \\
06 / 09 / 67\end{array}$ & $\begin{array}{l}\text { GE. } \\
\text { Bolling water }\end{array}$ & $\mathbf{4 4 0 . 0}$ & 1381.0 & 0.0 & 7000 & \\
\hline Tarragona & $\begin{array}{l}\text { XR-122 } \\
09 / 13 / 87\end{array}$ & $\begin{array}{l}\text { West. } \\
\text { Pressurized water }\end{array}$ & 920.0 & 2785.0 & 0.0 & 8803 & \\
\hline $\begin{array}{l}\text { Vārö (near } \\
\text { Göteborg) }\end{array}$ & $\begin{array}{l}\text { XR-069 } \\
05 / 09 / 69\end{array}$ & $\begin{array}{l}\text { West. } \\
\text { Pressurized water }\end{array}$ & 870.0 & 2660.0 & 0.0 & 7410 & \\
\hline $\begin{array}{l}\text { Värö (near } \\
\text { Göteborg) }\end{array}$ & $\begin{array}{l}\text { XR-095 } \\
02 / 02 / 74\end{array}$ & $\begin{array}{l}\text { West. } \\
\text { Pressurized water }\end{array}$ & 915.0 & 2783.0 & 0.0 & 8100 & \\
\hline $\begin{array}{l}\text { Varö (near } \\
\text { Göreborg) }\end{array}$ & $\begin{array}{l}\text { XR-103 } \\
10 / 21 / 75\end{array}$ & $\begin{array}{l}\text { West. } \\
\text { Pressurized water }\end{array}$ & 915.0 & 2783.0 & 0.0 & 8300 & \\
\hline Dörtungen & $\begin{array}{l}\text { XR-063 } \\
02 / 03 / 67\end{array}$ & $\begin{array}{l}\text { West. } \\
\text { Pressurized water }\end{array}$ & 350.0 & 1130.0 & 0.0 & 6900 & \\
\hline Dótungen & $\begin{array}{l}\text { XR-070 } \\
11 / 05 / 69\end{array}$ & $\begin{array}{l}\text { West. } \\
\text { Pressunzed water }\end{array}$ & 350.0 & 1130.0 & 0.0 & 7100 & \\
\hline Leibstadi & $\begin{array}{l}\text { XR-104 } \\
12 / 31 / 75\end{array}$ & $\begin{array}{l}\text { GE. } \\
\text { Bolling water }\end{array}$ & 990.0 & 3138.0 & 0.0 & 8400 & \\
\hline $\begin{array}{l}\text { Muihleberg (near } \\
\text { Bem) }\end{array}$ & $\begin{array}{l}\text { XR-068 } \\
10 / 04 / 67\end{array}$ & $\begin{array}{l}\text { GE. } \\
\text { Bollung water }\end{array}$ & 320.0 & 997.0 & 0.0 & 7200 & \\
\hline Shihmen & $\begin{array}{l}\text { XR-080 } \\
07 / 24 / 72\end{array}$ & $\begin{array}{l}\text { GE. } \\
\text { Boiling water }\end{array}$ & 604.0 & 1775.0 & 0.0 & 7710 & \\
\hline Shahmen & $\begin{array}{l}\text { XR-080 } \\
07 / 24 / 72\end{array}$ & $\begin{array}{l}\text { GE. } \\
\text { Bollng water }\end{array}$ & 604.0 & 1775.0 & 0.0 & 7811 & \\
\hline Wanlh Hsiang & $\begin{array}{l}\text { XR-096 } \\
04 / 17 / 74\end{array}$ & $\begin{array}{l}\text { GE. } \\
\text { Bolling water }\end{array}$ & 948.0 & 2894.0 & 0.0 & 8102 & \\
\hline Wanli Hsıang & $\begin{array}{l}\text { XR-096 } \\
04 / 17 / 74\end{array}$ & $\begin{array}{l}\text { GE. } \\
\text { Bouling water }\end{array}$ & 948.0 & 2894.0 & 0.0 & 8203 & \\
\hline Heng-chun & $\begin{array}{l}\text { XR-113 } \\
06 / 08 / 79\end{array}$ & $\begin{array}{l}\text { West. } \\
\text { Pressunzed water }\end{array}$ & 890.0 & 2785.0 & 0.0 & 8403 & \\
\hline Heng-chun & $\begin{array}{l}\text { XR-113 } \\
06 / 08 / 79\end{array}$ & $\begin{array}{l}\text { West. } \\
\text { Pressunzed water }\end{array}$ & 890.0 & 2785.0 & 00 & 8502 & \\
\hline Krsko & $\begin{array}{l}\text { XR-107 } \\
05 / 20 / 77\end{array}$ & $\begin{array}{l}\text { West. } \\
\text { Pressunzed water }\end{array}$ & 615.0 & 1882.0 & 00 & 8300 & \\
\hline Suffolk & $\begin{array}{l}\text { XR-148 } \\
07 / 30 / 86\end{array}$ & $\begin{array}{l}\text { West. } \\
\text { Pressunzed water }\end{array}$ & 1150.0 & 3425.0 & 0.0 & 9400 & \\
\hline $\begin{array}{l}\text { Kashiwazakı, } \\
\text { Nugata Pref. }\end{array}$ & & $\begin{array}{l}\text { GE., Toshibe. } \\
\text { Advanced boiling } \\
\text { water }\end{array}$ & 1356.0 & 3930.0 & 0.0 & 9600 & Gross design power. \\
\hline $\begin{array}{l}\text { Kashiwazak1, } \\
\text { Nugata Pref. }\end{array}$ & & $\begin{array}{l}\text { GE, Hitachi. } \\
\text { Advanced boiling } \\
\text { water }\end{array}$ & 1356.0 & 3930.0 & 0.0 & 9700 & Gross design power. \\
\hline $\begin{array}{c}\text { Gyema (near } \\
\text { Kwang Ju) }\end{array}$ & $\begin{array}{l}\text { XR-150 } \\
04 / 16 / 87\end{array}$ & $\begin{array}{l}\text { Comb. } \\
\text { Pressunzed water }\end{array}$ & 900.0 & 0.0 & 0.0 & 9500 & Also, Korea, Unit 11. \\
\hline $\begin{array}{c}\text { Gyema (near } \\
\text { Kwang Ju) }\end{array}$ & $\begin{array}{l}\text { XR-150 } \\
04 / 16 / 87\end{array}$ & $\begin{array}{l}\text { Comb. } \\
\text { Pressunzed water }\end{array}$ & 900.0 & 0.0 & 0.0 & 9600 & Also, Korea, Unit 12. \\
\hline Laguna Verde & $\begin{array}{l}\text { XR-102 } \\
10 / 24 / 74\end{array}$ & $\begin{array}{l}\text { GE } \\
\text { Bollung water }\end{array}$ & 654.0 & 1931.0 & 0.0 & 9312 & \\
\hline $\begin{array}{l}\text { Morong, Bataan } \\
\text { prov., Luzon }\end{array}$ & $\begin{array}{l}\text { XR-120 } \\
05 / 06 / 80\end{array}$ & $\begin{array}{l}\text { West. } \\
\text { Pressunzed water }\end{array}$ & 620.0 & 1876.0 & 0.0 & Indef. & \\
\hline Lemonz & $\begin{array}{l}\text { XR-089 } \\
07 / 12 / 73\end{array}$ & $\begin{array}{l}\text { West. } \\
\text { Pressunzed water }\end{array}$ & 900.0 & 2696.0 & 0.0 & Indef. & $97 \%$ complete. \\
\hline
\end{tabular}


A. Central-Station Electric Power Plants (Continued)

\begin{tabular}{|c|c|c|c|c|c|c|}
\hline Reactor nome. Owner & Location & $\begin{array}{c}\text { NRC } \\
\text { export } \\
\text { license No. } \\
\text { and date }\end{array}$ & $\begin{array}{l}\text { Princlpal } \\
\text { nuclear } \\
\text { contractor. } \\
\text { Reactor type }\end{array}$ & $\begin{array}{c}\text { Power } \\
\text { destgn } \\
\text { net } \\
\text { MW(e) }\end{array}$ & $\begin{array}{l}\text { Power } \\
\text { MW(t) }\end{array}$ & $\begin{array}{c}\text { Author- } \\
\text { lzed } \\
\text { power } \\
\text { kW(t) }\end{array}$ \\
\hline
\end{tabular}

\section{BEING BUILT (Continued)}

Spain, Lemoniz, Unit 2

Spain, Valdecaballeros, Unit 1. HE: Sevillana de Elecaricidad

Spain, Valdecaballeros, Unit 2. HE:

Sevillana de Electricidad

\section{PLANNED}

Kores, Ulchin 3. Kores Elearic Power Co.

Korea, Ulchin 4. Korea Elearic Power Co.

Taiwen, Unit 7. Taiwan Power Co.

Lemoniz
Valdecaballero,
Badajos
Valdecaballero,
Badajos

Kyongsengbuk-do

Yenliao
$X R-089$

$07 / 12 \pi 3$

$\mathrm{XR}-110$

05/05/77

$\mathrm{XR}-110$

$05 / 05 / 77$

Kyongsangbuk-do

Yenliao

\section{XR-53}

Pending

XR-53

Pending

$02 / 17 / 81$

\section{West.}

Pressurized water

GE.

Boiling water

GE.

Boiling water

\section{Comb.}

Pressurized water

Comb.

Pressurized wate
900.

975.0

975.0

2696.0

2894.0

2894.0

950

2825

950

1000.0

1000.0
0.0

$$
0.0
$$

0.0

9901

\section{B. Propulsion}

(No resciors in this category)

\section{TEST, RESEARCH, AND TEACHING}

\section{A. General Irradiation Test}

\section{Reactor name. Owner}

\section{OPERABLE}

Japan, JRR-2. Japan Atomic Energy

Research Institute

Japan, NSRR. Japan Atomic Energy

Research Institute

Netherlands. Energy Center

$$
\text { NRC }
$$

export license No.

and date

\section{XR-015}

10/11/57

XR-101

10/16/74

XR-017

$01 / 17 / 58$

XR-091

$06 / 29 / 73$

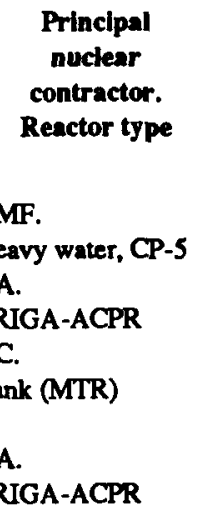

Power

design

net

$\mathbf{M W ( e )}$

Power

Author

Initial

lzed critt-

power cality

kW(t) (yr mo)

Comment

$\begin{array}{llll}0.0 & 0.0 & 10,000.0 & 6010\end{array}$

0.0

0.0

300.0

7506

0.0

0.0

$45,000.0$

6109

0.0

0.0

500.0
Export license application dated 8/31/90.

Export license application dared 8/31/90.

The NRC has issued three licenses to vendors for this reactor: XR-134, -135, and -136. Only the vendor whe wins the contract will be allowed to use its license. The NRC has issued three licenses to vendors for this reactor: XR-134, -135 , and -136 . Only the vendor who wins the contract will be allowed to use its license.

1985 the reactor vessel was replaced. It is now refurbished. Manufacturer of the vessel: Royal Schelde of Flushing (Vlissingen, Holland). 
Romania. Instinute for Nuclear

Technologies

South Africa, Sefari-1. Atomic Energy

Board

Sweden. Sudsvik Energiteknik

\section{B. General Research}

\section{OPERABLE}

Australia, Moata. Atomic Energy

Commission

Austria, Astra. Seibersdorf Research Center

Bangladesh. Institure of Nuclear Technology

Colombia, IAN-R1. Instinue of Nuclear Affairs

Denmark, DR-1. Riső National

Laboratory

England. Imperial Chemical Industries

Greece, Democritos. Atomic Energy

Commission

Indonesin. National Atomic Energy Agency

Indonesia. National Atomic Energy Agency

Bucharest

$X R-091$

$06 / 29 / 73$

Pelindabe (near

XR-042

Pretoria)

$06 / 14 / 61$

Studsvik

XR-019

05/14/58

AC.

Tank (MTR)

\begin{tabular}{|c|c|c|}
\hline $\begin{array}{l}\text { Lucas Heights, } \\
\text { New South } \\
\text { Wales }\end{array}$ & $\begin{array}{l}\text { XR-039 } \\
09 / 12 / 60\end{array}$ & $\begin{array}{l}\text { AR. } \\
\text { UTR-10 }\end{array}$ \\
\hline Seibersdorf & $\begin{array}{l}\text { XR-023 } \\
09 / 03 / 58\end{array}$ & $\begin{array}{l}\text { AMF. } \\
\text { Pool }\end{array}$ \\
\hline Dhaka & $\begin{array}{l}\text { XR-126 } \\
10 / 05 / 82\end{array}$ & $\begin{array}{l}\text { GA. } \\
\text { TRIGA-Mk I }\end{array}$ \\
\hline Bogots & $\begin{array}{l}\text { XR-053 } \\
05 / 27 / 64\end{array}$ & $\begin{array}{l}\text { Lockheed. } \\
\text { Pool }\end{array}$ \\
\hline Risö & $\begin{array}{l}\text { XR-005 } \\
04 / 04 / 57\end{array}$ & $\begin{array}{l}\text { AI. } \\
\text { L-55 }\end{array}$ \\
\hline $\begin{array}{l}\text { Billingham, } \\
\text { Teesside }\end{array}$ & $\begin{array}{l}\text { XR-074 } \\
03 / 23 / 71\end{array}$ & $\begin{array}{l}\text { GA. } \\
\text { TRIGA-Mk I }\end{array}$ \\
\hline Athens & $\begin{array}{l}\text { XR-014 } \\
09 / 25 / 57\end{array}$ & $\begin{array}{l}\text { AMF. } \\
\text { Pool }\end{array}$ \\
\hline Bandung & XR-048 & $\begin{array}{l}\text { GA. } \\
\text { TRIGA-Mk In }\end{array}$ \\
\hline Yogyakarta & & $\begin{array}{l}\text { GA. } \\
\text { TRIGA-Mk II }\end{array}$ \\
\hline
\end{tabular}

GA.

TRIGA (MPR 16)

AC.

ank

Nahal Soreq
Rome
Kingston
Seoul
Seoul
Kuala Lumpur
Salazar

$\begin{array}{llll}0.0 & 0.0 & 14,000.0 & 7900 \\ 0.0 & 0.0 & 20,000.0 & 6500 \\ 0.0 & 0.0 & 50,000.0 & 6000\end{array}$

0.0

0.0

15.0

6100

$\begin{array}{llll}0.0 & 0.0 & 5,000.0 & 6000\end{array}$

0.0

0.0

0.0

0.0

0.0

0.0

0.0

0.0
$\mathrm{XR}-021$

$06 / 12 / 58$

$\mathrm{XR}-026$

X1/08/59

XR-094

$06 / 03 / 75$

XR-027

$05 / 21 / 59$

XR-073

05/15/70

$\mathrm{XR}-125$

02/20/81

$\mathrm{XR}-057$

$02 / 12 / 65$
AMF.

Pool

TRIGA-Mk II

Research reactor

GA.

TRIGA-Mk II

GA.

TRIGA-Mk III

TRIGA-Mk II

GA.

TRIGA-Mk III
Additional NRC export license No. and date: XR-078, $5 / 20 / 71$.

This reactor was designed and built by BATAN

(National Atomic Energy Agency of Indonesia). The design was based on the design of TRIGA Mark II reactor, with maximum power level of $250 \mathrm{~kW}$. In 1979 this reactor reached initial criticality at $50 \mathrm{~kW}$. After the upgrading and the replacing of some

components, it reached a power level of $100 \mathrm{~kW}$ in 1984.

Mexico. National Commission for Nuclear

Finerev 


\section{B. General Research (Continued)}

\section{Reactor name. Owner}

OPERABLE (Continued)

Philippines, Republic of the, PRR-1.

Philippine Atomic Energy Commission

Pontugal, RP-1. National Laboratory of

Engineering and Industrial Technology

Spain. Nuclear Energy Board-JEN

Switzerland. Paul Scherrer Institute

Thailand, TRR-1. Office of Atomic Energy for Peace

\section{Turkey. Atomic Energy Commission}

Turkey. Technical University of Istanbul

Venezuela. Institute for Scientific

Research

Yugoslavia. Josef Stefan Nuclear Institute

Zaire. Regional Center for Nuclear Studies

BEING BUILT

Morocco. C.E.N., Maâmora

PLANNED

Albania
Location

Quezon City

NRC
export
license No.
and date

Principal
nuclear
contractor

Reactor type

XR-034

$11 / 16 / 59$

GA.

TRIGA Conversion

0.0

Power

net

0

0.0

$3,000.0$

8803

Sacavém

Madrid

Wuerenlingen

Bangkok

XR-013

$09 / 13 / 57$

$\mathrm{XR}-010$

$07 / 29 / 57$

AMF.
Pool

GE.

Pool

ORNL.

Pool Conversion

$\mathrm{XR}-112$

GA.

05/05/77 TRIGA-Mk III

0.0
0.
0.0

0.0
Istanbul

XR-030

09/04/59

$\mathrm{XR}-108$

$03 / 24 / 76$

XR-018

$01 / 16 / 58$

XR-055

01/30/64

Kinshasa

\section{AMF.}

Pool

GA.

TRIGA-Mk II

GE.

Pool

GA.

TRIGA-Mk II

GA.

Rabat

XR-158

10/25/91

GA.

TRIGA-Mk II

0.0

0.0

XR-154 GA.

Pending TRIGA-Mk I
Tirana
$1,500.0$

\section{Comment}

The original Philippine Research Reactor (PRR-1) was designed and built by GE andwas commissioned as a $1 \mathrm{MW}$ reactor in 1963 . The reactor was shut down 1/85 for extensive upgrading and has now become a TRIGA Conversion. It has a power level of $3 \mathrm{MW}$ and reached criticality on $3 / 11 / 88$.

$\begin{array}{rr}1,000.0 & 6100 \\ 3,000.0 & 5800 \\ 10,000.0 & 5700 \\ 2,000.0 & 7711 \\ & \\ & \\ 1,000.0 & 6200 \\ 250.0 & 7900 \\ 3,000.0 & 6000 \\ 500.0 & 6600 \\ 1,000.0 & 5900\end{array}$

This is the 1955 Geneva conference reactor rebuilt with increased power.

The Thai research reactor, TRR-1, built by Curtiss-

Wright and started up in 1962 , originally operated at $1000 \mathrm{~kW}(\mathrm{t})$. In $6 / 75$ the TRR-1 was shut down for conversion to TRR-1/M1, a TRIGA-Mark III system adapted for pool installation. The TRR-1/M1, with a power level of $2000 \mathrm{~kW}(\mathrm{t}) / 2000 \mathrm{MW}$ pulsing was commissioned $11 / 77$.

This TRIGA reactor was operated at the 1958 International Conference in Geneva prior to shipment to the University of Lovanium in 6/59. It is the first reactor to be operated on the African continent.

Originally planned as TRIGA-Mk I, reactor was upgraded to TRIGA-Mk II. Original export license was not used.

250.0 


\section{University Research and Teaching}

\section{Reactor name. Owner}

Location

\section{OPERABLE}

Austra Vienna Polytechnic Insuture

Brazıl University of Minas Geraıs

Brazıl Unıversıty of São Paulo

Canada McMaster University

China, Republic of National Tsing-Hua University

Finland, Instutute of Technology

Germany Instıtute for Nuclear Medicine

Vienna
Belo Honzonte

$11 / 24 / 59$

XR-028

$08 / 03 / 59$

São Paulo

XR-002

$01 / 22157$

(1)

Ontano

Hsinchu

Helsinkı

Heidelberg

$08 / 27 / 57$

$\mathrm{XR} 020$

$06 / 05 / 58$

XR-040

$04 / 05 / 6$

XR 060
Germany, FRM Gerschıng Technical

University of Munich

Iran University of Tehran

Italy University of Palermo

Italy University of Pavia

Japan Kınkı Unıversıty

Japan Musashı College of Technology

Japan Rukkyo University

Korea University of Kyung Hee

Netherlands Delft Technical University

Switzerland University of Basel
Maınz

Munich

Tehran

Palermo

Pavia

Higashı Osaka

Kawasakı

Yokosuka

Seoul

Delft

Basel

Principal
nuclear
contractor.

Reactor type

\section{GA}

TRIGA-Mk II

GA

TRIGA-Mk I

B\&W

Pool

AMF

Pool

$02 / 14 / 66$

$\mathrm{GE}$

Pool

GA

TRIGA-Mk II

GA

TRIGA-Mk I

$\mathrm{XR}-050$

$04 / 11 / 64$

XR-076

$02 / 26 / 7$

XR-004

$03 / 15 / 57$

\begin{tabular}{|c|c|c|}
\hline $\begin{array}{l}\text { Power } \\
\text { design } \\
\text { net } \\
\text { MW(e) }\end{array}$ & $\begin{array}{l}\text { Power } \\
\text { MW(t) }\end{array}$ & $\begin{array}{c}\text { Author- } \\
\text { ized } \\
\text { power } \\
\text { kW(t) }\end{array}$ \\
\hline
\end{tabular}

$00 \quad 00 \quad 2500 \quad 6200$

$00 \quad 00 \quad 1000 \quad 6000$

$\begin{array}{llll}00 & 00 & 50000 & 5700\end{array}$

$\begin{array}{llll}00 & 00 & 50000 & 5900\end{array}$

$\begin{array}{llll}00 & 00 & 10000 & 6200\end{array}$

$\begin{array}{llll}00 & 00 & 2500 & 6200\end{array}$

$00-00-2500$

6600

TRIGA-Mk II

GA

TRIGA-Mk I

AMF

Pool

$\mathrm{XR} 029$

$08 / 05 / 59$

XR 025

$01 / 07 / 59$

XR-056

$03 / 12 / 65$

XR-041

$04 / 18 / 61$

XR 037

$07 / 08 / 60$

$\mathrm{XR} 038$

$07 / 08 / 60$

XR-105

$11 / 18 / 75$

XR 003

$02 / 01 / 57$

AMF

Pool

AGN

201-110

GA

TRIGA-Mk II

AR

UTR-10

GA

TRIGA-Mk II

GA

TRIGA-Mk II

AGN

201

AMF

Pool (MTR)

$\begin{array}{llll}00 & 00 & 1000 & 6500\end{array}$

6500

$\begin{array}{llll}00 & 00 & 2500 & 7300\end{array}$

$\begin{array}{llll}00 & 00 & 40000 & 5700\end{array}$

$\begin{array}{llll}00 & 00 & 50000 & 6700\end{array}$

$\begin{array}{llll}00 & 00 & 00 & 6000\end{array}$

$00 \quad 00 \quad 2500$

$00 \quad 00$

00

6500

6111

$00 \quad 00$

1000

6301

00

00

1000

6112

00

8200

6304

20000

AGN

211100

TIG A-Mk I reactor was installed in 1966 In 1977, the reactor was shut down, dismantled, and moved to another building After the move, 11 was started up again in 1978 This operation was referred to as "TRIGA I" and "TRIGA II "

Fuel suppler being sought

Neglugible power Shut down for renewal of operatung license

Shut down for renewal of operatung hcense

Neglıgible power

\section{Neghgıble power}

The Netherlands research reactor was onginally operated at the Amsterdam Intemational Exhubition in 6/57, major portions of the exhibition reactor system were used to fabricate the present reactor

This reactor was operated in the International Science Section of the Brussels Information Exhibition, 4/15/58 to 10/1/58, prior to transfer to the University of Basel Negligible power 
Facility (Regulatory agency)

\section{OPERABLE}

Advanced Test Reactor Critical Facilty (DOE)

Argonne National Laboratory, Idaho Division (DOE)

Los Alamos National Laboratory (DOE)

Los Alamos National Laboratory (DOE)

Los Alamos National Laboratory (DOE)

Los Alamos National Laboratory (DOE)

Los Alamos National Laboratory (DOE)

Los Alamos National Laboratory (DOE)

Los Alamos National Laboratory (DOE)

Los Alamos National Laboratory (DOE)

Los Alamos Natuonal Laboratory (DOE)

Oak Rıdge Cntical Expenments Facility (DOE)

ORNL Pool Crutucal Assembly BSF Pool (DOE)

Rensselaer Polytechnic Insutute, Troy, NY (NRC)

SNL Crucal Assembly

\section{MILITARY}

\section{Facility (Regulatory agency)}

\section{OPERABLE}

Knolls Atomic Power Laboratory (DOE)

Nuclear Safety Facility, Rocky Flats Plant (DOE)

Nuclear Safety Facilty, Rocky Flats Plant (DOE)

Nuclear Safety Facilty, Rocky Flats Plant (DOE)

Nuclear Safety Facilty, Rocky Flats Plant (DOE)

\section{Equipment}

\begin{tabular}{cc}
\hline & No. of \\
No. & control \\
of & panels/ \\
cells & room
\end{tabular}

Location

ATRC

ZPPR

Big Ten

Comet

Flattop

Godiva-IV

Honeycomb

Mars

Planet

SHEBA

SKUA

CEF

PCA

CX

INEL Site, ID

Los Alamos, NM

Los Alamos, NM

Los Alamos, NM

Los Alamos, NM

Los Alamos, NM

Los Alamos, NM

Los Alamos, NM

Los Alamos, NM

Los Alamos, NM

Oak Rudge, TN

Oak Rudge, TN

Troy, NY

Kurtland AFB East, NM
Initial

critlcality

Abbreviation (yr mo)

ATRC

ANL-IDAHO

LANL, Kiva II

LANL, Kiva II

LANL, Kiva II

LANL, Kiva III

LANL, Kiva I

LANL, Kiva I

LANL, Kiva II

LANL, KIva I

LANL, Kiva III

OR-CEF

ORNL-PCA

Rensselaer

Sandia
6400

6900

7200

5200

5700

6700

5600

7400

8000

7800

5000

5800

6600

8909

\section{Subject of experiment or study. Comment}

ATR physics, core-loading and core-design measurements Basic fast reactor studies and mock-up for LMFBR $\mathrm{U}(10)$-metal cylhnder in thick metal reflector Cntical-configuration safety and neutronic tests Sphencal metal cores in thick metal reflector Fast neutron irradiation, pulse capability Flexible split table assembly Vertical table assembly machine

Vertical table assembly

Solution high energy burst assembly

Fast neutron irradiation, pulse capabilty

Operable

Operable

Critucal experument assembly

Operatung
Initial

criticality

(yr mo)
Subject of experiment or study. Comment

\begin{tabular}{ll} 
FCPE & Schenectady, NY \\
Honzontal/ & Golden, CO \\
Splt Table & \\
Solution & Golden, CO \\
Base & \\
Vertical/ & Golden, CO \\
Split Table & \\
Water & Golden, CO \\
Reflector & \\
\hline
\end{tabular}

\begin{tabular}{cc} 
Equipment \\
\hline No. & $\begin{array}{c}\text { No. of } \\
\text { control }\end{array}$ \\
of & panels/ \\
cells & room
\end{tabular}

Abbreviation

$\begin{array}{lll}2 & 1 & \text { KAPL } \\ 0 & 0 & \text { RFP-NSF } \\ 1 & 1 & \text { RFP-NSF } \\ 0 & 0 & \text { RFP-NSF } \\ 0 & 0 & \text { RFP-NSF }\end{array}$

7000

6500

6500

6500

6500
Full core physics expenment

Critical-configuration safety tests

Critical-configuration safety tests

Cntical-configuration safety tests

Cntucal-configuration safety tests 
REACTORS AND FACILITIES

SHUTDOWN OR DISMANTLED 


\section{REACTORS AND FACILITIES SHUTDOWN OR DISMANTLED}

\section{POWER REACTORS}

\section{A. Central-Station Electric Power Plants}

\begin{tabular}{|c|c|c|c|c|c|c|c|}
\hline \multirow[b]{2}{*}{ Location } & \multirow[b]{2}{*}{$\begin{array}{l}\text { Principal } \\
\text { nuclear } \\
\text { contractor }\end{array}$} & \multicolumn{5}{|c|}{ Power } & \multirow[b]{2}{*}{ Comment } \\
\hline & & Type & $\begin{array}{l}\text { MD capacity } \\
\text { net MW(e) }\end{array}$ & $\begin{array}{l}\text { Licensed } \\
M W(t)\end{array}$ & $\begin{array}{l}\text { Initial } \\
\text { criticality } \\
\text { (yr mo) }\end{array}$ & $\begin{array}{l}\text { Shutdown } \\
\text { permanently } \\
\text { (yr mo) }\end{array}$ & \\
\hline Punta Higuera, PR & Comb & $\begin{array}{l}\text { Bollng water, } \\
\text { integral } \\
\text { nuclear } \\
\text { superheat }\end{array}$ & 165 & 500 & 6400 & 6800 & \\
\hline Рarr, SC & West & $\begin{array}{l}\text { Pressure tube, } \\
\text { heavy water }\end{array}$ & 170 & 640 & 6300 & 6701 & $\begin{array}{l}\text { The last CVTR shutdown occurred Jan 24, } 1967 \text { A } \\
\text { license amendment issued June } 14,1967 \text {, authonzes } \\
\text { CVNPA to possess but not operate the CVTR }\end{array}$ \\
\hline Morns, IL & GE & Bolhng water & 2000 & 7000 & 5900 & 7810 & \\
\hline Elk River, MN & $\mathrm{AC}$ & Bollng water & 220 & 582 & 6200 & 6802 & $\begin{array}{l}\text { The Elk River Reactor was shut down due to technical } \\
\text { problems in Febraary } 1968, \text { in } 1974 \text {, dismantling and } \\
\text { removal of this facilty was completed }\end{array}$ \\
\hline $\begin{array}{l}\text { Lagoona Beach, } \\
\text { MI }\end{array}$ & PRDC & $\begin{array}{l}\text { Sodum } \\
\text { cooled, } \\
\text { fast }\end{array}$ & 609 & 2000 & 6300 & 7209 & \\
\hline Platteville, CO & GA & $\begin{array}{l}\text { High tem } \\
\text { perature, } \\
\text { gas cooled }\end{array}$ & 3300 & 8420 & 7401 & 8908 & \\
\hline Hallam, NE & AI & $\begin{array}{l}\text { Sodum } \\
\text { graphite }\end{array}$ & 750 & 2400 & 6200 & 6409 & $\begin{array}{l}\text { The Hallam Nuclear Power Faculty was shut down in } \\
\text { September } 1964 \text { due to moderator-can falures } \\
\text { Entombment of the reactor was completed in } 1968\end{array}$ \\
\hline Eureka, CA & GE & Bolling water & 650 & 2420 & 6300 & 7607 & \\
\hline Buchanan, NY & $\mathrm{B} \& \mathrm{~W}$ & $\begin{array}{l}\text { Pressunzed } \\
\text { water }\end{array}$ & 2650 & 6150 & 6200 & 7410 & $\begin{array}{l}\text { In the Consolddated Edison Indian Point Station, the } \\
615 \mathrm{MW}(\mathrm{t}) \text { was increased by an oll-fired superheater } \\
\text { to produce } 265 \mathrm{MW}(\mathrm{e}) \text { net }\end{array}$ \\
\hline La Crosse, WI & $\mathrm{AC}$ & Bodling water & 480 & 1650 & 6707 & 8704 & \\
\hline Sroux Falls, SD & $\mathrm{AC}$ & Bolling water & 585 & 1900 & 6400 & 6709 & $\begin{array}{l}\text { The Pathfinder Plant has been shut down since } \\
\text { November } 1967 \text { On Sept } 9,1968, \text { Northern States } \\
\text { Power Company announced plans to mstall gas-fired } \\
\text { bolers for operation the summer of } 1969\end{array}$ \\
\hline Peach Botiom, PA & GA & $\begin{array}{l}\text { High tem } \\
\text { perature } \\
\text { gas cooled }\end{array}$ & 400 & 1150 & 6600 & 7410 & \\
\hline
\end{tabular}

Peach Bottom Atomic Power Station, Unit 1 (Philadelphia Electnc Co) [50-171] Station (AEC and Puerto Rico

Water Resources Authonty)

Carolinas-Virginia Tube Reactor (Carolinas-Virginia Nuclear

Power Associates, Inc )

Dresden Nuclear Power Station,

Unit 1 (Commonwealth Edison

Elk Ruver Reactor (AEC and Rural

Cooperatuve Power Association)

Ennco Fermi Atomic Power Plant

Unit 1 (Power Reactor

Development Co ) [50-16]

Fort St Vrain Nuclear Generating

Station (Public Service Company

of Colorado) [50-267]

Hallam Nuclear Power Faclity,

Sheldon Station (AEC and

Consumers Public Power District)

Humboldt Bay Power Plant,

Unit 3 (Pacific Gas \&

Electric Co ) [50-133]

Indian Point Station, Unit 1

(Consolidated Edison Co of

New York, Inc ) [50-3]

La Crosse (Genoa) Nuclear

Generating Station (Dairyland

Power Cooperative) [50-409]

Pathfinder Atomic Plant

(Northem States Power Co)

\section{PART 1 CIVILIAN REACTORS (DOMESTIC)}

an 24, $1967 \mathrm{~A}$ cense amendment issued June 14,1967, authonzes erate the CVIR in Febnuary 1968, in 1974, dismantling and eptember 1964 due to Entombment of the reactor was completed in 1968

the Consolidated Edison Indian Point Station, the $615 \mathrm{MW}(\mathrm{t})$ was increased by an oll-fired superheater to produce $265 \mathrm{MW}(\mathrm{e})$ net 1967 On Sept 9,1968, Northem States Power Company announced plans to install gas-fired gas cooled 


\section{POWER REACTOR}

PART 1 CIVILIAN REACTORS (DOMESTIC)

\section{A. Central-Station Electric Power Plants (Continued)}

\section{Name (licensee) [docket number]}

Piqua Nuclear Power Facility (AEC and City of Piqua)

Rancho Seco Nuclear Generatung Station, Unit 1 (Sacramento Municipal Utulty District) [50-312]

Shippingport Atomic Power Station (DOE and Duquesne Light Co )
Shoreham Nuclear Power Station (Long Island Lughung Co ) [50-322] Three Mule Island Nuclear Stawon,

Unit 2 (GPU Nuclear Corp) [50-320]

\section{B. Dual-Purpose Plants}

\section{Name (owner)}

N Reactor (DOE)

\section{Broothaven, NY}

Middletown, PA

$B \& W$
Principal nuclear contractor

AI

B\&W

West

est

Type

Organic

cooled and

moderated

Pressunzed

water

Pressunzed
water

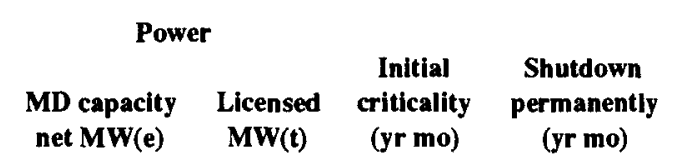

114

8730

27720

600

2360

5700
7409

yr mo)

6300

6600

8906

8210

8200

24360

8502

9107

Pressunzed

9060

27720

7800

7903

\section{Comment}

The dismantlement program for the Piqua Nuclear Power Facility was completed in February 1969

Plant shut down permanently $6 / 7 / 89$ because of public vote Fuel unloaded Licensee has applied for possession-only license

The first core for the Shippingport station began power operation in 1957, with a capacity ratung of $60 \mathrm{MW}$ (e) The second core began power operation in 1965, with a capacity rating of $90 \mathrm{MW}(\mathrm{e})$ The third core, a light water breeder reactor (LWBR), began power operation in 1977, with a capacity ratung of $60 \mathrm{MW}(\mathrm{e})$ Owned by the Department of Energy, the reactor plant was shut down on Oct 1 1982 Defueling was completed in September 1984 Decommissioning was completed in December 1989, and the site was released for use without any restrictions

Possession only license, 7/20/91

The Three Mule Island Nuclear Station, Unit 2, has been shut down since the $3 / 28 / 79$ accident Core removal is finished

\section{Location}

Principal

nuclear contractor

Hanford Site, WA
WHC
Power

$\begin{array}{ccc}\text { Capacity } & \text { Licensed } & \begin{array}{c}\text { Initial } \\ \text { criticality }\end{array} \\ \text { net MW(e) } & \text { MW(t) } & \text { (yr mo) }\end{array}$

Shut down (yr mo)

4000

\section{Comment}

9100
N Reactor, a DOE-owned reactor for production of special nuclear materials, also produced steam that was supplied to an adjacent electnc generatung plant, owned and operated by Washungton Public Power

Supply System The reactor has, with the decision to nun the K Reactor at Savannah River, been directed to transition from standby to the termination mode in preparation for decommissionin 


\section{Propulsion (Maritime)}

$\begin{array}{lccccccc}\text { Name and/or owner } & \text { Nuclear designer } & \text { Shipbuilder } & \text { Type } & \begin{array}{c}\text { Maximum } \\ \text { shaft } \\ \text { horsepower }\end{array} & \begin{array}{c}\text { Licensed } \\ \text { power } \\ \text { MW(e) }\end{array} & \begin{array}{c}\text { Start- } \\ \text { up } \\ \text { (yr mo) }\end{array} & \begin{array}{c}\text { Shut- } \\ \text { down } \\ \text { (yr mo) }\end{array} \\ \begin{array}{l}\text { Nuclear Shp SAVANNAH } \\ \text { (Mantime Adminstration) }\end{array} & \text { B\&W } & \text { NYSC } & \begin{array}{c}\text { Pressunzed } \\ \text { water }\end{array} & 22,000 & 80 & 6100 & 7100\end{array}$

\section{EXPERIMENTAL POWER-REACTOR SYSTEMS}

\section{A. Electric-Power Systems}

\begin{tabular}{|c|c|c|c|c|c|c|c|c|c|}
\hline $\begin{array}{l}\text { Name (Regulatory agency). } \\
\text { Location }\end{array}$ & $\begin{array}{c}\text { Power } \\
\text { capacity } \\
\text { net } k W(e)\end{array}$ & $\begin{array}{l}\text { Power } \\
\text { licensed } \\
\text { kW(t) }\end{array}$ & $\begin{array}{l}\text { Authorized } \\
\text { power } \\
\text { kW(t) }\end{array}$ & $\begin{array}{c}\text { Power } \\
\text { MD capacity } \\
\text { net } M W(e)\end{array}$ & $\begin{array}{l}\text { Power } \\
\text { licensed } \\
M W(t)\end{array}$ & $\begin{array}{l}\text { Initial } \\
\text { criticality } \\
\text { (yr mo) }\end{array}$ & $\begin{array}{l}\text { Shut- } \\
\text { down } \\
\text { (yr mo) }\end{array}$ & $\begin{array}{l}\text { Designation. } \\
\text { Type. } \\
\text { Principal } \\
\text { nucl. contr. }\end{array}$ & Comment \\
\hline $\begin{array}{l}\text { Bollung Reactor Experiment } \\
\text { No } 1 \text { (DOE) INEL Site, } \\
\text { ID }\end{array}$ & 00 & 1,4000 & 00 & 00 & 00 & 5300 & 5400 & $\begin{array}{l}\text { BORAX-1 } \\
\text { Bolling water } \\
\text { ANL }\end{array}$ & \\
\hline $\begin{array}{l}\text { Boulung Reactor Expenment } \\
\text { No } 5 \text { (DOE) INEL Site, } \\
\text { ID }\end{array}$ & 2,6000 & 20,0000 & 00 & 00 & 00 & 6200 & 6400 & $\begin{array}{l}\text { BORAX-5 } \\
\text { Bolling water, } \\
\text { integral nuclear } \\
\text { superheat ANL }\end{array}$ & \\
\hline $\begin{array}{l}\text { Bouling Reactor Expenments } \\
\text { (DOE) INEL Site, ID }\end{array}$ & 2,4000 & 15,5000 & 00 & 00 & 00 & 5400 & 5806 & $\begin{array}{l}\text { BORAX-2, 3,-4 } \\
\text { Bolling water } \\
\text { ANL }\end{array}$ & $\begin{array}{l}\text { This facility was onginally bult and operated in } \\
1954 \text { as the Bollng Reactor Expenment No } 2 \\
\text { (BORAX 2) With the addition of a turbogenerator, } \\
\text { it operated durng } 1955 \text { as BORAX-3 and on } \\
7 / 17 / 55 \text { produced sufficient electncity to light and } \\
\text { power Archo, ID-a U S first BORAX-4, a } \\
\text { further modification, operated from } 12 / 56 \text { to } 6 / 58 \text {, } \\
\text { when the expenment was shut down }\end{array}$ \\
\hline $\begin{array}{l}\text { ESADA Vallecitos Expen- } \\
\text { mental Superheat } \\
\text { Reactor (NRC) } \\
\text { Pleasanton, CA }\end{array}$ & 00 & 17,0000 & 00 & 00 & 00 & 6300 & 6702 & $\begin{array}{l}\text { EVESR } \\
\text { Lught-water } \\
\text { moderated, } \\
\text { superheater GE }\end{array}$ & $\begin{array}{l}\text { Owner Empire States Atomic Development } \\
\text { Associates and General Electric Company }\end{array}$ \\
\hline $\begin{array}{l}\text { Expenmental Beryllium } \\
\text { Oxide Reactor (DOE) } \\
\text { INEL Site, ID }\end{array}$ & 00 & 10,0000 & 00 & 00 & 00 & & & $\begin{array}{l}\text { EBOR Gas cooled, } \\
\text { BeO moderated } \\
\text { GA }\end{array}$ & $\begin{array}{l}\text { The EBOR reactor experiment was terminated in } \\
\text { December } 1966 \text { pnor to the completion of } \\
\text { construction }\end{array}$ \\
\hline $\begin{array}{l}\text { Expenmental Boulng } \\
\text { Water Reactor (DOE) } \\
\text { Argonne, IL }\end{array}$ & 4,0000 & 100,0000 & 00 & 00 & 00 & 5600 & 6706 & $\begin{array}{l}\text { EBWR Bolung } \\
\text { water ANL }\end{array}$ & $\begin{array}{l}\text { The EBWR achieved } 100,000 \mathrm{~kW}(\mathrm{t}) 11 / 11 / 62 \\
\text { Operation of EBWR in the Boiling Water Program } \\
\text { was closed out } 12 / 62 \text { The reactor was used in } \\
\text { support of the Plutonum Recycle Program and } \\
\text { attaned crutucalty using plutonum as its prncipal } \\
\text { fuel } 9 / 22 / 65 \text { In support of that program, it operated } \\
\text { at power levels as high as } 70,000 \mathrm{~kW}(\mathrm{t}) \text { Operation } \\
\text { in that program was completed } 6 / 67\end{array}$ \\
\hline
\end{tabular}




\section{EXPERIMENTAL POWER-REACTOR SYSTEMS}

PART 1 CIVILIAN REACTORS (DOMESTIC)

\section{A. Electric-Power Systems (Continued)}

\begin{tabular}{|c|c|c|c|c|c|c|c|c|}
\hline $\begin{array}{c}\text { Name (Regulatory agency). } \\
\text { Location }\end{array}$ & $\begin{array}{c}\text { Power } \\
\text { capacity } \\
\text { net } k W(e)\end{array}$ & $\begin{array}{c}\text { Power } \\
\text { licensed } \\
\mathbf{k W}(t)\end{array}$ & $\begin{array}{l}\text { Authorized } \\
\text { power } \\
\text { kW(t) }\end{array}$ & $\begin{array}{c}\text { Power } \\
\text { MD capacity } \\
\text { net MW(e) }\end{array}$ & $\begin{array}{c}\text { Power } \\
\text { licensed } \\
\text { MW(t) }\end{array}$ & $\begin{array}{l}\text { Initial } \\
\text { criticality } \\
\text { (yr mo) }\end{array}$ & $\begin{array}{l}\text { Shut- } \\
\text { down } \\
\text { (gr mo) }\end{array}$ & $\begin{array}{l}\text { Designation. } \\
\text { Type. } \\
\text { Principal } \\
\text { nucl. contr. }\end{array}$ \\
\hline $\begin{array}{l}\text { Experimental Breeder } \\
\text { Reactor No. I (DOE). } \\
\text { INEL Site, ID }\end{array}$ & 150.0 & $1,400.0$ & 0.0 & 0.0 & 0.0 & 5100 & 6400 & $\begin{array}{l}\text { EBR-1. NaK- } \\
\text { cooled, fast. } \\
\text { ANL }\end{array}$ \\
\hline
\end{tabular}

Experimental Gas

$21,900.0 \quad 84,300.0 \quad 0.0$

0.0

Oak Ridge, TN

Experimental Organic
Cooled Reactor (DOE).

INEL Site, ID

Heavy Water

Components Test

Reactor (DOE).

SRL, Aiken, SC

Homogeneous Reactor

Experiment No. 1

(DOE). Oak

Ridge, TN

Homogeneous Reactor

Experiment No. 2

(DOE). Oak

Ridge, TN

\section{Los Alamos Molten}

Plutonium Reactor

Experiment (DOE).

Los Alamos, NM

Los Alamos, NM

Pressurized heavy water. Du Pont

5400 HRE-1. Aqueous

homogeneous

solution $\left(\mathrm{UO}_{2} \mathrm{SO}_{4}\right)$

ORNL

6100 HRE-2. Aqueous

homogeneous

solution

$\left(\mathrm{UO}_{2} \mathrm{SO}_{4}\right)$.

ORNL

LAMPRE-1.

Fast molten

plutonium

fueled, sodium cooled. LANL

$\begin{array}{llll}0.0 & 2,000.0 & 0.0 & 0.0\end{array}$

\section{Comment}

In a trial nun 12/21/51 and 12/22/51, EBR-1 generated the world's first electric power from nuclear energy and was the first to demonstrate, in $7 / 53$, the feasibility of breeding and the compatibility with breeding economy of sodium-potassium alloy as a liquid-metal coolant. It operated with a plutoniumbearing core (Mark IV) from 11/62 to 12/63. The reactor was decommissioned and dismantled early in 1964. The facility was dedicated as a historic landmark $8 / 26 / 66$. It is open to the public from

Memorial Day weekend to Labor day weekend, annually.

The EGCR project was terminated 1/66 prior to the completion of construction.

\section{EOCR construction was terminated $12 / 62$. The}

facility was mothballed prior to operation. 


\begin{tabular}{|c|c|c|c|c|c|c|c|c|c|}
\hline $\begin{array}{l}\text { Los Alamos Power Reactor } \\
\text { Experiment No. } 2 \text { (DOE). } \\
\text { Los Alamos, NM }\end{array}$ & 0.0 & $1,000.0$ & 0.0 & 0.0 & 0.0 & 5900 & 5900 & $\begin{array}{l}\text { LAPRE-2. } \\
\text { Aqueous } \\
\text { homogeneous } \\
\text { (phosphoric } \\
\text { acid). LANL }\end{array}$ & \\
\hline $\begin{array}{l}\text { Molten Salt Reactor } \\
\text { Experiment (DOE). } \\
\text { Oak Ridge, TN }\end{array}$ & 0.0 & $8,000.0$ & 0.0 & 0.0 & 0.0 & 6500 & 6900 & $\begin{array}{l}\text { MSRE. Single } \\
\text { region, graphite } \\
\text { moderated. } \\
\text { ORNL }\end{array}$ & \\
\hline $\begin{array}{l}\text { Organic Moderated } \\
\text { Reactor Experiment } \\
\text { (DOE). INEL Site, ID }\end{array}$ & 0.0 & $12,000.0$ & 0.0 & 0.0 & 0.0 & 5700 & 6300 & $\begin{array}{l}\text { OMRE. Organic } \\
\text { cooled and } \\
\text { moderated. AI }\end{array}$ & $\begin{array}{l}\text { OMRE demonstrated the technical and economic } \\
\text { feasibility of using liquid hydrocarbon terphenyls } \\
\text { as coolant and/or moderator. }\end{array}$ \\
\hline $\begin{array}{l}\text { Plutonium Recycle } \\
\text { Test Reactor (DOE). } \\
\text { Richland, WA }\end{array}$ & 0.0 & $70,000.0$ & 0.0 & 0.0 & 0.0 & 6000 & 6900 & $\begin{array}{l}\text { PRTR. Pressure } \\
\text { tube, heavy } \\
\text { water mod- } \\
\text { erated and } \\
\text { cooled. WHC }\end{array}$ & \\
\hline $\begin{array}{l}\text { Saxton Nuclear } \\
\text { Experimental Reactor } \\
\text { Project (DOE). } \\
\text { Saxton, PA }\end{array}$ & $3,000.0$ & $23,500.0$ & 0.0 & 0.0 & 0.0 & 6200 & 7200 & $\begin{array}{l}\text { No Desg. } \\
\text { Pressurized } \\
\text { water. West. }\end{array}$ & Owner: Saxton Nuclear Experimental Corp. \\
\hline $\begin{array}{l}\text { Sodium Reactor } \\
\text { Experiment (DOE). } \\
\text { Santa Susana, CA }\end{array}$ & $5,700.0$ & $20,000.0$ & 0.0 & 0.0 & 0.0 & 5700 & 6402 & $\begin{array}{l}\text { SRE. Sodium } \\
\text { graphite. AI }\end{array}$ & $\begin{array}{l}\text { SRE operated at } 20 \mathrm{MW}(\mathrm{t}) \text { until shut down } 2 / 64 \text { for } \\
\text { modification to permit an increase in power level to } \\
30 \mathrm{MW}(\mathrm{t}) \text {. On } 12 / 2 / 66 \text {, deactivation of SRE was } \\
\text { announced. Owners: DOE and Southem Califormia } \\
\text { Edison Co. }\end{array}$ \\
\hline $\begin{array}{l}\text { Southwest Experimental } \\
\text { Fast Oxide Reactor } \\
\text { (NRC). Strickler, AR }\end{array}$ & 0.0 & $20,000.0$ & 0.0 & 0.0 & 0.0 & 6900 & 7200 & $\begin{array}{l}\text { SEFOR. Sodium } \\
\text { cooled, fast. } \\
\text { GE }\end{array}$ & Owner: Southwest Atomic Energy Associates. \\
\hline $\begin{array}{l}\text { Ultra High } \\
\text { Temperature Reactor } \\
\text { Experiment (DOE). } \\
\text { Los Alamos, NM }\end{array}$ & 0.0 & $3,000.0$ & 0.0 & 0.0 & 0.0 & 6800 & 7000 & $\begin{array}{l}\text { UHTREX. Helium } \\
\text { cooled. LANL }\end{array}$ & \\
\hline $\begin{array}{l}\text { Vallecitos Boiling } \\
\text { Water Reactor (NRC). } \\
\text { Pleasanton, CA }\end{array}$ & $5,000.0$ & $33,000.0$ & 0.0 & 0.0 & 0.0 & 5700 & 6312 & $\begin{array}{l}\text { VBWR. Boiling } \\
\text { water. GE }\end{array}$ & $\begin{array}{l}\text { Owner: General Electric Company and Pacific Gas \& } \\
\text { Electric Co. }\end{array}$ \\
\hline
\end{tabular}

\section{B. Space Nuclear Auxillary Power (SNAP)}

\begin{tabular}{|c|c|c|c|c|c|c|c|c|}
\hline $\begin{array}{l}\text { SNAP-02 Developmental } \\
\text { System (DOE). } \\
\text { Santa Susana, CA }\end{array}$ & 0.0 & 0.0 & 0.0 & 0.0 & 50.0 & 6100 & 6300 & $\begin{array}{l}\text { S2DS. NaK- } \\
\text { cooled. AI }\end{array}$ \\
\hline $\begin{array}{l}\text { SNAP-02 Experimental } \\
\text { Reactor (DOE). } \\
\text { Santa Susana, CA }\end{array}$ & 0.0 & 0.0 & 0.0 & 0.0 & 50.0 & 5900 & 6000 & $\begin{array}{l}\text { SER. NaK- } \\
\text { cooled. AI }\end{array}$ \\
\hline $\begin{array}{l}\text { SNAP-02/10A TSF } \\
\text { Shielding Experiment } \\
\text { (DOE). Oak Ridge, TN }\end{array}$ & 0.0 & 0.0 & 0.0 & 0.0 & 10.0 & 6700 & 7300 & $\begin{array}{l}\text { SNAP-TSF. } \\
\text { NaK-cooled. } \\
\text { AI-ORNL }\end{array}$ \\
\hline
\end{tabular}




\section{EXPERIMENTAL POWER-REACTOR SYSTEMS}

\section{B. Space Nuclear Auxlliary Power (SNAP) (Continued)}

Name (Regulatory agency).
Location

SNAP-08 Developmental

Reactor (DOE).

Santa Susana, CA

SNAP-08 Experiments

Reactor (DOE).

Santa Susana, CA

SNAP-10A Flight

System (DOE)

In orbit, USA

\section{SNAP-10A Flight}

System (DOE).

Oak Ridge, TN

SNAP-10A Flight

System Ground Test

No. 1 (DOE).

Santa Susana, CA

SNAP-10A Flight

System Ground Test

No. 3 (DOE).

Santa Susana, CA

\section{Space Propulsion}

Fuel Element Test

0.0

Bed (DOE).

NRDS, NV

Fuel Element Test

Reactor (DOE).

NRDS, NV

Fuel Element Test

Reactor (DOE).

NRDS, NV

Ground Experimental

Engine Experiment (DOE). NRDS, NV

Power

Power Authorized

capacity

licensed

Power
MD capacity
net $M W(e)$

$\mathbf{k W ( t )}$

0.0

$\begin{array}{ll}0.0 & 0.0\end{array}$

0.0

0.0

0.5

0.0

0.0

0.0

0.5

0.5

0.5

\section{PART 1 CIVILIAN REACTORS (DOMESTIC)}

Power

MW(t)

Initial

Shut-
down
(yr mo)

Designation.

600.0

6800

6900

Type.

Principal

600.0

6200

6500

S8DR. NaK

cooled. AI

$6500 \quad$ SIOFS-4. NaK-
39.0

6500

cooled. AI

39.0

(Spare)

39.0

6400

6400

S10FS-5

NaK-cooled.

AI

S10FS-1.

NaK-cooled.

AI

39.0

6400

6600

S10FS-3.

$\mathrm{NaK}-$ cooled.

AI

See comment for SNAP-10 Flight System, S1OFS-4.

10FS-4 operated in orbit during April-May 1965.

Operation terminated unexpectedly after 43 days at power, probably owing to a sequence of failures of electrical components of the spacecraft with

resulting spurious commands shutting down the reactor. An identical ground test unit, S10FS-3. operated successfully for more than a year before being shut down in 1966.

\begin{tabular}{cccc}
0.0 & 7200 & 7200 & $\begin{array}{c}\text { NF-1. Open cycle, } \\
\text { gaseous } \\
\text { hydrogen. } \\
\text { LANL } \\
\text { Pewee-1. Open } \\
\text { cycle, liquid } \\
\text { hydrogen. LANL } \\
\text { Pewee-2. Open } \\
\text { cycle, liquid } \\
\text { hydrogen. LANL } \\
\text { XE-Backup. } \\
\text { Open cycle, } \\
\text { liquid hydrogen. } \\
\text { AG-West. }\end{array}$ \\
\hline 0.0 & Indef. & 6800 & 7300
\end{tabular}




\begin{tabular}{|c|c|c|c|c|c|c|c|c|}
\hline $\begin{array}{l}\text { Ground Expenmental } \\
\text { Engme Expermment } \\
\text { (DOE). NRDS, NV }\end{array}$ & 00 & 00 & $1,100,0000$ & 00 & 00 & 6800 & 6900 & $\begin{array}{l}\text { XE-Prume Open } \\
\text { cycle, hquid } \\
\text { hydrogen, } \\
\text { AG-West. }\end{array}$ \\
\hline $\begin{array}{l}\text { Nuclear Rocket Engune } \\
\text { Reactor Expenment } \\
\text { (NERVA) (DOE) } \\
\text { NRDS, NV }\end{array}$ & 00 & 00 & 109,6000 & 00 & 00 & 6400 & 6400 & $\begin{array}{l}\text { NRX-A2 Open } \\
\text { cycle, hquid } \\
\text { hydrogen } \\
\text { AG-West }\end{array}$ \\
\hline $\begin{array}{l}\text { Nuclear Rocket Engune } \\
\text { Reactor Experiment } \\
\text { (NERVA) (DOE) } \\
\text { NRDS, NV }\end{array}$ & 00 & 00 & $1,100,0000$ & 00 & 00 & 6500 & 6500 & $\begin{array}{l}\text { NRX-A3 Open } \\
\text { cycle, liquid } \\
\text { hydrogen } \\
\text { AG-West }\end{array}$ \\
\hline $\begin{array}{l}\text { Nuclear Rocket Engune } \\
\text { Reactor Expenment } \\
\text { (NERVA) (DOE) } \\
\text { NRDS, NV }\end{array}$ & 00 & 00 & $1,120,0000$ & 00 & 00 & 6600 & 6600 & $\begin{array}{l}\text { NRX-A5 Open } \\
\text { cycle, liquid } \\
\text { hydrogen } \\
\text { AG-West }\end{array}$ \\
\hline $\begin{array}{l}\text { Nuclear Rocket Engine } \\
\text { Reactor Experiment } \\
\text { (NERVA) (DOE) } \\
\text { NRDS, NV }\end{array}$ & 00 & 00 & $1,199,0000$ & 00 & 00 & 6700 & 6700 & $\begin{array}{l}\text { NRX A6 Open } \\
\text { cycle, hquid } \\
\text { hydrogen } \\
\text { AG-West }\end{array}$ \\
\hline $\begin{array}{l}\text { Nuclear Rocket Reactor } \\
\text { Engine System Test } \\
\text { (NERVA) (DOE) } \\
\text { NRDS, NV }\end{array}$ & 00 & 00 & $1,155,0000$ & 00 & 00 & 6600 & 6600 & $\begin{array}{l}\text { NRX A4/EST } \\
\text { Open cycle, } \\
\text { liquid hydrogen } \\
\text { AG-West }\end{array}$ \\
\hline $\begin{array}{l}\text { Nuclear Rocket Reactor } \\
\text { Expenment (DOE) } \\
\text { NRDS, NV }\end{array}$ & 00 & 00 & 70,0000 & 00 & 00 & 5900 & 5900 & $\begin{array}{l}\text { Kıw1 A Open } \\
\text { cycle, gaseous } \\
\text { hydrogen LANL }\end{array}$ \\
\hline $\begin{array}{l}\text { Nuclear Rocket Reactor } \\
\text { Expenment (DOE) } \\
\text { NRDS, NV }\end{array}$ & 00 & 00 & 85,0000 & 00 & 00 & 6000 & 6000 & $\begin{array}{l}\text { Kiw1 A Pnme } \\
\text { Open cycle, } \\
\text { gaseous hydrogen } \\
\text { LANL }\end{array}$ \\
\hline $\begin{array}{l}\text { Nuclear Rocket Reactor } \\
\text { Expenment (DOE) } \\
\text { NRDS, NV }\end{array}$ & 00 & 00 & 100,0000 & 00 & 00 & 6000 & 6000 & $\begin{array}{l}\text { Kiw1-A3 Open } \\
\text { cycle, gaseous } \\
\text { hydrogen LANL }\end{array}$ \\
\hline $\begin{array}{l}\text { Nuclear Rocket Reactor } \\
\text { Expenment (DOE) } \\
\text { NRDS, NV }\end{array}$ & 00 & 00 & 300,0000 & 00 & 00 & 6100 & 6100 & $\begin{array}{l}\text { Kiw B 1A Open } \\
\text { cycle, gaseous } \\
\text { hydrogen LANL }\end{array}$ \\
\hline $\begin{array}{l}\text { Nuclear Rocket Reactor } \\
\text { Expenment (DOE) } \\
\text { NRDS, NV }\end{array}$ & 00 & 00 & 90,0000 & 00 & 00 & 6200 & 6200 & $\begin{array}{l}\text { Kiw口 B IB Open } \\
\text { cycle, lquid } \\
\text { hydrogen LANL }\end{array}$ \\
\hline $\begin{array}{l}\text { Nuclear Rocket Reactor } \\
\text { Expenment (DOE) } \\
\text { NRDS, NV }\end{array}$ & 00 & 00 & 500,0000 & 00 & 00 & 6200 & 6200 & $\begin{array}{l}\text { Kiw1 B4A Open } \\
\text { cycle, hquid } \\
\text { hydrogen LANL }\end{array}$ \\
\hline $\begin{array}{l}\text { Nuclear Rocket Reactor } \\
\text { Expenment (DOE) } \\
\text { NRDS, NV }\end{array}$ & 00 & 00 & $1,000,0000$ & 00 & 00 & 6400 & 6400 & $\begin{array}{l}\text { Kiwı B4D Open } \\
\text { cycle, hquid } \\
\text { hydrogen LANL }\end{array}$ \\
\hline $\begin{array}{l}\text { Nuclear Rocket Reactor } \\
\text { Expenment (DOE) } \\
\text { NRDS, NV }\end{array}$ & 00 & 00 & $1,000,0000$ & 00 & 00 & 6400 & 6400 & $\begin{array}{l}\text { Kiwı B4D Open } \\
\text { cycle, hquid } \\
\text { hydrogen LANL }\end{array}$ \\
\hline
\end{tabular}




\section{Space Propulsion (Continued)}

\begin{tabular}{|c|c|c|c|c|c|c|c|c|}
\hline $\begin{array}{c}\text { Name (Regulatory agency). } \\
\text { Location }\end{array}$ & $\begin{array}{c}\text { Power } \\
\text { capacity } \\
\text { net } k W(e)\end{array}$ & $\begin{array}{c}\text { Power } \\
\text { llcensed } \\
\text { kW(t) }\end{array}$ & $\begin{array}{l}\text { Authorized } \\
\text { power } \\
k W(t)\end{array}$ & $\begin{array}{c}\text { Power } \\
\text { MD capacity } \\
\text { net } M W(e)\end{array}$ & $\begin{array}{c}\text { Power } \\
\text { Ilcensed } \\
\text { MW(t) }\end{array}$ & $\begin{array}{c}\text { Initial } \\
\text { criticality } \\
\text { (yr mo) }\end{array}$ & $\begin{array}{l}\text { Shut- } \\
\text { down } \\
\text { (yr mo) }\end{array}$ & $\begin{array}{c}\text { Type. } \\
\text { Principal } \\
\text { nucl. contr. }\end{array}$ \\
\hline $\begin{array}{l}\text { Nuclear Rocket Reactor } \\
\text { Expenment (DOE) } \\
\text { NRDS, NV }\end{array}$ & 00 & 00 & 950,0000 & 00 & 00 & 6400 & 6400 & $\begin{array}{l}\text { Kiw1-B4E Open } \\
\text { cycle, lquid } \\
\text { hydrogen LANL }\end{array}$ \\
\hline $\begin{array}{l}\text { Nuclear Rocket Reactor } \\
\text { Expenment (DOE) } \\
\text { NRDS, NV }\end{array}$ & 00 & 00 & $1,070,0000$ & 00 & 00 & 6500 & 6500 & $\begin{array}{l}\text { Phoebus 1A Open } \\
\text { cycle, lquid } \\
\text { hydrogen LANL }\end{array}$ \\
\hline $\begin{array}{l}\text { Nuclear Rocket Reactor } \\
\text { Experiment (DOE) } \\
\text { NRDS, NV }\end{array}$ & 00 & 00 & $1,400,0000$ & 00 & 00 & 6700 & 6700 & $\begin{array}{l}\text { Phoebus 1B Open } \\
\text { cycle, liquid } \\
\text { hydrogen LANL }\end{array}$ \\
\hline $\begin{array}{l}\text { Nuclear Rocket Reactor } \\
\text { Expenment (DOE) } \\
\text { NRDS, NV }\end{array}$ & $0 a$ & 00 & $4,200,0000$ & 00 & 00 & 6800 & 6800 & $\begin{array}{l}\text { Phoebus } 2 \mathrm{~A} \text { Open } \\
\text { cycle, liquid } \\
\text { hydrogen LANL }\end{array}$ \\
\hline
\end{tabular}

\section{TEST, RESEARCH, AND UNIVERSITY REACTORS}

\section{A. General Irradiation Test}

\begin{tabular}{|c|c|c|c|c|c|c|c|c|}
\hline $\begin{array}{l}\text { Engineenng Test Reactor } \\
\text { (DOE) INEL, ID }\end{array}$ & 00 & 00 & 175,0000 & 00 & 00 & 5700 & 8100 & $\begin{array}{l}\text { ETR Tank } \\
\text { KE-GE }\end{array}$ \\
\hline $\begin{array}{l}\text { General Electric Testung } \\
\text { Reactor (NRC) } \\
\text { Pleasanton, CA }\end{array}$ & 00 & 00 & 50,0000 & 00 & 00 & 5800 & 7700 & $\begin{array}{l}\text { GETR Tank } \\
\text { Owner }\end{array}$ \\
\hline $\begin{array}{l}\text { Matenals Testung Reactor } \\
\text { (DOE) INEL, ID }\end{array}$ & 00 & 00 & 40,0000 & 00 & 00 & 5200 & 7000 & $\begin{array}{l}\text { MTR Tank } \\
\text { ORNL-ANL- } \\
\text { Blaw-Knox }\end{array}$ \\
\hline $\begin{array}{l}\text { Plum Brook Reactor Facility } \\
\text { (NRC) Sandusky, OH }\end{array}$ & 00 & 00 & 60,0000 & 00 & 00 & 6100 & 7400 & $\begin{array}{l}\text { NASA-TR } \\
\text { Tank NASA }\end{array}$ \\
\hline $\begin{array}{l}\text { Westunghouse Testung Reac- } \\
\text { tor (DOE) Waltz Mill, PA }\end{array}$ & 00 & 00 & 60,0000 & 00 & 00 & 5900 & 6200 & $\begin{array}{l}\text { WTR Tank } \\
\text { Owner }\end{array}$ \\
\hline
\end{tabular}

Reactor was shut down in 1973 for modifications and insertion of Sodium Loop Safety Facility (SLSF) loop Operatuon resumed in 1975 Deactivated in 1981 and no longer operable

In August 1958 the MTR was operated with an experimental plutonium core at power levels up to $30,000 \mathrm{~kW}(\mathrm{t})$ It demonstrated the abılity of plutonium fuel elements to perform satusfactonly in a high-flux research or test reactor Operation as a test reactor was terminated $6 / 30 / 69$, and a

Plutonium-240 (Phoenix) core was run in FY 1970 Reactor was decommıssioned in 1974 


\title{
B. High-Power Research and Test
}

\begin{tabular}{|c|c|c|c|c|c|c|c|c|c|}
\hline $\begin{array}{l}\text { Ames Laboratory Research } \\
\text { Reactor (DOE). Ames, IA }\end{array}$ & 0.0 & 0.0 & $5,000.0$ & 0.0 & 0.0 & 6500 & 7700 & $\begin{array}{r}\text { ALRR. Heavy } \\
\text { water. AMF }\end{array}$ & \\
\hline $\begin{array}{l}\text { Argonne Research Reactor } \\
\text { (DOE). Argonne, IL }\end{array}$ & 0.0 & 0.0 & $5,000.0$ & 0.0 & 0.0 & 5400 & 7900 & $\begin{array}{l}\text { CP-5. Heavy } \\
\text { water. ANL }\end{array}$ & \\
\hline $\begin{array}{l}\text { Babcock \& Wilcox } \\
\text { Nuclear Development } \\
\text { Center Test Reactor } \\
\text { (NRC). Lynchburg, VA }\end{array}$ & 0.0 & 0.0 & $6,000.0$ & 0.0 & 0.0 & 6400 & 7100 & $\begin{array}{l}\text { BAWTR. } \\
\text { Pool. Owner }\end{array}$ & \\
\hline $\begin{array}{l}\text { Cintichem, Inc. Reactor } \\
\text { (NRC). Sterling Forest, } \\
\text { NY }\end{array}$ & 0.0 & 0.0 & $5,000.0$ & 0.0 & 0.0 & 6100 & 9000 & CINR. Pool. AMF. & Decommissioning plan has been submitted to NRC. \\
\hline $\begin{array}{l}\text { Industrial Reactor Labora- } \\
\text { tories, Inc. (NRC). } \\
\text { Plainsboro, NJ }\end{array}$ & 0.0 & 0.0 & $5,000.0$ & 0.0 & 0.0 & 5800 & 7500 & IRL. Pool. AMF & \\
\hline $\begin{array}{l}\text { Oak Ridge Research } \\
\text { Reactor (DOE). } \\
\text { Oak Ridge, TN }\end{array}$ & 0.0 & 0.0 & $30,000.0$ & 0.0 & 0.0 & 5800 & 8700 & $\begin{array}{l}\text { ORR. Tank. } \\
\text { ORNL }\end{array}$ & $\begin{array}{l}\text { The servo system for the ORR, completed in August } \\
\text { 1986, consisted of a programmable controller and } \\
\text { associated auxiliary equipment for signal } \\
\text { conditioning and input/output functions. The } \\
\text { functions of the programmable controller were: } \\
\text { (1) demand control; (2) primary and pool flow and } \\
\text { differential temperature calculations; (3) reactor } \\
\text { heat power calculations; (4) neutron flux } \\
\text { calibration; and (5) demand-flux comparison and } \\
\text { rod positioning. A new ORR safety system was } \\
\text { installed in 1986. Three channels of neutron flux } \\
\text { information were arranged in two-out-of-three } \\
\text { coincidences to permit online testing and repair and } \\
\text { to prevent an unnecessary shutdown, should a } \\
\text { single channel have failed. }\end{array}$ \\
\hline $\begin{array}{l}\text { Sandia Engineering } \\
\text { Reactor (DOE). Kirland } \\
\text { AFB, NM }\end{array}$ & 0.0 & 0.0 & $5,000.0$ & 0.0 & 0.0 & 6100 & 7000 & $\begin{array}{l}\text { SER. Tank. } \\
\text { Sandia }\end{array}$ & \\
\hline
\end{tabular}

\section{Safety Research and Test}

\author{
King Intense Neutron \\ Generator (DOE). \\ Los Alamos, NM \\ Kiwi-Transient Test \\ Reactor (DOE)
}

0.0

0.0

0.0

0.0

0.0

7200
$7700 \quad$ Kinglet.
Homogeneous.

0.0

0.0

0.0

6500
6500 LANL
Kiwi-TTR.
Kiwi/
NERVA.
LANL

servo system for the ORR, completed in August functions of the programmable controller were:

(1) demand control; (2) primary and pool flow and demperature calculations; (3) reactor information were arranged in two-Out-of three coincidences to permit online testing and repair and to prevent an unnecessary shutdown, should a 


\section{Safety Research and Test (Continued)}

\begin{tabular}{|c|c|c|c|c|c|c|c|c|}
\hline $\begin{array}{c}\text { Name (Regulatory agency). } \\
\text { Location }\end{array}$ & $\begin{array}{c}\text { Power } \\
\text { capacity } \\
\text { net } k W(e)\end{array}$ & $\begin{array}{l}\text { Power } \\
\text { licensed } \\
\mathbf{k W}(t)\end{array}$ & $\begin{array}{l}\text { Authorized } \\
\text { power } \\
\text { kW(t) }\end{array}$ & $\begin{array}{c}\text { Power } \\
\text { MD capacity } \\
\text { net } M W(e)\end{array}$ & $\begin{array}{c}\text { Power } \\
\text { licensed } \\
\text { MW(t) }\end{array}$ & $\begin{array}{c}\text { Initial } \\
\text { criticality } \\
\text { (yr mo) }\end{array}$ & $\begin{array}{l}\text { Shut. } \\
\text { down } \\
\text { (yr mo) }\end{array}$ & $\begin{array}{c}\text { Designation. } \\
\text { Type. } \\
\text { Principal } \\
\text { nucl. contr. }\end{array}$ \\
\hline $\begin{array}{l}\text { Loss of Fluid Test (DOE). } \\
\text { INEL, ID }\end{array}$ & 0.0 & 0.0 & $55,000.0$ & 0.0 & 0.0 & 7800 & 8507 & $\begin{array}{l}\text { LOFT. } \\
\text { Pressurized } \\
\text { water. } \\
\text { EG\&G-ID }\end{array}$ \\
\hline $\begin{array}{l}\text { SNAP-10A Transient } \\
\text { Test No. } 2 \text { (DOE). } \\
\text { NEL, ID }\end{array}$ & 0.0 & 0.0 & 0.0 & 0.0 & 0.0 & 6500 & 6601 & $\begin{array}{l}\text { SNAPTRAN-2 } \\
\text { Be-reflected } \\
\text { SNAP-10A. } \\
\text { AI-PPC }\end{array}$ \\
\hline
\end{tabular}

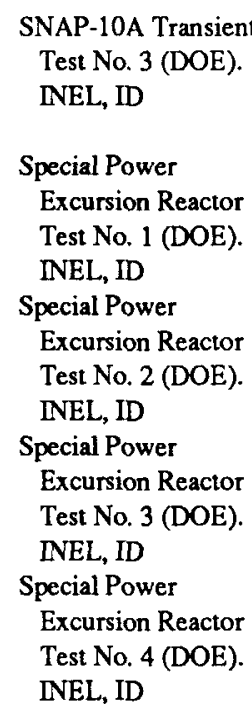

\footnotetext{
Test No. 4 (DOE).
}

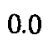

0.0

5500

6000

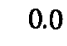

0.0

5800

6200
6400

SNAPTRAN-3 $\mathrm{H}_{2} \mathrm{O}$-reflected SNAP-10A. PPC-AI SPERT-1. Open tank. PPC

\section{$6500 \quad$ SPERT -2.} Pressurized water. PPC

6800 SPERT-3. Pressurized water. PPC

$7000 \quad$ SPERT-4. Pool.
INC

\section{Comment}

LOFT covered most of the concerns related to lightwater safety. The first tests were done for NRC, and the last eight were done for a consortium of OECD countries and the U.S. The last two fissionproduct-release tests measured release and transport of fission products. There was an intentional core damage causing a partial meltdown. Facility has been inactivated and placed in cold standby.

The SNAPTRAN series of experiments was designed to develop, in a land-based environment, safety information on space auxiliary power reactors

through excursion testing at various temperatures and rates of reactivity insertion. The destructive experiments approach the maximum credible accidents postulated for SNAP reactor systems. SNAPTRAN-1 was converted to SNAPTRAN-2 for destructive testing $1 / 66$. 


\section{General Research}

\begin{tabular}{|c|c|c|c|c|c|c|c|c|c|}
\hline $\begin{array}{l}\text { Accelerator Pulsed Fast } \\
\text { Crucal Assembly (DOE) } \\
\text { La Jolla, CA }\end{array}$ & 00 & 00 & 10 & 00 & 00 & 6700 & 7300 & $\begin{array}{l}\text { APFA-III } \\
\text { Fast GA }\end{array}$ & $\begin{array}{l}\text { The APFA-III was previously operated as the } \\
\text { KUKLA Prompt Cnucal Assembly at Lawrence } \\
\text { Luvermore National Laboratory at Livermore, CA }\end{array}$ \\
\hline $\begin{array}{l}\text { Amencan Standard Inc } \\
\text { (NRC) Mountain } \\
\text { View, CA }\end{array}$ & 00 & 00 & 00 & 00 & 00 & 5800 & 6000 & $\begin{array}{l}\text { UTR-1 } \\
\text { Graphute/water } \\
\text { AS, Inc }\end{array}$ & $\begin{array}{l}\text { This reactor was shipped abroad for exhibition } \\
\text { purposes in the USAEC Atoms for Peace Exhibit } \\
\text { in the Tokyo International Trade Far in 1959, and } \\
\text { in Cairo, Egypt, and Lahore, Pakistan, in } 1960\end{array}$ \\
\hline $\begin{array}{l}\text { Annular Core Pulsed } \\
\text { Reactor (DOE) } \\
\text { Kunland AFB, } \\
\text { East, NM }\end{array}$ & 00 & 00 & 00 & 00 & 00 & 6700 & 7700 & $\begin{array}{l}\text { ACPR U-Zr } \\
\text { hydnde } G A\end{array}$ & $\begin{array}{l}\text { In } 1977 \text { the Annular Core Pulsed Reactor (ACPR) } \\
\text { was shut down After replacement of fuel and } \\
\text { other modffications, the unit was renamed the } \\
\text { Annular Core Research Reactor }\end{array}$ \\
\hline $\begin{array}{l}\text { Argonne CP-3, rebult as } \\
\text { CP-3' (DOE) } \\
\text { Palos Park, IL }\end{array}$ & 00 & 00 & 3000 & 00 & 00 & 4400 & 6300 & $\begin{array}{l}\text { CP-3' Heavy } \\
\text { water Met Lab }\end{array}$ & \\
\hline $\begin{array}{l}\text { Argonne Low Power } \\
\text { Research Reactor (DOE) } \\
\text { Argonne, IL }\end{array}$ & 00 & 00 & 2500 & 00 & 00 & 6200 & 7000 & $\begin{array}{l}\text { Juggemaut } \\
\text { Graphte/water } \\
\text { ANL }\end{array}$ & $\begin{array}{l}\text { After the assembly and operation of this reactor in } \\
\text { the govemment exhbbit at Geneva in } 9 / 58 \text {, it was } \\
\text { dismantled and retumed to ANL, where it was } \\
\text { rebult as a } 250-\mathrm{kW}(\mathrm{t}) \text { Juggemaut }\end{array}$ \\
\hline $\begin{array}{l}\text { Argonne Natuonal } \\
\text { Laboratory (DOE) } \\
\text { Argonne, IL }\end{array}$ & 00 & 00 & 00 & 00 & 00 & 5700 & 7200 & $\begin{array}{l}\text { AGN 201-108 } \\
\text { Homog } \\
\text { soldd AGN }\end{array}$ & \\
\hline $\begin{array}{l}\text { Argonne Nuclear } \\
\text { Assembly for University } \\
\text { Training (DOE) } \\
\text { Argonne, IL }\end{array}$ & 00 & 00 & 100 & 00 & 00 & 5700 & 7200 & $\begin{array}{l}\text { Argonaut (CP 11) } \\
\text { Graphite/water } \\
\text { ANL }\end{array}$ & \\
\hline $\begin{array}{l}\text { Argonne Thermal } \\
\text { Source Reactor (DOE) } \\
\text { Argonne, IL }\end{array}$ & 00 & 00 & 100 & 00 & 00 & 5700 & 8800 & $\begin{array}{l}\text { ATSR Thermal } \\
\text { ANL }\end{array}$ & \\
\hline $\begin{array}{l}\text { Atomics Intemauonal } \\
\text { (NRC) Canoga Park, IL }\end{array}$ & 00 & 00 & 00 & 00 & 00 & 5700 & 5800 & $\begin{array}{l}\text { L-47 } \\
\text { Homogeneous } \\
\text { AI }\end{array}$ & \\
\hline $\begin{array}{l}\text { Babcock \& Wulcox } \\
\text { Lynchburg Pool } \\
\text { Reactor (NRC) } \\
\text { Lynchburg, VA }\end{array}$ & 00 & 00 & 1,0000 & 00 & 00 & 5800 & 8100 & $\begin{array}{l}\text { LRP Pool } \\
\text { Owner }\end{array}$ & \\
\hline $\begin{array}{l}\text { Battelle Memonal } \\
\text { Instutute (NRC) } \\
\text { West Jefferson, OH }\end{array}$ & 00 & 00 & 2,0000 & 00 & 00 & 5600 & 7400 & $\begin{array}{l}\text { BRR Pool } \\
\text { AMF }\end{array}$ & \\
\hline $\begin{array}{l}\text { Brookhaven Graphite } \\
\text { Research Reactor (DOE) } \\
\text { Upton, NY }\end{array}$ & 00 & 00 & 20,0000 & 00 & 00 & 5000 & 6900 & $\begin{array}{l}\text { BGRR } \\
\text { Air cooled, } \\
\text { graphite } \\
\text { moderated } \\
\text { AU, Inc }\end{array}$ & \\
\hline $\begin{array}{l}\text { Brookhaven Neutron Source } \\
\text { Reactor No } 1 \text { (DOE) } \\
\text { Upton, NY }\end{array}$ & 00 & 00 & 1000 & 00 & 00 & 5800 & 7000 & $\begin{array}{l}\text { SCHIZO Tank } \\
\text { AU, Inc }\end{array}$ & \\
\hline
\end{tabular}




\section{TEST, RESEARCH, AND UNIVERSITY REACTORS}

PART 1 CIVILIAN REACTORS (DOMESTIC)

\section{General Research (Continued)}

\begin{tabular}{|c|c|c|c|c|c|c|c|c|c|}
\hline $\begin{array}{c}\text { Name (Regulatory agency). } \\
\text { Location }\end{array}$ & $\begin{array}{c}\text { Power } \\
\text { capacity } \\
\text { net } k W(e)\end{array}$ & $\begin{array}{l}\text { Power } \\
\text { licensed } \\
k W(t)\end{array}$ & $\begin{array}{l}\text { Authorized } \\
\text { power } \\
\text { kW(t) }\end{array}$ & $\begin{array}{c}\text { Power } \\
\text { MD capacity } \\
\text { net } M W(e)\end{array}$ & $\begin{array}{l}\text { Power } \\
\text { licensed } \\
M W(t)\end{array}$ & $\begin{array}{c}\text { Initial } \\
\text { criticality } \\
\text { (yr mo) }\end{array}$ & $\begin{array}{l}\text { Shut- } \\
\text { down } \\
\text { (yr mo) }\end{array}$ & $\begin{array}{l}\text { Designation. } \\
\text { Type. } \\
\text { Principal } \\
\text { nucl. contr. }\end{array}$ & Comment \\
\hline $\begin{array}{l}\text { Brookhaven Neutron Source } \\
\text { Reactor No } 2 \text { (DOE) } \\
\text { Upton, NY }\end{array}$ & 00 & 00 & 1000 & 00 & 00 & 6500 & 7000 & $\begin{array}{l}\text { PHRENIC Tank } \\
\text { AU, Inc }\end{array}$ & \\
\hline $\begin{array}{l}\text { Bulk Shueldıng Reactor } \\
\text { (DOE) Oak Rudge, TN }\end{array}$ & 00 & 00 & 2,0000 & 00 & 00 & 5000 & 9100 & $\begin{array}{l}\text { BSR Pool } \\
\text { ORNL }\end{array}$ & \\
\hline $\begin{array}{l}\text { Chicago Pile } 1 \text {, rebult as } \\
\text { CP } 2 \text { (DOE) } \\
\text { Chicago, IL }\end{array}$ & 00 & 00 & 02 & 00 & 00 & 4200 & 5400 & $\begin{array}{l}\text { CP-2 Graphite } \\
\text { Met Lab }\end{array}$ & $\begin{array}{l}\text { In } 1943 \text { the Manhattan Engineer District } \\
\text { disassembled Chicago Pile } 1 \text { and rebuilt it at Palos } \\
\text { Park, IL, as Chicago Ple } 2 \text { CP-2 had a thermal- } \\
\text { power level of } 10 \mathrm{~kW}\end{array}$ \\
\hline $\begin{array}{l}\text { Curuss-Wright Nuclear } \\
\text { Research Laboratory of } \\
\text { the Commonwealth of } \\
\text { Pennsylvanıa (NRC) } \\
\text { Quehanna, PA }\end{array}$ & 00 & 00 & 1,0000 & 00 & 00 & 5800 & 6600 & $\begin{array}{l}\text { CWRR Pool } \\
\text { Owner }\end{array}$ & \\
\hline $\begin{array}{l}\text { DOE Demonstration } \\
\text { Reactor (DOE) } \\
\text { Oak Rudge, TN }\end{array}$ & 00 & 00 & 100 & 00 & 00 & 6900 & 6900 & $\begin{array}{l}\text { Demo Reac } \\
\text { Pool Lockheed }\end{array}$ & $\begin{array}{l}\text { This reactor was formerly called the Latun Amencan } \\
\text { Demonstration Reactor and was operated initually } \\
\text { in São Paulo, Brazul, 10/69 It is currently in } \\
\text { storage at Oak Rudge }\end{array}$ \\
\hline $\begin{array}{l}\text { European-Asıan } \\
\text { Exhubit Program (DOE) } \\
\text { Oak Rudge, TN }\end{array}$ & 00 & 00 & 100 & 00 & 00 & 6300 & 6900 & $\begin{array}{l}\text { EAEP Pool } \\
\text { Lockheed }\end{array}$ & $\begin{array}{l}\text { This reactor was operated in the USAEC Atoms for } \\
\text { Peace Exhibit in Vienna, Austna, 6/63, Belgrade, } \\
\text { Yugoslavia, 9/63, Madnd, Spaun, 4/64, Lusbon, } \\
\text { Porugal, 4/65, Utrecht, Netherlands, 3/66, Dublin, } \\
\text { Ireland, 9/66 to 10/66, Ankara, Turkey, 4/67 to } \\
\text { 5/67, Tehran, Iran, 11/67 to 12/67, Taipe1, Tawwan, } \\
\text { 4/68 to 5/68, Seoul, Korea, 9/68 to 10/68, Manula, } \\
\text { Phulppines, 2/69 to 3/69, and Bucharest, Romania, } \\
\text { 10/69 }\end{array}$ \\
\hline $\begin{array}{l}\text { Fast Neutron Source } \\
\text { Reactor (DOE) } \\
\text { Upton, NY }\end{array}$ & 00 & 00 & 00 & 00 & 00 & 6700 & 7000 & $\begin{array}{l}\text { BNL/FS-1 } \\
\text { Fast BNL }\end{array}$ & \\
\hline $\begin{array}{l}\text { General Atomics } \\
\text { Technologies (DOE) } \\
\text { San Diego, CA }\end{array}$ & 00 & 00 & 500 & 00 & 00 & 6000 & 6000 & $\begin{array}{l}\text { TRIGA-Mk I } \\
\text { U-Zr hydnde } \\
\text { Owner }\end{array}$ & $\begin{array}{l}\text { This TRIGA-Mk II was operated at the New Delhı } \\
\text { World Agncultural Faur in } 1960 \text { It was dismantled } \\
\text { for storage in Calffoma by Chevron USA } \\
\text { Corporatoon Owner World Agncultural Faur-U S } \\
\text { Exhubit Reactor }\end{array}$ \\
\hline $\begin{array}{l}\text { Health Physics Research } \\
\text { Reactor (DOE) Oak } \\
\text { Ridge. TN }\end{array}$ & 00 & 00 & 100 & 00 & 00 & 6200 & 9100 & $\begin{array}{l}\text { HPRR Fast } \\
\text { Burst. ORNL. }\end{array}$ & \\
\hline
\end{tabular}




\section{High Tempenature \\ Launce Test \\ Reactor (DOE)}

Richland WA

Illinos Instutute of Tech-

nology Research Insutute

(DOE) Chicago, IL

Kinetuc Expeniment on

Water Bollers (NRC)

Santa Susana, CA

\section{Luvermore Pool Type \\ Reactor (DOE)}

Luvermore, $C A$

uvermore Water Boller

(DOE) Livermore, CA

Lockheed Aureraft Corp

(NRC) Dawsonville, GA

Los Alamos Fast

Reactor (DOE)

Los Alamos, NM

Los Alamos LOPO

Reactor (DOE)

Los Alamos, NM

Los Alamos Water Boller (DOE)

Los Alamos, NM

Los Alamos Water

Boller (DOE)

Los Alamos, NM

Lourstans State

University Nuclear

Saence Center (DOE)

Baton Rouge, LA

Low Intensity Test

Reactor (DOE)

Oak Rudge, TN

Low Temperature Neutron

Irradiation Facilty (DOE)

Oak Ridge, TN

NASA Mock-Up Reactor

(NRC) Sandusky, OH

Neutron Radiography

Facility (DOE)

Hanford Sire, WA
6700

$7100 \quad$ HTLTR

Graphite

PNI

0

750

00

$6700 \quad$ ARR (L-54)

Homogeneous

00

00

00

00

$00 \quad 5600$

6700 KEWB

Homogeneous

AI

00

00

3,0000

00

00

5700

8000 LPTR Tank FW

00

00

05

00

00

5300

6100 LIWB

Homogeneous

$$
\text { AI }
$$

250

00

00

4600

5300

Pool Lockheed

plutine Fast, mercury cooled LANL

00

4400

4400

SUPO

Homogeneous

LANL

6600 SNARE Pool
Sandia
$00 \quad 5600$

Owner Armour Research Foundation

The KEWB reactor was operated by AI from 1956 to 1967 as the Kineuc Experiment on Water Boilers Owner Rockwell International No power listed transient

In 1965 and 1966 this reactor was operated at Sandra NM, as SNARE Pnor to that tume it was operated at DNEL as the Shield Test Pool Reactor (SUSIE) in the Aircraft Nuclear Propulsion Program from 1959 to 1962 It was shut down in 1966 and transferred to Louisiana State University 6/66, where it was never assembled Owner Phillips Petroleum Co ORNL

$9100 \quad$ LTNIF Pool ORNL

2500

7700 Lockheed NRF U-Zr

The Neutron Radiography Faculity was used to hydnde WHC examine post irradiation of reactor fuel columns and pin components The facility was shut down in 1990 and transferred to the surplus factities program for decontamination and decommissioning 


\section{General Research (Continued)}

Name (Regulatory agency).
Location
Northrop Corporate
Laboratones (NRC)
Hawthome, CA

Nuclear Effects Reactor

(DOE) NTS, NV

Nuclear Effects

Reactor (DOE)

San Drego, CA

Nuclear Examunation

Reactor (NRC)

Santa Susana, CA

Oak Rudge GraphiteReactor

(DOE) Oak Rudge, TN

Pawling Research Reactor

(NRC) Pawling, NY

Physical Constants Test

Reactor (DOE)

Ruchland, VA

Radiation Effects

Reactor (NRC)

Dawsonville, GA

\section{Rockwell}

Intemational (NRC)

Canoga Park, CA

Sandia Pulsed Reactor

(DOE) Kurland AFB

East, NM

$\begin{array}{ccc}\text { Power } & \text { Power } & \text { Authorized } \\ \text { capacity } & \text { licensed } & \text { power } \\ \text { net } k W(e) & k W(t) & k W(t)\end{array}$

00

00

00

00

00

00

00

00

00

00

30

00

00

00

5900

00

5200

Prompt burst

LLNL

$8000 \quad$ L-85 (AE-6)

Homogeneous

AI

00

00

3,5000

00

00

00

00

3,0000

00

00

5800

4300

6300

ORG

Graphne CL

PRR LWR

UNC

7200 PCTR Graphite

PNL

7000

RER Poo Lockheed
00

5800

00

6100
7400

L-77

Homogeneous

AI

6700 SPR Prompt

\section{Comment}

Owner Space Radiation Laboratory This TRIGA reactor was capable of being pulsed and of steadystate operation

Until mid-1967 FRAN was operated by LLNL at the Nevada Test Site, and untll 1970 it was operated in the former ML-1 reactor area at INEL In mid-1970 It was transferred back to LLNL Fuel was removed for processing

Ownership of this reactor was transferred to Nonh American Rockwell $12 / 1$ and was redesignated the Nuclear Examination Reactor or L-85 rather than AE-6 The AE-6, also designated WBNS, was built and first operated at Downey, CA It was moved to Santa Susana in 1956

Owner United Nuclear Corp

The RER was previously used in the terminated Aircraft Nuclear Propulsion Program A license authonzing Lockheed to operate the reactor as a commercial facilty was $15 s u e d 7 / 62$, and $8 / 62$ the USAF transferred the facllty to the General Services Administration Lockheed acquired title to the facility $3 / 65$ 


Shield Test and Irradiation
Reactor (DOE).
Santa Susana, CA
Thermal Test Reactor No. 2
(DOE).Richland, WA
Torrey Pines, TRIGA-
Mk III Reactor (NRC).
La Jolla, CA
Tower Shielding Reactor
(DOE). Oak Ridge, TN
UTR Test Reactor
(NRC). Mountain
View, CA
Westinghouse Nuclear
Train-ing Center (NRC).
Zion, IL

$\begin{array}{rrrr}0.0 & 0.0 & 1,000.0 & 0.0 \\ 0.0 & 0.0 & 0.1 & 0.0 \\ 0.0 & 0.0 & 1,500.0 & 0.0 \\ 0.0 & 0.0 & 500.0 & 0.0 \\ 0.0 & 0.0 & 0.0 & 0.0 \\ 0.0 & & & \\ & 0.0 & 10.0 & 0.0\end{array}$

$\begin{array}{ll}0.0 & 6100 \\ 0.0 & 5500 \\ 0.0 & 6600 \\ 0.0 & 5400 \\ 0.0 & 6100 \\ 0.0 & 7200\end{array}$

$7200 \quad$ STIR.
Pool. AI

$7200 \quad$ TTR-2. Graphite.

7300 PNL

7300 TRIGA-Mk III. Owner

5800 TSR. BSR-type in

tank. ORNL

6300 No Desg.

Graphite/water.

Owner

$8700 \quad$ WNTR. Tank.
West.

\section{E. Unlversity Research and Teaching}

Brigham Young

University (NRC).

Provo, UT

California Polytechnic

State University

(NRC). San Luis

Obispo, CA

$\begin{array}{cccccccc}0.0 & 0.0 & 0.0 & 0.0 & 0.0 & 6700 & 8700 & \begin{array}{c}\text { L-77. } \\ \text { Homogeneous. } \\ \text { AI }\end{array} \\ 0.0 & 0.0 & 0.0 & 0.0 & 0.0 & 7300 & 8000 \quad \begin{array}{c}\text { AGN-201-100. } \\ \text { Homog. } \\ \text { solid. AGN }\end{array}\end{array}$

California, Berkeley,

University of (NRC).

Berkeley, CA

California, Los

Angeles, University

of, School of Engi-

neering and Applied

Science (NRC).

Los Angeles, CA

Califomia, Santa

Barbara, University

of (NRC). Santa

Barbara, CA

Catholic University

of America (NRC).

Washington, D.C.

Colorado State

University (NRC).

1000.0

0.0

0.0

6600

8712 TRIGA-Mk III. $\mathrm{U}-\mathrm{Zr}$ hydride.

100.0

0.0

0.0

6000

$8400 \quad$ GA

Graphite/water. AMF
This reactor was previously designated STF for SNAP Shield Test Facility.

Owner: General Atomic.

Owner: American Radiator \& Standard Sanitary Corp.

Califormia Polytechnic State University received permit in $12 / 71$ to relocate AGN-201-100 and operate it on the university's campus. The unit was previously operated starting in 1956 at the Naval Postgraduate School, Monterey, CA. In 1980, AGN-201-100 was shut down and

decommissioned. 


\section{E. University Research and Teaching (Continued)}

Name (Regulatory agency).
Location

Columbia

University (NRC).

New York, NY

Delaware, University

of (NRC).

Newark, DE

Georgia Institute of

Technology (NRC)

Atlanta, GA

Kansas, University

of (NRC).

Lawrence, KS

Leland Stanford

University (NRC).

Palo Alto, CA

Memphis State

University (NRC).

Memphis, TN

Michigan State University

(NRC). East Lansing, MI

Power
capacity
net kW(e)

Power
licensed
kW(t)

Authorized
power
kW(t)

0.0

0.0

250.0

0.0

0.0

0.0

0.0

0.0

0.0

Power
MD capacity
net MW(e)

Power

licensed

MW(t)

Initial

criticality

(yr mo)

0.0

0.0

Licensed

8500

0.0

0.0

0.0

5800

7800

0.0

6800

8500

0.0

0.0

10.0

0.0

0.0

10.0

0.0

0.0

0.0

0.0

0.0

7700

6900

250.0

0.0

0.0

6100

8700

5900

7400

8500

8900

00

0.0

0.0

0.0

Mississippi State

University (NRC).
State College, MS

0.0

0.0

0.0

Nevada, University

of (NRC). Reno, NV

North Carolina State

University (NRC).

Raleigh, NC

L-77. Homogeneous.
RRR.

Homogeneous.

Owner-NSA

Designation.

Type.

Principal

TRIGA-Mk II.

GA

AGN-201-113.

Homog. solid.

AGN

AGN-201-104.

Homog. solid.

AGN

Model 4180.

Pool. BAC

No Desg.
Pool. GE

AGN-201-108.

Homog. solid.

AGN

U-Zr hydrid

The core of the Michigan State University reactor aperated in the University of Mlinois TRIGA

facility from 1960 until transferred in 1968. The reactor has been decommissioned. The license was terminated 4/5/90.

This reactor was originally operated by North Carolina State University as the Raleigh Research Reactor (RRR). It was transferred 3/66 to Mississippi State University for reactivation. The RRR was dismantlec by N.C. State in 1963. Owing to funding problems, this reactor was never activated. Late in 1981 it was shipped to Barnwell, SC, for disposal.
The Columbia University TRIGA-Mk II was licensed to operate by NRC. However, the City of New

Columbia University has not procured fuel. The Columbia University has not procin
license was terminated in 1985.

AGN-201-104 operated at the University of Akron (Ohio) from 1957 until transferred to the Georg that facility began in 1968. Decommissioning of AGN-201-104 was achieved in 1986 


\begin{tabular}{|c|c|c|c|c|c|c|c|c|c|}
\hline $\begin{array}{l}\text { Oklahoma, University } \\
\text { of (NRC) } \\
\text { Norman, OK }\end{array}$ & 00 & 00 & 00 & 00 & 00 & 5800 & 8800 & $\begin{array}{l}\text { AGN-211-102 } \\
\text { Homog solid, } \\
\text { pool AGN }\end{array}$ & Possession only license, 1988 \\
\hline $\begin{array}{l}\text { Oregon State } \\
\text { University (NRC) } \\
\text { Corvallis, OR }\end{array}$ & 00 & 00 & 00 & 00 & 00 & 5800 & 7400 & $\begin{array}{l}\text { AGN-201-114 } \\
\text { Homog solid } \\
\text { AGN }\end{array}$ & \\
\hline $\begin{array}{l}\text { Polytechnic } \\
\text { Instutute of New } \\
\text { York (NRC) } \\
\text { New York, NY }\end{array}$ & 00 & 00 & 00 & 00 & 00 & 6700 & 7400 & $\begin{array}{l}\text { AGN-201M-105 } \\
\text { Homog solid } \\
\text { AGN }\end{array}$ & $\begin{array}{l}\text { In } 1957-1962, \text { AGN-201M-105 was owned and } \\
\text { operated by the National Naval Medical Center, } \\
\text { Bethesda, MD Tile to the reactor was transferred } \\
\text { to New York University early in } 1964 \text { A license to } \\
\text { operate was issued 4/67 }\end{array}$ \\
\hline $\begin{array}{l}\text { Puerto Rico Nuclear } \\
\text { Center (DOE) } \\
\text { Mayaguez, PR }\end{array}$ & 00 & 00 & 00 & 00 & 00 & 5900 & 7900 & $\begin{array}{l}\text { L-77 } \\
\text { Homogeneous } \\
\text { AI }\end{array}$ & $\begin{array}{l}\text { Owner The Center for Environmental and Energy } \\
\text { Research (formerly Puerto Rico Nuclear Center) }\end{array}$ \\
\hline $\begin{array}{l}\text { Puerto Rico Nuclear } \\
\text { Center (DOE) } \\
\text { Mayaguez, PR }\end{array}$ & 00 & 00 & 20000 & 00 & 00 & 7200 & 7600 & $\begin{array}{l}\text { TRIGA-FLIP } \\
\text { Pool-TRIGA } \\
\text { core GA }\end{array}$ & $\begin{array}{l}\text { This reactor was operated at the Pueno Rico Nuclear } \\
\text { Center from } 1960 \text { to } 10 / 76, \text { it was converted to the } \\
\text { TRIGA-FLIP in } 1972 \text { It has been moved to the } \\
\text { Neutron Radiography Faculty at the National } \\
\text { Engineenng Laboratory in Idaho }\end{array}$ \\
\hline $\begin{array}{l}\text { Texas at Austun, University } \\
\text { of (NRC) Austın, TX }\end{array}$ & 00 & 00 & 2500 & 00 & 00 & 6300 & 9100 & $\begin{array}{l}\text { TRIGA-Mk I } \\
\text { U Zr hydnde } \\
\text { GA }\end{array}$ & Possession only license \\
\hline $\begin{array}{l}\text { Tuskegee Instutute (NRC) } \\
\text { Tuskegee, AL }\end{array}$ & 00 & 00 & 00 & 00 & 00 & 7400 & 8412 & $\begin{array}{l}\text { AGN-201 } 102 \\
\text { Homog } \\
\text { solıd AGN }\end{array}$ & $\begin{array}{l}\text { AGN 201-102 was operated at Oklahoma State } \\
\text { University, Stullwater, OK, from } 1957 \text { untl } \\
\text { transferred to Tuskegee Institute in 1972, there it } \\
\text { was licensed to operate but was never started up } \\
\text { Tuskegee Institute retumed the fuel to the } \\
\text { Department of Energy, and the operating license } \\
\text { was terminated 10/12/84 }\end{array}$ \\
\hline $\begin{array}{l}\text { Utah, University of (NRC) } \\
\text { Salt Lake City, UT }\end{array}$ & 00 & 00 & 00 & 00 & 00 & 5700 & 9100 & $\begin{array}{l}\text { AGN } 201107 \\
\text { U Zr hydnde } \\
\text { AGN }\end{array}$ & Being decommissioned \\
\hline $\begin{array}{l}\text { Virginia, University of } \\
\text { (NRC) Chariottesville, } \\
\text { VA }\end{array}$ & 00 & 00 & 00 & 00 & 00 & 7400 & 9100 & $\begin{array}{l}\text { CAVALIER } \\
\text { Type not } \\
\text { specified } \\
\text { Owner }\end{array}$ & Being decommissioned \\
\hline $\begin{array}{l}\text { Virginia Polytechnic } \\
\text { Insutute (NRC) } \\
\text { Blacksburg, VA }\end{array}$ & 00 & 00 & 1000 & 00 & 00 & 5900 & 8400 & $\begin{array}{l}\text { UTR-10 } \\
\text { Graphite/water } \\
\text { AS Inc }\end{array}$ & \\
\hline $\begin{array}{l}\text { Washington, University of } \\
\text { (NRC) Seattle, WA }\end{array}$ & 00 & 00 & 1000 & 00 & 00 & 6100 & 9000 & $\begin{array}{l}\text { Educator } \\
\text { Graphtte/water } \\
\text { AMF }\end{array}$ & Decommissioning plan has been submitted to NRC \\
\hline $\begin{array}{l}\text { West Virgunia } \\
\text { University (NRC) } \\
\text { Morgantown, WV }\end{array}$ & 00 & 00 & 00 & 00 & 00 & 5900 & 7200 & $\begin{array}{l}\text { AGN-211-103 } \\
\text { Homog sold, } \\
\text { pool AGN }\end{array}$ & \\
\hline $\begin{array}{l}\text { William Marsh Rice } \\
\text { University (NRC) } \\
\text { Houston, TX }\end{array}$ & 00 & 00 & 00 & 00 & 00 & 5900 & 6500 & $\begin{array}{l}\text { AGN-211-101 } \\
\text { Homog soldd, } \\
\text { pool AGN }\end{array}$ & \\
\hline $\begin{array}{l}\text { Wyoming, University of } \\
\text { (NRC) Laramie, WY }\end{array}$ & 00 & 00 & 00 & 00 & 00 & 5900 & 7400 & $\begin{array}{l}\text { L-77 } \\
\text { Homogeneous } \\
\text { AI }\end{array}$ & \\
\hline
\end{tabular}




\begin{tabular}{|c|c|c|c|c|c|c|c|}
\hline Name (all owned by DOE) & Designation & Location & $\begin{array}{l}\text { Nuclear } \\
\text { designer }\end{array}$ & Type & $\begin{array}{c}\text { Authorized } \\
\text { power } \\
\text { kW(t) }\end{array}$ & $\begin{array}{c}\text { Initial } \\
\text { criticality } \\
\text { (yr mo) }\end{array}$ & $\begin{array}{l}\text { Shut- } \\
\text { down } \\
\text { (yr mo) }\end{array}$ \\
\hline B Reactor & B Reactor & Richland, WA & Du Pont & Graphite & 0.0 & 4400 & 6800 \\
\hline C Reactor & C Reactor & Richland, WA & GE & Graphite & 0.0 & 5200 & 6900 \\
\hline D Reactor & D Reactor & Richland, WA & Du Pont & Graphite & 0.0 & 4400 & 6700 \\
\hline DR Reactor & DR Reactor & Richland, WA & GE & Graphite & 0.0 & 5000 & 6400 \\
\hline F Reactor & F Reactor & Richland, WA & Du Pont & Graphite & 0.0 & 4500 & 6500 \\
\hline H Reactor & H Reactor & Richland, WA & GE & Graphite & 0.0 & 4900 & 6500 \\
\hline KE Reactor & KE Reactor & Richland, WA & GE & Graphite & 0.0 & 5500 & 7100 \\
\hline KW Reactor & KW Reactor & Richland, WA & GE & Graphite & 0.0 & 5500 & 7000 \\
\hline R Reactor & R Reactor & $\begin{array}{l}\text { Savannah River Plant, } \\
\text { Aiken, SC }\end{array}$ & Du Pont & Heavy water & 0.0 & 5300 & 6400 \\
\hline
\end{tabular}

\section{PROCESS DEVELOPMENT}

\begin{tabular}{|c|c|c|c|c|c|c|c|c|}
\hline Hanford 305 Test Reactor & HTR & Richland, WA & Du Pont & Graphite & 0.0 & 4400 & 7600 & Negligible power. \\
\hline Lattice Test Reactor & LTR & $\begin{array}{l}\text { Savannah River Plant, } \\
\text { Aiken, SC }\end{array}$ & Du Pont & Heavy water & 1.0 & 6700 & 7900 & \\
\hline Process Development Pile & PDP & $\begin{array}{l}\text { Savannah River Plant, } \\
\text { Aiken, SC }\end{array}$ & Du Pont & Heavy water & 1.0 & 5300 & 7900 & \\
\hline SR 305-M Test Pile & Test Pile & $\begin{array}{l}\text { Savannah River Plant, } \\
\text { Aiken, SC }\end{array}$ & Du Pont & Graphite & 1.0 & 5300 & 8300 & $\begin{array}{l}\text { SR 305-M Test Pile was used to measure the } \\
\text { reactivity effects of components (fuel tubes, target } \\
\text { tubes, control rods, etc.) prior to use in Savannah } \\
\text { River (SR) reactors. In addition, the Test Pile was } \\
\text { used to measure the neutron absorption of mis- } \\
\text { cellaneous materials used at SR. The Test Pile has } \\
\text { been dismantled. }\end{array}$ \\
\hline $\begin{array}{l}\text { Standard Pile/Subcritical } \\
\text { Experimental Complex }\end{array}$ & SP/SE & $\begin{array}{l}\text { Savannah River } \\
\quad \text { Laboratory, Aiken, SC }\end{array}$ & Du Pont & Graphite & 2.0 & 5300 & 7900 & $\begin{array}{l}\text { The SP-an enriched uranium-fueled, graphite- } \\
\text { moderated, water-cooled reactor-supplied neutrons } \\
\text { for reactor-component-reactivity testing in the SE, } \\
\text { which was a graphite chamber on top of the SP. } \\
\text { Authorized power ranged from } 2 \text { to } 10 \mathrm{~kW}(\mathrm{t}) \text {. }\end{array}$ \\
\hline
\end{tabular}




\section{A. Remote Installations}

Reactors in the Army Power Program are designated to reflect mobility charactenstics, power range, development sequence, and field sequence. The first capital letter indicates mobility charactenstics $S$ (stationary operation), not designed for subsequent relocation; $P$ (portable), semimobile, stationary operation, capable of being dismantled and reassembled for use in successive locations, and $M$ (mobile) capable of being moved intact, or virtually intact, for use in successive locations The second capital letter indicates the power range as measured by design capacity for contınuous operation $\mathrm{L}$ (low),
100 to $1000 \mathrm{~kW}(\mathrm{e}), \mathrm{M}$ (medium), 1000 to $10,000 \mathrm{~kW}(\mathrm{e})$, and $\mathrm{H}$ (high), $10,000 \mathrm{~kW}(\mathrm{e})$ or more. Arabic numerals indicate order in which plants having the same moblity and power charactenstucs are inituated. If not followed by an additional letter, the designation indicates a prototype or pilot plant The last capital letter (when present) indicates the alphabetical order in which freld plants of a specific type are mitrated
Portable Medium Power Plant, No $3 \mathrm{~A}$

Stationary Meduum Power Plant No 1

Stationary Medium Power Plant No $1 \mathrm{~A}$
PM-3A

SM-1

SM-1A

MH-1A
Principal

\section{nuclear}

contractor.

Reactor type

Location

Sundance, WY

Martun

Pressunzed water

Camp Century,

Greenland

Alco

Pressunzed water
Power

capacity

net

$\mathbf{k W ( e )}$

1,0000

1,5600
Authorized

power
kW(t)

Initial

$k W(t)$

(yr mo)

9,3700

6200

10,0000

6000

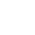

Shut-

down

(yr mo)

6800

6300

McMurdo Sound,

Antarctica

Fort Belvorr, VA Alco

Fort Greely, AK Alco
Martın

Pressunzed water

Pressunzed water

Pressunzed water

1,8550

1,6500

9,5100

6200

7300

10,0000

5700

6200

7300

20,2000

45,0000

Martun
Pressunzed water

10,0000

Power Plant
The PM 2A was shut down 7/9/63 and dismantled durng $4 / 64$ to $6 / 64$ The reactor vessel was then used by LNEL for NDT (nul ductility transiuon temperature) invesugauons of matenals that had been subjected to long-term imadiation Defects were sequentually introduced into the vessel wall dunng a senes of tests involving pressure and temperature conditions which exceeded the range permitted in operatung nuclear power plants The final test on 11/18/66 resulted in a bntule fracture under condiuons even more severe than those which had been previously predicted to cause fallure The test program con firmed laboratory data on the adequacy of reactor-operatıng limitations to prevent brittle fracture of a pressure vessel

The Army made the determination to shut down the SM 1A because the plant's demonstration and R\&D missions had been successfully completed and because of the ready avallabilty of cheaper conventional power at the site

The MH-1A was installed in the STURGIS (formerly the Liberty Ship CHARLES H CUGLE) at Mobile, AL Acceptance testing was performed at Fort Belvoir, VA, $4 / 67$ to $6 / 27 / 67$, when the Amy accepted the plant from the contractor In late $7 / 68$ the plant was deployed to Gatun Lake, Panama Canal Zone, and began producIng power to the Panama Canal power gnd on 10/5/68 The MH-1A is no longer avallable for service 


\section{B. Propulsion (Naval)}

SSN 1s Submarine (Nuclear Propulsion)

Name (all owned by U.S. Navy) Descnption

\section{NAUTILUS}

SEAWOLF PWR

SEAWOLF Sodium Reactor

SSN571

SKATE

SWORDFISH

SARGO

SEADRAGON

SKIPJACK*

TRITON (2 reaciors)

HALIBUT

SCAMP*

SCORPION

SCULPIN

SHARK*

SNOOK*

THRESHER

PERMIT

$\mathbb{N}$

SSN575
SSN575

SSN578

SSN579

SSN583

SSN584

SSN585

SSN586

SSN587

SSN588

SSN589

SSN590

SSN591

$\mathrm{SSN} 592$

SSN593

SSN594

SSN595

BARB*

TULLIBEE

GEORGE WASHINGTON*

PATRICK HENRY*

THEODORE ROOSEVELT*

ROBERT E LEE*

ABRAHAM LINCOLN*

POLLACK*

HADDO

JACK

DACE*

ETHAN ALLEN*

SAM HOUSTON

THOMAS A EDISON*

LAFAYETTE

THOMAS JEFFERSON*

ANDREW JACKSON

JOHN ADAMS*

JAMES MONROE*

NATHAN HALE*

HENRY CLAY

NATHANAEL GREENE

QUEENFISH

SEA DEVIL

GLENARD P LIPSCOMB*

SSN596

SSN597

SSN598

SSN599

SSBN600

SSN601

SSBN602

SSN603

SSN604

SSN605

SSN607

SSN609

SSN610

SSBN616

SSN618

SSBN619

SSBN620

SSBN622

SSBN623

SSBN625

SSBN636

SSN651

SSN664

SSN685

SSBN 15 Fleet Ballisuc Missule Submanne (Nuclear Propulsion)

\begin{tabular}{|c|c|c|c|}
\hline hipbuilder & $\begin{array}{l}\text { Start- } \\
\text { up }\end{array}$ & $\begin{array}{l}\text { Shut- } \\
\text { down }\end{array}$ & Comments \\
\hline Electnc boat (Groton) & 5400 & 8000 & \\
\hline Electric boat (Groton) & 6000 & 8600 & \\
\hline Electnc boat (Groton) & 5700 & 5900 & $\begin{array}{l}\text { The SEAWOLF, onginally commissioned with a sodium-cooled reactor in } \\
3 / 57 \text {, was recommissioned with a pressurized-water reactor on } 9 / 30 / 60\end{array}$ \\
\hline Electric Boat (Groton) & 5700 & 8500 & \\
\hline Portsmouth & 5800 & 8700 & \\
\hline Mare Island & 5800 & 8600 & \\
\hline Portsmouth & 5900 & 8300 & \\
\hline Electric Boat (Groton) & 5800 & 8900 & \\
\hline Electnc Boat (Groton) & 5900 & 6800 & \\
\hline Mare Island & 5900 & 7600 & \\
\hline Mare Island & 6100 & 8700 & \\
\hline Electnc Boat (Groton) & 6000 & 6800 & The SCORPION was lost in the Atlanuc 5/21/68 \\
\hline Ingalls & 6100 & 8900 & \\
\hline Newport News & 6000 & 8900 & \\
\hline Ingalls & 6100 & 8600 & \\
\hline Portsmouth & 6100 & 6300 & The THRESHER was lost in the Atlantic $4 / 10 / 63$ \\
\hline Mare Island & 6200 & 9000 & \\
\hline Mare Island & 6200 & 8900 & \\
\hline Ingalls & 6300 & 8900 & \\
\hline Electrnc Boat (Groton) & 6000 & 8700 & \\
\hline Electnc Boat (Groton) & 5900 & 8400 & \\
\hline Electnc Boat (Groton) & 6000 & 8300 & \\
\hline Mare Island & 6000 & 8100 & \\
\hline Newpon News & 6000 & 8300 & \\
\hline Electnc Boat (Groton) & 6000 & 8100 & \\
\hline NYSC & 6300 & 8800 & \\
\hline NYSC & 6400 & 9000 & \\
\hline Portsmouth & 6500 & 8900 & \\
\hline Ingalls & 6300 & 8800 & \\
\hline Electric Boat (Groton) & 6100 & 8200 & \\
\hline Newport News & 6100 & 9100 & \\
\hline Electnc Boat (Groton) & 6100 & 8300 & \\
\hline Electnc Boat (Groton) & 6300 & 9100 & \\
\hline Newpon News & 6200 & 8400 & \\
\hline Mare Island & 6300 & 8800 & \\
\hline Portsmouth & 6400 & 8800 & \\
\hline Newpon News & 6300 & 9000 & \\
\hline Electric Boat (Groton) & 6300 & 8600 & \\
\hline Newport News & 6300 & 9000 & \\
\hline Portsmouth & 6400 & 8600 & \\
\hline Newport News & 6600 & 9000 & \\
\hline Newport News & 6800 & 9100 & \\
\hline Electnc Boat (Groton) & 7400 & 9000 & \\
\hline
\end{tabular}




\section{A. Electric-Power Experiments and Prototypes}

\begin{tabular}{|c|c|c|c|c|c|c|c|c|}
\hline Name (Owner) & Designation & Location & $\begin{array}{c}\text { Principal } \\
\text { nuclear } \\
\text { contractor. } \\
\text { Reactor type }\end{array}$ & $\begin{array}{c}\text { Power } \\
\text { capacity } \\
\text { net } \\
\mathbf{k W}(\mathbf{e})\end{array}$ & $\begin{array}{c}\text { Authorized } \\
\text { power } \\
\text { kW(t) }\end{array}$ & $\begin{array}{c}\text { Initial } \\
\text { criticality } \\
\text { (yr mo) }\end{array}$ & $\begin{array}{c}\text { Shutdown } \\
\text { (yr mo) }\end{array}$ & Comment \\
\hline $\begin{array}{l}\text { Gas Cooled Reactor Expenment } \\
\text { (DOE) }\end{array}$ & GCRE & INEL Site, ID & $\begin{array}{l}\text { AGN } \\
\text { Gas cooled, light } \\
\text { water moderated }\end{array}$ & 00 & 2,2000 & 6000 & 6200 & No electncity was produced \\
\hline $\begin{array}{l}\text { Mobile Lower Power Plant No } 1 \\
\text { (DOE) }\end{array}$ & ML-1 & INEL Site, ID & $\begin{array}{l}\text { AGN } \\
\text { Gas cooled, light } \\
\text { water moderated }\end{array}$ & 3000 & 3,3000 & 6100 & 6500 & \\
\hline $\begin{array}{l}\text { Stationary Low Power Plant } \\
\text { No } 1 \text { (DOE) }\end{array}$ & SL-1 & INEL Site, ID & $\begin{array}{l}\text { ANL } \\
\text { Bolling water }\end{array}$ & 3000 & 2,2000 & 5800 & 6100 & \\
\hline
\end{tabular}

\section{B. Propulsion Experiments and Prototypes}

\begin{tabular}{|c|c|c|c|}
\hline $\begin{array}{l}\text { Alrcraft Reactor Expenment } \\
\text { (DOE) }\end{array}$ & ARE & Oak Ridge, TN & $\begin{array}{l}\text { ORNL } \\
\text { Molten salt }\end{array}$ \\
\hline $\begin{array}{l}\text { Expenmental Propulsion Test } \\
\text { Reactor (DOE) }\end{array}$ & TORY IIA & NTS, NV & $\begin{array}{l}\text { LLNL } \\
\text { Air cooled }\end{array}$ \\
\hline $\begin{array}{l}\text { Expenmental Propulsion Test } \\
\text { Reactor (DOE) }\end{array}$ & TORY IIC & NTS, NV & $\begin{array}{l}\text { LLNL } \\
\text { Aur cooled }\end{array}$ \\
\hline
\end{tabular}

$\begin{array}{rrrr}00 & 1,5000 & 5400 & 5400 \\ 00 & 150,0000 & 6000 & 6100 \\ 00 & 600,0000 & 6400 & 6400\end{array}$

$\begin{array}{clll}\begin{array}{c}\text { Heat Transfer Reactor } \\ \text { Expenment No 1 (DOE) }\end{array} & \text { HTRE-1 } & \text { INEL Site, ID } & \text { ANPD } \\ \begin{array}{c}\text { Heat Transfer Reactor } \\ \text { Experiment No 2 (DOE) }\end{array} & \text { HTRE-2 } & \text { INEL Site, ID } & \begin{array}{l}\text { ANPD } \\ \text { Air cooled }\end{array} \\ \begin{array}{c}\text { Heat Transfer Reactor } \\ \text { Expenment No 3 (DOE) }\end{array} & \text { HTRE-3 } & \text { INEL Site, ID } & \begin{array}{l}\text { ANPD } \\ \text { Air cooled }\end{array} \\ \begin{array}{c}\text { Submanne Advanced Reactor } \\ \text { Prototype }\end{array} & \text { S3G } & \text { West Milton, NY } & \text { GE } \\ \begin{array}{c}\text { Submanne Intermediate Reactor } \\ \text { Mark A (DOE) }\end{array} & \text { S1G } & \text { West Milton, NY } & \begin{array}{l}\text { Pressurized water } \\ \text { GE }\end{array} \\ \begin{array}{c}\text { Submanne Thermal Reactor } \\ \text { Facility (DOE) }\end{array} & \text { S1W } & \text { INEL Site, ID } & \begin{array}{l}\text { Sodum } \\ \text { West }\end{array} \\ \begin{array}{ll}\text { Pressunzed water } \\ \text { (D) }\end{array} & & & \end{array}$

20,0000

5600

5700

14,0000

5700

6100

00

32,0000

5800

6100

00

00

5800

9100

00

00

5500

5700

5300

Authonzed power not avalable

Authonzed power not avalable

The TORY IC was successfully tested at full design power dunng 5/64 Sub-sequent to cancellation of the Pluto program $7 / 1 / 64$, the reactor was placed in the Pluto disassembly building at NST for storage In 1974 the reactor was transferred to the NERVA disassembly area for disassembly

Faculity (DOE)

Authonzed power not avaulable 


\section{TEST AND RESEARCH}

\section{PART III MILITARY REACTORS}

\section{A. Test}

\begin{tabular}{|c|c|c|c|c|c|c|c|c|}
\hline Name (Owner) & Designation & Location & $\begin{array}{c}\text { Principal } \\
\text { nuclear } \\
\text { contractor. } \\
\text { Reactor type }\end{array}$ & $\begin{array}{c}\text { Power } \\
\text { capacity } \\
\text { net } \\
k W(e)\end{array}$ & $\begin{array}{l}\text { Authorized } \\
\text { power } \\
\text { kW(t) }\end{array}$ & $\begin{array}{c}\text { Initial } \\
\text { criticality } \\
\text { (yr mo) }\end{array}$ & $\begin{array}{l}\text { Shutdown } \\
\text { (Jr mo) }\end{array}$ & Comment \\
\hline $\begin{array}{l}\text { Aerospace Systems Test Reactor } \\
\text { (USAF) }\end{array}$ & ASTR & Fort Worth, TX & $\begin{array}{l}\text { Convair. } \\
\text { LWR }\end{array}$ & 0.0 & $10,000.0$ & 5400 & 7100 & $\begin{array}{l}\text { Defueled in } 1971 \text {; decommissioning } \\
\text { began in } 1973 \text { and was completed in } 1974 .\end{array}$ \\
\hline Ground Test Reactor (USAF) & GTR & Fort Worth, TX & Convair. Pool & 0.0 & $10,000.0$ & 5300 & 7300 & $\begin{array}{l}\text { Decommissioning began in } 1973 \text { and } \\
\text { was completed in } 1974 .\end{array}$ \\
\hline $\begin{array}{l}\text { Nuclear Engineering Test } \\
\text { Reactor (USAF) }\end{array}$ & NETR & Dayton, $\mathrm{OH}$ & $\begin{array}{l}\text { Maxon-AC. } \\
\text { Tank }\end{array}$ & 0.0 & $10,000.0$ & 6500 & 7000 & \\
\hline \multicolumn{9}{|l|}{ B. Research } \\
\hline $\begin{array}{l}\text { Army Materials Research Reac- } \\
\text { tor (USA). Regulated by NRC }\end{array}$ & AMRR & Watertown, MA & $\begin{array}{l}\text { BAC. } \\
\text { Pool }\end{array}$ & 0.0 & $5,000.0$ & 6000 & 7000 & Amy Materials and Mechanics Research Center. \\
\hline $\begin{array}{l}\text { Diamond Ordnance Radiation } \\
\text { Facility (USA) }\end{array}$ & DORF & Forest Glen, MD & $\begin{array}{l}\text { GA. } \\
\text { TRIGA-Mk F }\end{array}$ & 0.0 & 250.0 & 6100 & 7700 & Hamy Diamond Laboratories. \\
\hline $\begin{array}{l}\text { Naval Research Reactor (USN). } \\
\text { Regulated by NRC }\end{array}$ & NRR & Washington, D.C. & $\begin{array}{l}\text { NRL. } \\
\text { Pool }\end{array}$ & 0.0 & $1,000.0$ & 5600 & 7000 & \\
\hline Nuclear Effects Reactor (DOE) & Super KUKLA & NTS, NV & LLNL. Prompt burst & 0.0 & 0.0 & 6400 & 7900 & Standby fuel in storage at $\mathrm{ORNL}$. \\
\hline $\begin{array}{l}\text { Thermal Test Reactor No. } 1 \\
\text { (DOE) }\end{array}$ & TTR-1 & Schenectady, NY & $\begin{array}{l}\text { KAPL. } \\
\text { Graphite }\end{array}$ & 0.0 & 10.0 & 5100 & 8300 & \\
\hline $\begin{array}{l}\text { Walter Reed Research Reactor } \\
\text { (USA). Regulated by NRC }\end{array}$ & WRRR & Washington, D.C. & $\begin{array}{l}\text { AI. } \\
\text { Homogeneous }\end{array}$ & 0.0 & 50.0 & 6200 & 7000 & Walter Reed Army Institute of Research. \\
\hline
\end{tabular}

\section{POWER REACTORS}

\section{PART IV EXPORT REACTORS}

\section{A. Central-Station Electric Power Plants}

\section{Reactor name. Owner}

Location

France. Franco-Belgian Society for Nuclear

Energy of Ardennes, SENA.

Germany, Kahl Nuclear Power Station. Rhine-

Westphalia Power Co., RWE

Germany, Kemdraftwerk-RWE-Bayemwerk,

KRBI.

Italy, Garigliano Nuclear Power Station. Project

$$
\text { ENEL of SENN }
$$

Japan, Japan Power Demonstration Reactor. JAERI

\begin{tabular}{|c|c|c|}
\hline Location & $\begin{array}{c}\text { NRC } \\
\text { export } \\
\text { license No. } \\
\text { and date }\end{array}$ & $\begin{array}{l}\text { Principal } \\
\text { nuclear } \\
\text { contractor. } \\
\text { Reactor type }\end{array}$ \\
\hline Chooz & & $\begin{array}{l}\text { West./Fram., ACEC. } \\
\text { Pressurized water }\end{array}$ \\
\hline Kahl-am-Main & $\begin{array}{l}\text { XR-031 } \\
09 / 30 / 59\end{array}$ & $\begin{array}{l}\text { GE. } \\
\text { Boiling water }\end{array}$ \\
\hline $\begin{array}{l}\text { Gundremmingen } \\
\text { (near Gunzburg) }\end{array}$ & $\begin{array}{l}\text { XR-052 } \\
05 / 28 / 64\end{array}$ & $\begin{array}{l}\text { GE. } \\
\text { Boiling water }\end{array}$ \\
\hline $\begin{array}{l}\text { Punta Fiume (on } \\
\text { Garigliano River) }\end{array}$ & $\begin{array}{l}\text { XR-043 } \\
08 / 16 / 61\end{array}$ & $\begin{array}{l}\text { GE. } \\
\text { Boiling water }\end{array}$ \\
\hline $\begin{array}{l}\text { Tokai-Mura, } \\
\text { Ibaraki Pref. }\end{array}$ & $\begin{array}{l}\text { XR-045 } \\
03 / 16 / 62\end{array}$ & $\begin{array}{l}\text { GE. } \\
\text { Boiling water }\end{array}$ \\
\hline
\end{tabular}

Power

design

MW(e)

Power

Author- Initial

ized critt.

power cality

$k W(t) \quad(y r m o)$

Shut-

MW(t)

6610

(yr mo)

Comment

15.6

0.0

6100

9110

Decommissioning in process.

801.0

150.0

506.0

$6400 \quad 7800$

12.0 


\section{B. Propulsion}

Great Bntain, S5W for HMS DREADNOUGHT

\section{TEST, RESEARCH, and TEACHING}

\section{A. General Irradlation Test}

(No reactors in this category)

\section{B. General Research}

$\overrightarrow{4}$

Reactor name. Owner

Belgium, BR 3, Mol AMITAS

Denmark, DR-2 Riso Natuonal Laboratory

Germany, FRG-1 GKSS-Forschungszentrum Geesthach $\mathrm{G}$ m b H

Italy Center for Multary Application of

Nuclear Energy

Italy European Community Commission

Italy Fiat TTG

Italy Itahan Agency for New Technology, Energy and the Environment

Japan Japan Atomic Energy Research Instutute

Vıtnam Vietnam Instutute of Nuclear Research

West Berlin, City of Instutute for Nuclear

Research
West

Pressunzed water

00

00

00

6200

0000

Westunghouse was the designer of the reactor Designation S5W No power levels avaulable As of $2 / 90$ the reactor has been

defueled and is not in

operational condition

\begin{tabular}{|c|c|c|c|c|c|c|c|c|}
\hline Location & $\begin{array}{c}\text { NRC } \\
\text { export } \\
\text { license No } \\
\text { and date }\end{array}$ & $\begin{array}{l}\text { Principal } \\
\text { nuclear } \\
\text { contractor. } \\
\text { Reactor type }\end{array}$ & $\begin{array}{c}\text { Power } \\
\text { design } \\
\text { net } \\
\text { MW(e) }\end{array}$ & $\begin{array}{l}\text { Power } \\
\text { MW(t) }\end{array}$ & $\begin{array}{l}\text { Author- } \\
\text { ized } \\
\text { power } \\
\text { kW(t) }\end{array}$ & $\begin{array}{l}\text { Initial } \\
\text { critl- } \\
\text { cality } \\
\text { (yr mo) }\end{array}$ & $\begin{array}{l}\text { Shut- } \\
\text { down }\end{array}$ & Comment \\
\hline Mol & $\begin{array}{l}\text { XR } 024 \\
11 / 06 / 58\end{array}$ & Pressunzed water & 105 & 00 & 00 & & & $\begin{array}{l}\text { The reactor contains some fuel and } \\
\text { is sull under IAEA junsdiction }\end{array}$ \\
\hline Riso & $\begin{array}{l}\text { XR } 006 \\
04 / 04 / 57\end{array}$ & $\begin{array}{l}\text { FW } \\
\text { Tank }\end{array}$ & 00 & 00 & 50000 & 5800 & 7500 & \\
\hline Geesthacht & $\begin{array}{l}\text { XR 008 } \\
06 / 10 / 57\end{array}$ & $\begin{array}{l}\text { B\&W Research } \\
\text { Reactor }\end{array}$ & 00 & 00 & 50000 & 5810 & nd & \\
\hline Near Pisa & $\begin{array}{l}\text { XR-036 } \\
12 / 17 / 59\end{array}$ & $\begin{array}{l}\text { B\&W } \\
\text { Pool }\end{array}$ & 00 & 00 & 00 & 6300 & 8000 & Power Pulsung \\
\hline Ispra & $\begin{array}{l}\text { XR-007 } \\
05 / 17 / 57\end{array}$ & $\begin{array}{l}\mathrm{AC} \\
\text { Heavy water, tank }\end{array}$ & 00 & 00 & 50000 & 5900 & 8900 & \\
\hline Saluggia & $\begin{array}{l}\text { XR-016 } \\
12 / 06 / 57\end{array}$ & $\begin{array}{l}\text { AMF } \\
\text { Pool }\end{array}$ & 00 & 00 & 70000 & 5900 & 7300 & \\
\hline Casaccia & $\begin{array}{l}\text { XR-051 } \\
04 / 20 / 64\end{array}$ & $\begin{array}{l}\text { AMF } \\
\text { Pool }\end{array}$ & 00 & 00 & 00 & 7100 & 7900 & Negligible power \\
\hline $\begin{array}{l}\text { Tokar-Mura, } \\
\text { Ibarak1 Pref }\end{array}$ & $\begin{array}{l}\text { XR-001 } \\
11 / 02 / 56\end{array}$ & $\begin{array}{l}\text { AI } \\
\text { L-54 }\end{array}$ & 00 & 00 & 500 & 5700 & 6900 & \\
\hline Dalat & $\begin{array}{l}\text { XR 032 } \\
10 / 10 / 59\end{array}$ & $\begin{array}{l}\text { GA } \\
\text { TRIGA Mk II }\end{array}$ & 00 & 00 & 2500 & 6300 & 7300 & \\
\hline West Berlun & $\begin{array}{l}\text { XR } 012 \\
09 / 07 / 57\end{array}$ & AI L 54 & 00 & 00 & 500 & 5800 & 7000 & \\
\hline
\end{tabular}


Germany Universittes of Frankfurt and Darmstadt

Italy University of Mulan

$\begin{array}{lll}\text { Frankfun } & \text { XR 009 } & \text { AI } \\ & 05 / 01 / 57 & \text { L-54 } \\ \text { Milan } & \text { XR-022 } & \text { AI } \\ & 08 / 13 / 58 & \text { L-54 } \\ \text { Geneva } & & \text { AGN } \\ & & \text { AGN-201 111 }\end{array}$

London

United Kingdom Queen Mary College, London University

Switzerland University of Geneva

$\begin{array}{ll}\text { XR-049 } & \text { AS Inc } \\ 02 / 13 / 64 & \text { UTR-B }\end{array}$

\section{TEST, RESEARCH, and TEACHING}

\section{Unlversity Research and Teaching}

$\begin{array}{llll}\text { Reactor name. Owner } & \text { Location } & \begin{array}{c}\text { NRC } \\ \text { export } \\ \text { license No. } \\ \text { and date }\end{array} & \begin{array}{c}\text { Principal } \\ \text { nuclear } \\ \text { contractor. } \\ \text { Reactor type }\end{array} \\ \text { Germany Association fer Radiation Research } & \text { Munich } & \begin{array}{l}\text { XR-075 } \\ 01 / 14 / 71\end{array} & \begin{array}{l}\text { GA } \\ \text { TRIGA Mk III }\end{array} \\ \text { Germany Brown Boven/Krupp } & \text { Julich } & \begin{array}{l}\text { XR-062 } \\ 07 / 28 / 66\end{array} & \text { AI } \\ \text { L-77A }\end{array}$

\section{PART IV EXPORT REACTORS}

$\begin{array}{ccccc}\begin{array}{c}\text { Power } \\ \text { design }\end{array} & & \begin{array}{c}\text { Author- } \\ \text { Ized }\end{array} & \begin{array}{c}\text { Initial } \\ \text { critl- }\end{array} \\ \text { net } & \text { Power } & \begin{array}{c}\text { power } \\ \text { cality }\end{array} & \text { Shut- } \\ \text { MW(e) } & M W(t) & \text { kW(t) } & (y r \text { mo) } & \text { down }\end{array}$

00

00

00

10000

00

00

6400 Sterkrade A E Thasland, 11/62

was sold through Gulf

L $L-77$ reactor was operated in the commencal exhibit of the 1958 International Conference in Geneva and in the USAEC Atoms for Peace Exhibits in Beinut, Lebanon, 10/61, in Athens, Greece, 5/62, and in Bangkok,

Negligible power The AGN-201111 was operated first in the USAEC Atoms for Peace Exhibit in Rome, Italy, 7/58 and later in the commercial exhibit of the 1958 International Conference in

Geneva prior to transfer to the Unversity of Geneva

\section{CIVILIAN}

\section{PART V CRITICAL ASSEMBLIES}

$\begin{gathered}\text { Initial } \\ \text { criticality } \\ \text { (yr mo) }\end{gathered}$
6300

Facility (Regulatory agency)
Argonne Natuonal Laboratory (DOE)

$\begin{array}{cr}\text { Designation } & \text { Location } \\ \text { ZPR-6 } & \text { Argonne, IL }\end{array}$

\section{Equjpment}

\begin{tabular}{cc}
\hline $\begin{array}{c}\text { No. of } \\
\text { of }\end{array}$ & $\begin{array}{c}\text { control } \\
\text { panels } \\
\text { room }\end{array}$ \\
2 & 2
\end{tabular}

Abbreviation

ANL

\section{Subject of experiment or study. Comment \\ Shut- \\ down \\ (yr mo)}

8200 Basic fast reactor studies and mock-up for LMFBR 
ANL power expenments of histoncal interest previously conducted in ANL facility cells include the NAUTILUS core design (ZPR-1), the Savannah River reactor design (ZPR-2), and a senes of fast-neutron studes (ZPR-4) and interactions between two basic systems (ZPR-5) The following experments were performed in the ZPR-7

facility thonum, uranium, deutenum criticals (THUD), and a senes of flux-trap cnticals for the Argonne High Flux Research Reactor

Bett1s Atomic Power Laboratory (DOE) Cntucal Facilty-10, Lynchburg Research Center (NRC)

Los Alamos Natuonal Laboratory (DOE)

Los Alamos National Laboratory (DOE)

PNL Critucal Mass Laboratory (DOE)

$\begin{array}{lll}\text { LWBCC } & \text { Pittsburgh, PA } & 3 \\ \text { SSRF } & \text { Lynchburg, VA } & 2 \\ & & \\ \text { Parka } & \text { Los Alamos, NM } & 3 \\ \text { Venus } & \text { Los Alamos, NM } & 1\end{array}$

\section{Betus}

CX-10

6300

8000

LWB physics

LANL, Kiva III $\quad 6300$

LANL, Kiva I $\quad 7600$

PNL-CML

7600

Honzontal

RSTM

Hanford Site, WA Hanford Site, WA Pawling, NY

PNL-CML

PNL-CML

UNC

6100

6300

6700
Cold critical for instrumentation testung Defueled

8800 Verucal table assembly machine

9000 The Critucal Mass Laboratory was used to expenment with laquid plutonium solutions It was shut down in 1990 and transferred to the surplus faclintes program for

decontamination and decommissioning

9000 See FEAS reactor

9000 See FEAS reactor

7200 Proff test faculity Material license (SNM-871) termınated $7 / 14 / 75$

\section{MILITARY}

\section{Facility (Regulatory agency)}

Betus Atomic Power Laboratory (DOE) Betts Atomic Power Laboratory (DOE) Knolls Atomic Power Laboratory (DOE) Knolls Atomic Power Laboratory (DOE) Lockheed Aurcraft Co , Cnucal Faculty for RER (NRC)

\begin{tabular}{ll} 
Designation & \multicolumn{1}{c}{ Location } \\
HTTF & Pitsburgh, PA \\
SS-CF & Pitsburgh, PA \\
FPR & Schenectady, NY \\
PTR & Schenectady, NY \\
CERF & Dawsonville, GA
\end{tabular}

Equipment

$\begin{array}{cc}\text { No. of } \\ \text { of } & \text { control } \\ \text { cells } & \text { panels' } \\ & \text { room }\end{array}$

room

0

0
0
0

$\begin{array}{lc}\text { Abbreviation } & \begin{array}{r}\text { Initial } \\ \text { criticalit } \\ (\text { yr mo) }\end{array} \\ \text { Bettus } & 5900 \\ \text { Betus } & 5700 \\ \text { KAPL } & 5600 \\ \text { KAPL } & 5800 \\ \text { Lockheed } & 5800\end{array}$

Shut-

down

(yr mo)

\section{Subject of experimen \\ or study. Comment}

$8400 \quad$ Surface-ship physics

7600 Surface-ship physics

7500 Flexible cntical expenments

7600 High-temperature high-pressure physics and mock-up 
REACTOR INDEX 
A1W, 14

Aberdeen Pulsed Reactor Facilıty, 15 ABRAHAM LINCOLN

CVN72, 13

SSBN602, 42

Accelerator Pulsed Fast Cnucal Assembly, 33

ACPR, 33

ACRR, 9

Advanced Neutron Source Reactor, 9

Advanced Reactıvity Measurement Facilty, 8

Advanced Test Reactor, 8

Advanced Test Reactor Crutul Facilty, 22

Aerospace Systems Test Reactor, 44

Aerotest Operations, Inc , 9

AFRRI, 15

AFSR, 9

AGNIR, 9

Aurcraft Reactor Expenment, 43

ALABAMA, 12

ALASKA, 12

Albania, 20

ALBANY, 13

ALBUQUEROUE, 12

ALEANDER HAMILTON, 12

ALEXANDRIA, 13

ALRR, 31

Alvin W Vogule Nuclear Plant

Unit 1,1

Unit 2,1

Amencan Standard Inc , 33

Ames Laboratory Research Reactor, 31

AMRR, 44

ANDREW JACKSON, 42

ANNAPOLIS, 13

Annular Core Pulsed Reactor, 33

ANS, 9

APFA-III, 33

APRF, 15

ARCHERFISH, 13

ARE, 43

Argonaut (CP-11), 33

Argonne CP-3, rebult as CP-3', 33

Argonne Low Power Research Reactor, 33

Argonne National Laboratory

AGN-201-108, 33

Idaho Division, 22

ZPR-6, 46

ZPR $-9,47$

Argonne Nuclear Assembly for University

Trainung, 33

Argonne Research Reactor, 31
Argonne Thermal Source Reactor, 33

Anzona, University of,

ARKANSAS (2 reactors), 13

Arkansas Nuclear One

Unit 1, 1

Unit 2,1

Arkansas Tech University, 11

Armed Forces Radıbiology Research

Instutute, 15

ARMF, 8

Army Matenals Research Reactor, 44

ARR (L-54), 35

ASHEVILLE, 13

ASPRO, 12

ASTR, 44

ATLANTA, 12

Atomics Intemational, 33

ATR, 8

ATRC, 22

ATSR, 33

AUGUSTA, 12

Australıa, Moata, 19

Austria

Astra, 19

Vienna Polytechnic Insutute, 21

B Reactor, 40

Babcock \& Wilcox Lynchburg Pool Reactor, 33

Babcock \& Wilcox Nuclear Development

Center Test Reactor, 31

BAINBRIDGE (2 reactors), 13

BALTTMORE, 12

Bangladesh, 19

BARB, 42

BATFISH, 13

BATON ROUGE, 13

Battelle Memorial Insutute, 33

BAWTR, 31

Beaver Valley Power Station

Unit 1, 1

Unit 2,1

Belgium

BR-3, Mol, 45

Doel

Unit 1,15

Unit 2, 15

Unit 4, 15

Tihange

Unit 1,15

Unit 3,15
Bellefonte Nuclear Plant

Unit 1,6

Unt 2.6

BENJAMIN FRANKLIN, 12

BERGALL, 13

Bettus Atomic Power Laboratory

HTTF, 47

LWBCC, 47

SS-CF, 47

BGRR, 33

Big Rock Point Nuclear Plant, 1

Big Ten, 22

BILLFISH, 13

Biological Research Reactor, 9

BIRMINGHAM, 13

BLUEFISH, 13

BMRR 8

BNL/FS-1, 34

Bollng Nuclear Superheater Power Station, 23

Bollung Reactor Expenments, 25

BOISE, 13

BORAX reactors, 25

BOSTON, 12

Braidwood Station

Unit 1,1

Unit 2,

Brazl

Angra 1, Central Electncia de Fumas, 15

Minas Gerars, University of, 21

São Paulo, University of, 21

BREMERTON, 13

Brigham Young University, 37

Brookhaven Graphite Research Reactor, 33

Brookhaven High Flux Beam Research Reactor,

Brookhaven Medical Research Reactor, 8

Brookhaven Neutron Source Reactor

No 1,33

No 2, 34

Browns Ferry Nuclear Power Station

Unit 1, 1

Unit 2, 1

Unit 3, 1

BRR, 33

Brunswick Steam Electric Plant

Unit 1, 1

Unit 2,1

BSR, 34

BUFFALO, 12

Bulk Shieldıng Reactor, 34
Byron Station

Unit 1,1

Unit 2.1

C Reactor

Richland, 40

Savannah River, 11

CALIFORNIA (2 reactors), 13

Calıfomia

Berkeley, University of, 37

Irvine, University of, 9

Los Angeles, University of, School of Engineerning and Applied Science, 37

Santa Barbara, University of, 37

Calıfomıa Polytechnıc State University, 37

Callaway Plant, Unit 1, 1

Calvert Cliffs Nuclear Power Plant

Unit 1,1

Unit 2, 2

Canada, 21

CARL VINSON (2 reactors), 13

Carolınas Vırgma Tube Reactor, 23

CASIMIR PULASKI, 12

Catawba Nuclear Station

Unit 1,2

Unit 2,2

Catholıc University of America, 37

CAVALIER, 39

CAVALLA, 13

CEF, 22

CERF, 47

CFRMF, 9

CHARLOTTE, 13

HEYENNE, 14

CHICAGO, 12

Chicago Ple 1, rebult as CP-2, 34

China, Republic of, 21

CINCINNATI, 13

CLNR, 3

Cinuchem, Inc Reactor, 31

CITY OF CORPUS CHRISTI, 12

Clemenune, 35

Clinton Power Station, Unit 1,2

Colombia, IAN-R1, 19

Colorado State Unversity, 37

COLUMBIA, 14

Columbia University, 38

COLUMBUS, 13

Comanche Peak Stearn Electrc Station Unit 1,2

Unit 2,6 
Comet, 22

Cooper Nuclear Station, 2

Comell University, 9

Comell University Zero Power Reactor, 9

Coupled Fast Reactuvity Measurement Fachlty, 9

CP-2, 34

CP-3', 33

CP-5, 31

Crncal Faclity-10, Lynchburg Research Center, 47

Crystal Ruver Nuclear Plant, Unit 3, 2

Curtuss-Wright Nuclear Research Laboratory of

the Commonwealth of Pennsylvanua, 34

CWRR, 34

$\mathrm{CX}, 22$

D Reactor, 40

D1G, 14

DACE, 42

DALLAS, 13

DANIEL BOONE, 12

DANIEL WEBSTER, 12

Davis-Besse Nuclear Power Station, Unit 1,2

Deep Submergence Research Vehicle, 13

in Delaware, University of, 38

Demo Reac, 34

Denmark

DR-1, 19

DR-2, 45

Destroyer Reactor Prototype, 14

Diablo Canyon Nuclear Power Plant

Unit 1, 2

Unit 2, 2

Dramond Ordnance Radıatıon Facilty, 44

DOE Demonstration Reactor, 34

Donald C Cook Nuclear Power Plant

Unit 1,2

Unit 2,2

DORF, 44

Dow Chemical Co 9

DR Reactor, 40

Dresden Nuclear Power Station

Unit 1, 23

Unit 2, 2

Unit 3,2

DRUM, 13

Duane Amold Energy Center, Unit 1, 2

DWIGHT D EISENHOWER (2 reactors), 13

EAEP, 34

EBOR, 25
FRAN, 36

France, 44

FRANCIS SCOTT KEY, 13

Fuel Element Test Bed, 28

Fuel Element Test Reactor

Pewee-1, 28

Pewee-2, 28

Gas Cooled Reactor Expenment, 43

GATO, 12

GCRE, 43

General Atomics

Advanced TRIGA-Mk F Prototype Reactor, 9

TRIGA-Mk I Prototype Reactor, 9

General Atomics Technologies, 34

General Electnc Nuclear Test Reactor, 9

General Electnc Testung Reactor, 30

GEORGE BANCROFT, 12

GEORGE C MARSHALL, 13

GEORGE WASHINGTON

CVN73, 14

SSN598, 42

GEORGE WASHINGTON CARVER, 13

GEORGIA, 12

Georgia Institute of Technology

AGN-201-104, 38

GTRR, 9

Germany

Association for Radiation Research, 46

Brown Bovern/Krupp, 46

FRG-1, 45

FRM Gersching, 21

Insturute for Nuclear Medicine, 21

Johannes Gutenberg University of Mainz, 21

Kahl Nuclear Power Station, 44

Kemkraftwerk-RWE-Bayemwerk, KRBI, 44

Medical College of Hanover, 21

Mulheim-Kaerhch, 15

Universitues of Frankfurt and Darmstadt, 46

GETR, 30

GLENARD P LIPSCOMB, 42

Godiva-IV, 22

Grand Gulf Nuclear Station, Unit 1, 2

GRAYLING, 12

Great Britain, S5W for HMS

DREADNOUGHT, 45

Greece, Democntos, 19

GREENLING, 12

GREENVILLE, 14

GROTON, 13
Humboldt Bay Power Plant, Unit 3, 23

HWCTR, 26

HYMAN G RICKOVER, 12

HYPO, 35

Idaho State University, 9

Illınor Institute of Technology Research Instutute, 35

Illinots, University of

LOPRA, 10

TRIGA-Mk I, 10

India

Tarapur Nuclear Power Station

Unit 1,15

Unit 2,15

Indıan Point Station

Unit 1, 23

Unit 2,3

Unit 3,3

INDIANAPOLIS, 13

Indones1a

Bandung, 19

Yogyakarta, 19

Industnal Reactor Laboratones, Inc , 31

lowa State University, 10

Iran, 21

IRL, 31

Israel, 19

Italy

Caorso Nuclear Statuon, 15

Center for Military Application of Nuclear

Energy, 45

European Community Commission, 45

Frat TTG, 45

Ganghano Nuclear Power Station, 44

Italıan Agency for New Technology, Energy and the Environment

Casaccia, 45

Rome, 19

Milan, University of, 46

Palermo, University of, 2

Pavia, Universily of, 2

Trno Vercellese, 15

JACK, 42

JACKSONVILLE, 13

Jamaica, 19

James A FitzPatnck Nuclear Power Plant, 3

JAMES K POLK, 12

JAMES MADISON, 12
Kiw1-Transient Test Reactor, 31

Kiw1-TTR, 31

Knolls Atomic Power Laboratory

FCPE, 22

FPR, 47

PTR, 47

Korea

Advanced Energy Research Instutute

XR-027, 19

$\mathrm{XR}-073,19$

Kon-1, 16

Kon-2, 16

Kon-3, 16

Kon-4, 16

Kyung Hee, University of, 2

Ulchin 3,18

Ulchin 4, 18

Yonggwang-1, 16

Yonggwang-2, 16

Yonggwang-3, 17

Yonggwang-4, 17

KUKLA, 36

KW Reactor, 40

L Reactor, 11

$\mathrm{L}-47,33$

$\mathrm{L}-77,37$

L-85 (AE 6), 36

L. MENDELL RIVERS, 13

a Crosse (Genoa) Nuclear Generating Staton, 23

LA JOLLA, 13

La Salle County Station

Unit 1,3

LAFAYETTE, 42

LAMPRE-1, 26

LAPON, 13

LAPRE-1, 26

LAPRE-2, 27

Large Shyp Reactor Prototype (2 reactors), 14

Lattice Test Reactor, 40

Leland Stanford University, 38

LEWIS AND CLARK, 12

Limenck Generatung Statıon

Unit 1,3

Unit 2,3

LITR, 35

Livermore Pool Type Reactor, 35

L.uvermore Water Boller, 35

LWWB, 35 
Ground Expenmental Engine Expenment XE-Backup, 28

Educator, 37

Edwin I Hatch Nuclear Plant

Unit 1,2

Unit 2, 2

EGCR, 26

Elk River Reactor, 23

Enguneenng Test Reactor, 30

England

Impenal Chemical Industries, 19

Sizewell B, 17

Ennco Fermı Atomic Power Plant

Unit 1,23

Unit 2, 2

ENTERPRISE (8 reactors), 13

EOCR, 26

ESADA Vallectos Expenmental Superheat Reactor, 25

ETHAN ALLEN, 42

ETR, 30

European-Asıan Exhıbit Program, 34

EVESR, 25

Experimental Beryllium Oxıde Reactor, 25

Experimental Boulng Water Reactor, 25

Expenmental Breeder Reactor II, 7

Experumental Breeder Reactor No 1,26

Expenmental Gas Cooled Reactor, 26

Expenmental Organic Cooled Reactor, 26

Expenmental Propulsion Test Reactor

TORY IIA, 43

TORY IIC, 43

F Reactor, 40

Fast Burst Reactor Faculty, 15

Fast Flux Test Faclity, 8

Fast Neutron Source Reactor, 34

Fast Source Reactor, 9

FBRF, 15

FCPE, 22

FEAS, 47

FFTF, 8

FINBACK, 13

Finland, 21

FLASHER, 12

Flattop, 22

FLORIDA, 12

Flonda, University of, 9

FLYING FISH, 13

FNR, 10

Fort Calhoun Station, Unt 1, 2

Fort St Vrain Nuclear Generatung Station, 23 FPR, 47
XE-Prime, 29

Ground Test Reactor, 44

GTR, 44

GTRR, 9

GUARDFISH. 12

GUITARRO, 13

GURNARD, 13

H Reactor, 40

H B Robinson Plant, Untt 2, 3

Haddam Neck Plant, 3

HADDO, 42

HADDOCK, 12

HALIBUT, 42

Hallam Nuclear Power Facility, Sheldon Stanon, 23

HAMMERHEAD, 13

HAMPTON, 14

Hanford 305 Test Reactor, 40

HARTFORD, 14

HAWKBILL, 13

Health Physics Research Reactor, 34

Heat Transfer Reactor Expenment

No 1,43

No 2,43

No 3,43

Heavy Water Components Test Reactor, 26

HELENA, 12

HENRY CLAY, 42

HENRY L STIMSON, 13

HENRY M JACKSON, 12

HFBR, 8

HFIR, 8

High Flux Isotope Reactor, 8

High Temperature Latuce Test Reactor, 35

Homogeneous Reactor Expenment

No 1,26

No 2,26

Honeycomb, 22

HONOLULU, 12

Honzontal, 47

Honzontal/Splıt Table, 22

HOUSTON, 12

HPRR, 34

HRE-1, 26

HRE-2, 26

HTLTR, 35

HTR, 40

HTRE-1, 43

HTRE-2, 43

HTRE-3, 43

HTTF, 47
JAMES MONROE, 42

JANUS, 9

Fukushima Dat ichi Power Station

Unit 1,15

Unit 2,15

I Iñt 6,16

Japan Atomic Energy Research Institute, 45

Japan Power Demonstration Reactor, 44

JRR-2, 18

Kashwazakı-Kanwa

Unit 6, 17

Kınkı University, 21

Mihama Power Station, Unit 1, 16

Musashı College of Technology, 2

NSRR, 18

Ohı Power Station

Unit 1,16

Unit 2, 16

Rukkyo Unuversity, 21

Takahama Power Station, Unit 1, 16

Tokat No 2 Power Statıon, 16

Tsuruga Power Station, Unit 1, 16

JEFFERSON CITY, 13

JOHN ADAMS, 42

JOHN C CALHOUN, 12

JOHN C STENNIS, 14

JOHN MARSHALL, 12

Joseph M Farley Nuclear Plant

Unit 1,3

Unit 2, 3

Juggemaut, 33

K Reactor, 11

KAMEHAMEHA, 12

Kansas, University of, 38

Kansas State Unuversity, 10

KE Reactor, 40

KENTUCKY, 13

Kewaunee Nuclear Power Plant, 3

KEWB, 35

KEY WEST, 12

Kunetuc Expenment on Water Bollers, 35

King Intense Neutron Generator, 3

Kunglet, 31

Kıw1-A, 29

Kiwı A Prime, 29

Kiwl-A3, 29

Kıw1-B1A, 29

Kiwi-B1B, 29

Kiw1-B4A, 29

$K_{1 w_{1}-B 4 D, 29}$

Kıwı-B4E, 30
Lockheed Aircraft Co., Cntical Facilty for RER, 47

Lockheed Aurcraft Corp , 35

LOFT, 32

LONG BEACH (2 reactors), 13

LOPO, 35

LOPRA, 10

Los Alamos Fast Reactor, 35

Los Alamos LOPO Reactor, 35

Los Alamos Molen Plutonium Reactor

Experiment, 26

Los Alamos National Laboratory

Big Ten, 22

Comet, 22

Flattop, 22

Godiva-IV, 22

Honeycomb, 22

Mars, 22

Parka 47

Planet, 22

SHEBA, 22

SKUA, 22

Venus, 47

Los Alamos Power Reactor Expenment

No 1,26

Los Alamos Water Boller

HYPO, 35

SUPO, 35

LOS ANGELES, 13

Loss of Flu1d Test, 32

Louisiana State University Nuclear Science Center, 35

LOUISVILLE, 12

Low Intensity Test Reactor, 35

Low Temperature Neutron Irradiation Faclity, 35

Lowell, Unıversity of, 10

LPTR, 35

LRP, 33

LTNIF, 35

LTR, 40

LWBCC, 47

MAINE, 13

Mane Yankee Atomic Power Plant, 3

Malaysia, 19

Manhattan College, 10

MARF, 14

MARIANO G VALLEJO, 13

Mars, 22

MARYLAND, 13

Maryland, University of, 10

Massachusetts Insutute of Technology, 10

Materials Testung Reactor, 30

MCZPR, 10 
MEMPHIS, 13

Memphis State Unıversity, 38

Mexico

Laguna Verde Station, Unit 1, 16

Laguna Verde Station, Unt 2, 17

National Commission for Nuclear Energy, 19

MH-1A, 41

MIAMI, 13

MICHIGAN, 12

Michıgan, Universtty of (Ford Nuclear Reactor), 10

Michigan State University, 38

Millstone Nuclear Power Station

Unit 1,3

Unit 2,3

Unit 3,3

MINNEAPOLIS-SAINT PAUL, 12

MISSISSIPPI (2 reactors), 13

Mississippi State University, 38

Missoun, University of, 10

Missoun at Rolla, University of, 10

MITR-II, 10

ML-1, 43

Mobile Low Power Plant No 1, 43

Model 4180, 38

Modıficatuons and Addutuons to Reactor Facllty, 14

Modular High Temperature Gas Reactor, 11

Molten Salt Reactor Expenment, 27

Montucello Nuclear Generating Plant, 3

MONTPELIER, 13

Morocco, 20

MSRE, 27

MTR, 30

MUR, 35

MURR, 10

N Reactor, 24

NARWHAL, 13

NASA Mock-Up Reactor, 35

NASA-TR, 30

NATHAN HALE, 42

NATHANAEL GREENE, 42

Natuonal Instutute of Standards \& Technology, 8

Natural Circulation Test Reactor, 14

NAUTILUS, 42

Naval Research Reactor, 44

NEBRASKA, 13

Netherlands

Delft Technical University, 21

Dodewaard, GKN, 16

Enerov Center 18
Kiw1-B4D, 29

Kiw1-B4E, 30

Phoebus 1A, 30

Phoebus 1B, 30

Phoebus 2A, 30

Nuclear Safety Factlity, Rocky Flats Plant

Honzontal/Split Table, 22

Solution Base, 22

Vertucal/Splt Table, 22

Water Reflector Tank, 22

Nuclear Shıp SAVANNAH, 25

Oak Ridge Critical Experiments Facility, 22

Oak Ridge Graphite Reactor, 36

Oak Ridge Research Reactor, 31

Oconee Nuclear Station

Unt 1, 3

Unit 2, 3

Unit 3,3

OHIO, 12

Ohio State University, 10

Oklahoma, University of, 39

OKLAHOMA CITY, 12

OLYMPIA, 12

OMAHA, 13

Omaha Veterans Adminıstration Hospital, 9

Omega West Reactor, 8

OMRE, 27

Oregon State University

AGN-201-114, 39

OSTR, 10

ORG, 36

Organic Moderated Reactor Experiment, 27

ORNL Pool Cnucal Assembly, BSF Pool, 22

ORR, 31

OSTR, 10

OSURR, 10

OWR, 8

Oyster Creek Nuclear Power Plant, Unt 1, 4

P Reactor, 11

Pakistan, PARR, 19

Palisades Nuclear Plant, Unit 1,4

Palo Verde Nuclear Generating Station

Unit 1, 4

Unit 2, 4

Unit 3, 4

PARCHE, 13

PARGO, 13

Parka, 47

PASADENA 13
PORTSMOUTH, 12

Portugal, RP-1, 20

Power-Burst Facilty, 8

Praune Island Nuclear Generatung Plant

Unit 1, 4

Unit 2,4

Process Development P1le, 40

PROVIDENCE, 12

$\mathrm{PRR}, 36$

PRTR, 27

PSTR, 10

PTF, 47

PTR, 47

Pueno Rico Nuclear Center

L-77, 39

L-77, 39

TRIGA FLIP, 39

PUFFER, 13

PULSTAR, 10

PUR-1, 10

Purdue University, 10

Quad-Cities Station

Unit 1, 4

Unut 2,4

QUEENFISH, 42

R Reactor, 40

Radıatuon Effects Reactor, 36

Rancho Seco Nuclear Generatung Station, Untt 1, 24

RAY, 13

Reed College, 10

Rensselaer Polytechnic Institute, Troy, NY, 22 RER 36

RHODE ISLAND, 13

Rhode Island Nuclear Science Center, 9

RICHARD B RUSSELL, 13

RINSC 9

River Bend Station, Unit 1, 4

ROBERT E LEE, 42

Robert Emmet Ginna Nuclear Power Plant,

$$
\text { Unit } 1,4
$$

Rockwell Intemational, 36

Romania, Instutute for Nuclear Technologies, 18, 19

RRR, 38

RSTM 47

S1OFS-1, 28

S10FS-3, 28
Shoreham Nuclear Power Station, 24

SILVERSIDES, 13

SIMON BOLIVAR, 12

SKATE, 42

SKIPJACK, 42

SKUA, 22

SL-1, 43

SM-1, 41

SM-1A, 41

Small Submanne Reactor Prototype, 14

SNAP 02 Developmental System 27

SNAP-02 Experimental Reactor, 27

SNAP-02/10A TSF Shielding Experiment, 27

SNAP-08 Developmental Reactor, 28

SNAP-08 Expenmental Reactor, 28

SNAP-10A Flight System Ground Test

No 1,28

No 3,28

SNAP-10A Flight System

S10FS-4, 28

S10FS-5, 28

SNAP-10A Transient Test

No 2,32

No 3,32

SNAP-TSF 27

SNAPTRAN-2, 32

SNAPTRAN $-3,32$

SNARE, 35

SNL Annular Core Research Reactor, 9

SNL Critucal Assembly, 22

SNOOK, 42

SNRS-1, 15

Sodium Reactor Expenment, 27

Solution Base, 22

South Afnca, Safan-1, 19

SOUTH CAROLINA (2 reactors), 13

South Texas Project

Unit 1,5

Unit 2,5

Southwest Experumental Fast Oxide Reactor, 27

SP-100 Ground Engineernng System Reactor, 7

SP-100GES, 7

SP/SE, 40

SPADEFISH, 13

Spain

Almaraz

Unit 1,16

Unit 2, 16

Asco

Unit 1,16

Unt 2, 16 


\section{NETR, 44}

Neutron Radiography Faclity

NRAD, 9

NRF, 35

NEVADA, 13

Nevada, University of, 38

New Mexion, University of, 10

NEW YORK CTTY, 13

NEWPORT NEWS, 13

NF-1, 28

NIMITZ (2 reactors), 13

Nine Mile Point Nuclear Stauon

Unit 1,3

Unit 2, 3

NIST, 8

NORFOLK, 12

North Anna Power Station

Unit 1, 3

Unit 2,

North Caroluna State University

Graphte/water, 38

PULSTAR, 10

Northrop Corporate Laboratones, 36

NR-1, 13

NRAD, 9

$\mathrm{NRF}, 35$

NRX-A2, 29

NRX-A3, 29

NRX-A4/EST, 29

NRX-A5, 29

NRX-A6, 29

NTR, 9

Nuclear Effects Reactor

FRAN, 36

KUKLA, 36

Super KUKLA, 44

Nuclear Engineenng Test Reactor, 44

Nuclear Examination Reactor, 36

Nuclear Rocket Engine Reactor Experiment (NERVA)

NRX-A2, 29

NRX-A3, 29

NRX-A5, 29

NRX-A6, 29

Nuclear Rocket Reactor Engine System Test (NERVA), 29

Nuclear Rocket Reactor Experimen

Kiw1-A, 29

Kiw1-A Prime, 29

Kiw1-A3, 29

Kiw1-B1A, 29

KIW1-B1B, 29

Kıw1-B4A, 29

Pathfinder Atomic Plant, 23

PATRICK HENRY, 42

S10FS $-4,28$

S10FS-5, 28

Pawling Research Reactor, $36 \quad$ S1C, 14

PBF, 8

PCA, 22

PCTR, 36

PDP, 40

Peach Bottom Atomic Power Station

Unit 1,23

Unit 2,4

Unit 3,4

Penn State TRIGA Reactor, 10

PENNSYLVANIA, 13

PERMT, 42

Perry Nuclear Power Plant

Unit 1,4

Unit 2, 6

Pewee-1, 28

Pewee-2, 28

PHILADELPHIA, 13

Philippines

Republic of the, PRR-1, 20

Republic of the Philippine Nuclear Power

Plant, Unit 1, 17

Phoebus 1A, 30

Phoebus 1B, 30

Phoebus 2A, 30

PHOENIX, 12

PHRENIC, 34

Physical Constants Test Reactor, 36

Pilgnm Nuclear Power Station, Unit 1, 4

PINTADO, 13

Piqua Nuclear Power Faclity, 24

PITTSBURGH, 12

Planet, 22

Plum Brook Reactor Facilty, 30

PLUNGER, 42

Plutonium Recycle Test Reactor, 27

PM-1, 41

PM-2A, 41

PM-3A, 41

PNL Critical Mass Laboratory

FEAS, 47

Honzontal, 47

RSTM, 47

POGY 12

Point Beach Nuclear Plant

Unit 1, 4

Unit 2, 4

POLLACK, 42

Polytechnic Institute of New York, 39

Portable Medium Power Plant No 1, 4

No $2 \mathrm{~A}, 41$

No $3 \mathrm{~A}, 41$
$S 1 G, 43$

S1W, 43

S2DS, 27

S3G, 43

SSG, 14

S5W, 45

S8DR, 28

S8ER, 28

S8G, 14

Salem Nuclear Generatıng Station

Unit 1, 4

Unit 2, 4

SALT LAKE CTTY, 12

SAM HOUSTON, 42

SAM RAYBURN, 12

SAN FRANCISCO, 12

SAN JUAN, 13

San Onofre Nuclear Generatung Stauon

Unit 1, 4

Unit 2,5

Unit 3,5

SAND LANCE, 13

Sandia Enguneenng Reactor, 31

Sandia Pulsed Reactor, 36

Sandia Pulsed Reactor II, 9

Sandia Pulsed Reactor III, 9

SANTA FE, 13

SARGO, 42

Saxton Nuclear Experimental Reactor Project, 27

SCAMP, 42

SCHIZO, 33

SCORPION, 42

SCRANTON, 13

SCULPIN, 42

SEA DEVIL, 42

SEA HORSE, 13

Seabrook Nuclear Station, Untt 1,5

SEADRAGON, 42

SEAWOLF, 14

SEAWOLF PWR, 42

SEAWOLF Sodium Reactor, 42

SEFOR, 27

Sequoyah Nuclear Plant

Unt 1,5

Unit 2,5

SER, 27

SHARK, 42

Shearon Harris Nuclear Power Plant, Unit 1,5

SHEBA, 22

Shield Test and Irradiation Reactor, 37

Shippingport Atomic Power Station, 24
Cofrentes, Unit 1, 16

José Cabrera, 16

Lemoniz

Unt 1, 17

Unit 2, 18

Nuclear Energy Board-JEN, 20

Santa Mana de Garoña, 17

Valdecaballeros

Unit 1,18

Unit 2, 18

Vandellos, Unit 2, 17

Special Power Excursion Reactor Test

No 1,32

No 2,32

No 3,32

No 4,32

SPERT-1, 32

SPERT $-2,32$

SPERT $-3,32$

SPERT-4, 32

SPR, 36

SPR-II, 9

SPR-III, 9

SPRINGFIELD, 13

SR 305-M Test Pile, 40

SRE, 27

SS-CF, 47

SSRF, 47

St Lucie Plant

Unit 1,5

Unit 2, 5

Standard Pile/Subcrucal Experimental

Complex, 40

State University of New York, 10

Stationary Low Power Plant No 1, 43

Stationary Medium Power Plant

No 1,41

No $1 A, 41$

Stationary Neutron Radıgraphy System, 15

STIR, 37

STONEWALL JACKSON, 12

STURGEON, 12

STURGIS Floatung Nuclear Power Plant, 4

Submanne

SSN742, 13

SSN743, 13

Submanne Advanced Reactor Prototype, 43

Submarne Intermediate Reactor Mark A, 43

Submanne Thermal Reactor Faclity, 43

SUNFISH, 13

Super KUKLA, 44

SUPO, 35

Surry Power Station

Unit 1,5 


\section{REACTOR INDEX (ContInued)}

Susquehanna Steam Electnc Station

Unit 1, 5

Unit 2,

Sweden

Ringhals

Unit 2,17

Unit 3, 17

Unit 4, 17

Studsvik Energiteknık, 19

Switzerland

Paul Scherrer Institute, 20

Beznau, Unit 1, 17

Beznau, Unit 2, 17

Leibstadt, 17

Muhleberg, 17

University of Basel, 21

University of Geneva, 46

SWORDFISH, 42

Taiwan

Chinshan

Unit 1, 17

Unit 2, 17

Kuo Sheng

Unit 1,17

Unit 2, 17

Maanshan

Unit 1,17

Unit 2,17

Unit 7,18

Unit 8, 18

TAUTOG, 12

TECUMSEH, 12

TENNESSEE, 13

Test Pile, 40

TEXAS (2 reactors), 13

Texas A\&M University

AGN-201M-106, 10

TRIGA, 10

Texas at Ausun, University of

TRIGA-Mk I, 39

TRIGA-Mk II, 11
Thailand, TRR-1,20

THEODORE ROOSEVELT, 42

THEODORE ROOSEVELT (2 reactors), 13

Thermal Test Reactor No 1,44

Thermal Test Reactor No 2, 37

THOMAS A EDISON, 42

THOMAS JEFFERSON, 42

Three Mile Island Nuclear Station

Unit 1, 5

Unit 2, 24

THRESHER, 42

TINOSA, 12

TOLEDO, 14

TOPEKA, 13

Torrey Pines, TRIGA-Mk III Reactor, 37

TORY IIA, 43

TORY IIC, 43

Tower Shielding Reactor, 37

Tower Shielding Reactor No II, 9

Transient Reactor Test, 8

TREAT, 8

TREPANG, 13

Tndent Prototype, 14

TRITON (2 Reactors), 42

Trojan Nuclear Plant, Unit 1, 5

TRUXTUN (2 reactors), 13

TSR, 37

TSR-2, 9

TTR 1,44

TTR-2, 37

TUCSON, 14

TULLIBEE, 42

TUNNY, 13

Turkey

Atomic Energy Commission, 20

Technical University of Istanbul, 20

Turkey Point Plant

Unit 3,5

Unit 4,5

Tuskegee Instutute, 39

U S Geological Survey Laboratory, 9

UFTR, 9
UHTREX, 27

ULR, 10

Ultra High Temperature Reactor Experiment, 27

ULYSSES S GRANT, 12

UMR-R, 10

United Kingdom, Queen Mary College, London

University, 46

United Nuclear Corporation, Development

Division, 47

UNITED STATES, 14

Utah, University of

AGN-201-107, 11

TRIGA-Mk I, 3

UTR Test Reactor, 37

UTR-1, 33

UTR-10, 10

UVAR, 11

Vallecitos Boilng Water Reactor, 27

VBWR, 27

Venezuela, 20

Venus, 47

Vermont Yankee Nuclear Power Statuon, 5

Verucal/Split Table, 22

Vietnam, 45

Virgl C Summer Nuclear Statıon, Unit 1, 5

VIRGINIA (2 reactors), 13

Virginia, University of

CAVALIER, 39

UVAR, 11

Virginia Polytechnic Instutute, 39

VON STEUBEN, 12

Walter Reed Research Reactor, 44

Washington, University of, 39

Washington Nuclear Project

Unit 1,6

Unit 2,5

Unit 3,6

Washington State University, 11

Water Reflector Tank, 22

Waterford Generating Station, Unit 3, 5
Watts Bar Nuclear Plant

Unit 1,6

Unit 2, 6

West Berlun, City of 45

WEST VIRGINIA, 13

West Virginia University, 39

Wesunghouse Nuclear Trauning Center, 37

Wesunghouse Testung Reactor 30

WHALE 12

WILL ROGERS, 13

William B McGurre Nuclear Station

Unit 1,5

Unt 2,5

WILLIAM H BATES, 13

William Marsh Rice University, 39

Wisconsin, University of, 11

WNTR, 37

Wolf Creek Generatung Station, 6

WOODROW WILSON, 12

Worcester Polytechnic Insutute, 11

WRRR, 44

WSTR, 11

WTR, 30

Wyoming, University of, 39

XE-Backup, 28

XE-Pnme, 29

Yankee Nuclear Power Station, 6

Yugoslavia

Josef Stefan Nuclear Instutute, 20

Nukleama Elektrama Krsko, 17

Zaire, 20

Zion Nuclear Plan

Unit 1,6

Unit 2, 6

ZPPR, 22

ZPR, 9

ZPR-6, 46

ZPR-9, 47 